\title{
Solar Flares: Magnetohydrodynamic Processes
}

\author{
Kazunari Shibata \\ Kwasan and Hida Observatories, Kyoto University \\ Kyoto, 607-8471, Japan \\ email: shibata@kwasan.kyoto-u.ac.jp \\ Tetsuya Magara \\ Dept. of Astronomy and Space Science, \\ Kyung Hee University \\ Yongin, 446-701, Korea \\ email: magara@khu.ac.kr
}

Accepted on 21 September 2011

Published on 15 December 2011

\begin{abstract}
This paper outlines the current understanding of solar flares, mainly focused on magnetohydrodynamic (MHD) processes responsible for producing a flare. Observations show that flares are one of the most explosive phenomena in the atmosphere of the Sun, releasing a huge amount of energy up to about $10^{32} \mathrm{erg}$ on the timescale of hours. Flares involve the heating of plasma, mass ejection, and particle acceleration that generates high-energy particles. The key physical processes for producing a flare are: the emergence of magnetic field from the solar interior to the solar atmosphere (flux emergence), local enhancement of electric current in the corona (formation of a current sheet), and rapid dissipation of electric current (magnetic reconnection) that causes shock heating, mass ejection, and particle acceleration. The evolution toward the onset of a flare is rather quasi-static when free energy is accumulated in the form of coronal electric current (field-aligned current, more precisely), while the dissipation of coronal current proceeds rapidly, producing various dynamic events that affect lower atmospheres such as the chromosphere and photosphere. Flares manifest such rapid dissipation of coronal current, and their theoretical modeling has been developed in accordance with observations, in which numerical simulations proved to be a strong tool reproducing the time-dependent, nonlinear evolution of a flare. We review the models proposed to explain the physical mechanism of flares, giving an comprehensive explanation of the key processes mentioned above. We start with basic properties of flares, then go into the details of energy build-up, release and transport in flares where magnetic reconnection works as the central engine to produce a flare.
\end{abstract}

This review is licensed under a Creative Commons 


\section{Imprint / Terms of Use}

Living Reviews in Solar Physics is a peer reviewed open access journal published by the Max Planck Institute for Solar System Research, Max-Planck-Str. 2, 37191 Katlenburg-Lindau, Germany. ISSN 1614-4961.

This review is licensed under a Creative Commons Attribution-Non-Commercial-NoDerivs 3.0 Germany License: http://creativecommons.org/licenses/by-nc-nd/3.0/de/

Because a Living Reviews article can evolve over time, we recommend to cite the article as follows:

Kazunari Shibata and Tetsuya Magara,

"Solar Flares: Magnetohydrodynamic Processes",

Living Rev. Solar Phys., 8, (2011), 6. [Online Article]: cited [<date $>$ ],

http://www.livingreviews.org/lrsp-2011-6

The date given as $<$ date $>$ then uniquely identifies the version of the article you are referring to.

\section{Article Revisions}

Living Reviews supports two ways of keeping its articles up-to-date:

Fast-track revision A fast-track revision provides the author with the opportunity to add short notices of current research results, trends and developments, or important publications to the article. A fast-track revision is refereed by the responsible subject editor. If an article has undergone a fast-track revision, a summary of changes will be listed here.

Major update A major update will include substantial changes and additions and is subject to full external refereeing. It is published with a new publication number.

For detailed documentation of an article's evolution, please refer to the history document of the article's online version at http: //www.livingreviews.org/lrsp-2011-6. 


\section{Contents}

1 Introduction $\quad 5$

2 Observational Features and Phenomenological Models 12

2.1 Long-durational-event (LDE) flares . . . . . . . . . . . . . . . . . . . . 12

2.2 Giant-arcade formation associated with filament eruption (or coronal mass ejection) 14

2.3 Impulsive flares . . . . . . . . . . . . . . . . . . . . . . . . . 15

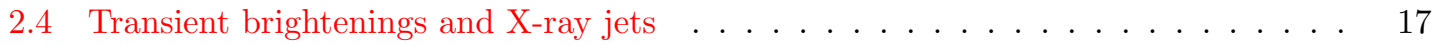

2.5 Unified model for flares, microflares, and jets . . . . . . . . . . . . . . . . . . 20

2.6 Avalanche model and non-reconnection models . . . . . . . . . . . . . . . 20

3 Energy Build-up $\quad 23$

3.1 Emergence of magnetic field (flux emergence) . . . . . . . . . . . . . . . . . . 23

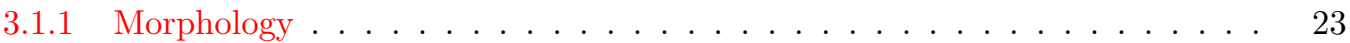

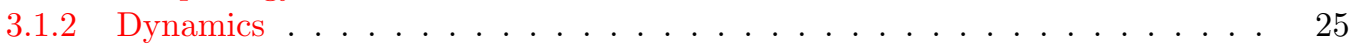

3.1 .3 Latest progress . . . . . . . . . . . . . . . . . . . . . . . . . . . . . . . . . . . .

3.2 Magnetic structure . . . . . . . . . . . . . . . . . . . 30

3.2.1 Filament (Prominence) . . . . . . . . . . . . . . . . 30

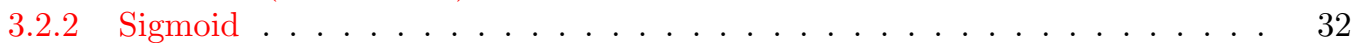

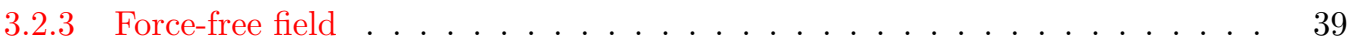

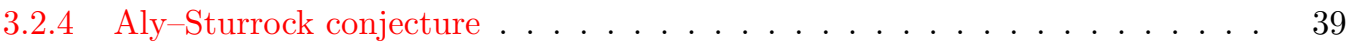

3.3 Magnetic helicity . . . . . . . . . . . . . . . . . . . . 40

4 Energy Release $\quad \mathbf{4 5}$

4.1 Magnetic reconnection . . . . . . . . . . . . . . . . . . . . . 45

4.1 .1 Basic models of magnetic reconnection . . . . . . . . . . . . . . . 45

4.1 .2 Sweet-Parker model . . . . . . . . . . . . . . . . . . . . . . . . . . . . . . . . .

4.1 .3 Petschek model . . . . . . . . . . . . . . . . . . . . 46

4.1 .4 Locally enhanced resistivity . . . . . . . . . . . . . . . . . . . . . . . . . . . . . . . .

4.1.5 Tearing instability and fractal reconnection . . . . . . . . . . . . . . . . . 47

4.1 .6 Plasmoid-induced reconnection . . . . . . . . . . . . . . . . . 49

4.2 Current-sheet formation . . . . . . . . . . . . . . . . . . . . . . 49

4.2.1 Interaction of flux domains . . . . . . . . . . . . . . . . . . 50

4.2.2 Development of magnetic shear in single flux domain . . . . . . . . . . . . . 55

4.3 Modeling of energy-release processes . . . . . . . . . . . . . . . . . . . 57

4.3.1 Bipolar system (single flux domain) . . . . . . . . . . . . . . . 57

4.3.2 Multi-polar system (two-step reconnection) . . . . . . . . . . . . 59

5 Energy Transport $\quad 61$

5.1 Radiation ............................ . . . 6 61

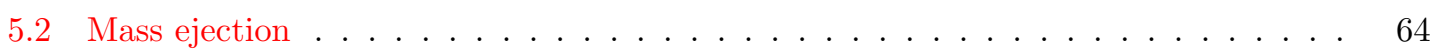

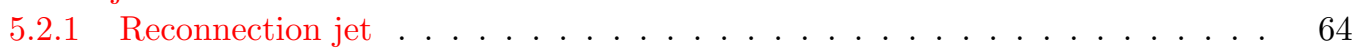

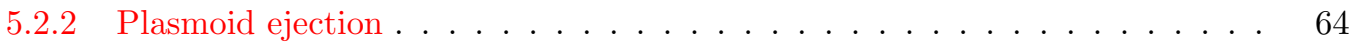

5.3 Shock formation and heating . . . . . . . . . . . . . . . . 66

5.3 .1 Slow shock . . . . . . . . . . . . . . . . . . . 66

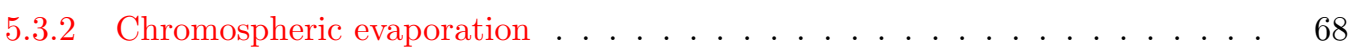

5.3 .3 Fast shock . . . . . . . . . . . . . . . . . . . . 69

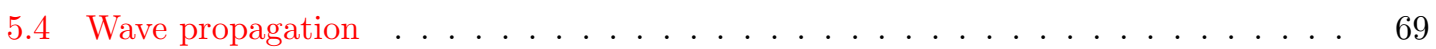

5.5 Particle acceleration ........................ . . 70 
6 Application to Stellar Flares $r$

7 Concluding Remarks $r 3$

8 Acknowledgements $r$

$\begin{array}{lr}\text { References } & \mathbf{7 6}\end{array}$

\section{List of Tables}

1 Characteristics of flares and flare-like phenomena. . . . . . . . . . . . . . . . . . 21

2 Comparison of scales and associated mass ejection. . . . . . . . . . . . . . . 22

3 Comparison of physical quantities. . . . . . . . . . . . . . . . . . . . . 22

4 Unified view of flares and flare-like phenomena. . . . . . . . . . . . . . . . . . 22 


\section{Introduction}

Solar flares are explosive phenomena observed in the solar atmosphere filled with magnetized plasma. The energy released during a flare is about $10^{28}-10^{32} \mathrm{erg}$, and it takes various forms such as radiative energy, kinetic bulk energy, thermal and nonthermal energy. Because of their magnificent behavior, flares have been one of the most attractive scientific targets of the solar physics since they were first observed in the 19th century. The spatial size of a flare depends on individual events; in the smallest event the height of a flaring loop is less than $10^{4} \mathrm{~km}$, whereas it reaches $10^{5} \mathrm{~km}$ in the largest event (see Section 2). The size also affects the duration of a flare $\left(10^{3}-10^{4} \mathrm{~s}\right)$ and the amount of energy released during a flare mentioned above.

Flares are observed in a wide range of electromagnetic waves such as radio, visible light, Xrays, and gamma rays (see Section 5). Emissions in these wavelengths come from the atmospheric layers extending from the chromosphere to the corona. In the extreme case, even the photosphere responds to a big flare, observed as white-light brightenings. Also a flare produces high-energy particles which travel through the interplanetary space, sometimes having a severe impact on the environment of the Earth (see Section 7).

Historically, flares were discovered in white light (Carrington, 1859; Hodgson, 1859). Later, spectroheliographs developed and an $\mathrm{H} \alpha$ filter was invented, flares can be observed in $\mathrm{H} \alpha$. An $\mathrm{H} \alpha$ monochromatic image of a flare often shows beautiful two ribbons of bright patches, and the distance between these ribbons increases with time (e.g., Švestka, 1976; Zirin, 1988). For a long time flares were considered as chromospheric phenomena observed in $\mathrm{H} \alpha$. However, the discovery of coronal radio and X-ray emissions from a flaring site has revealed that flares are actually coronal phenomena.

Since the discovery of magnetic field on the Sun (Hale, 1908), the role of magnetic field in solar activity has been investigated extensively. Skylab mission (1973-1974) first made a detailed survey of the corona from the space using a soft X-ray telescope, revealing that bright regions in soft X-ray are well correlated with intense magnetic field. It is now widely accepted that the magnetic field provides a main energy source of the solar activity including flares. Observations suggest that the total magnetic energy stored in a typical sunspot with the size $L$ and the averaged magnetic field $B$ is

$$
E_{\mathrm{mag}} \simeq\left(B^{2} / 8 \pi\right) L^{3} \simeq 10^{33}\left(\frac{B}{10^{3} \mathrm{G}}\right)^{2}\left(\frac{L}{3 \times 10^{9} \mathrm{~cm}}\right)^{3} \mathrm{erg},
$$

which is sufficient to produce even the largest flare, although only a small portion of this total energy can be used, that is, a large amount of energy is unavailable because it is distributed as the potential field energy.

Following observational results, theoretical studies began to focus on the role of magnetic field in producing a flare. Giovanelli (1946) first pointed out that a neutral point where the magnetic field takes an X-type configuration could be the site of energy release during a flare. He proposed that the electric current may be dissipated at the neutral point. Hoyle (1949) also presented a similar idea about the mechanism for producing a flare, which is now known as magnetic reconnection. As Cowling (1953) later pointed out, however, there was difficulty in explaining the time scale of a flare if we assume that this is given by simple diffusion of magnetic field (no contribution of plasma flow). This is because the time scale of diffusion is much longer than the typical time scale of a flare in the corona where both the temperature of plasma $(T)$ and the length scale of magnetic field $(L)$ take a large value. The diffusion time in the corona is given by

$$
t_{\text {dif }} \simeq L^{2} / \eta \simeq 10^{14}\left(L / 10^{9} \mathrm{~cm}\right)^{2}\left(T / 10^{6} \mathrm{~K}\right)^{3 / 2} \mathrm{~s},
$$

where

$$
\eta \simeq 10^{4}\left(\frac{T}{10^{6} \mathrm{~K}}\right)^{-3 / 2} \mathrm{~cm}^{2} \mathrm{~s}^{-1}
$$




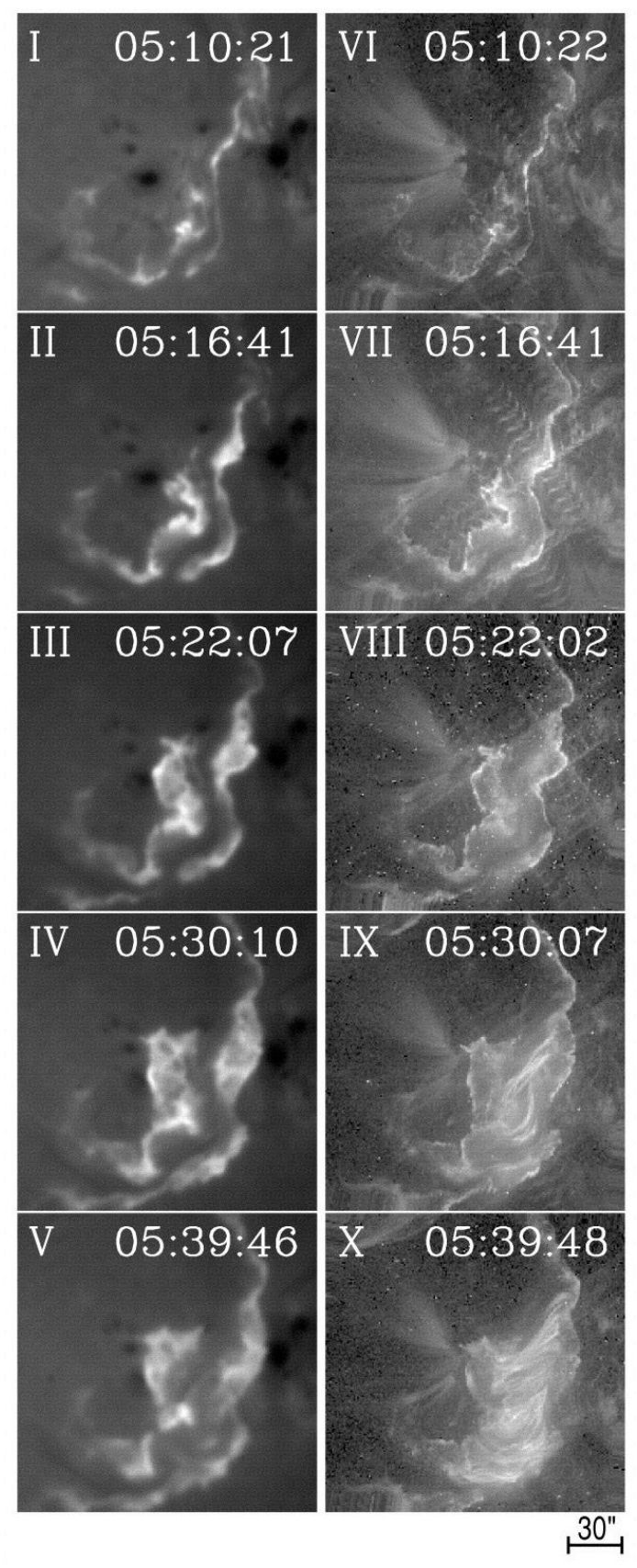

Figure 1: Two ribbons of a flare observed in $\mathrm{H} \alpha(\mathrm{I}-\mathrm{V})$ and $\mathrm{EUV}(\mathrm{VI}-\mathrm{X})$. H $\alpha$ data are taken at Kwasan Observatory of Kyoto University, and EUV data are taken with EUV telescope aboard TRACE (from Asai et al., 2003). 
is the magnetic diffusivity derived from the Spitzer resistivity (Spitzer, 1962). In order to overcome this big gap of these two time scales, the spatial scale of a diffusion region where electric current is dissipated must be as small as $L \sim 10^{3} \mathrm{~cm}$, although the total energy released during a flare cannot be explained by such a small diffusion region. Later, a model of magnetic reconnection that takes plasma flow into account was proposed by Sweet (1958) and Parker (1957). However, this reconnection model, which is now known as the Sweet-Parker reconnection, still cannot explain the time scale of a flare (Parker, 1963). This problem was partially solved by Petschek (1964)'s remarkable idea in which slow magnetohydrodynamic (MHD) standing shock waves are introduced to the reconnection dynamics (Petschek model, see Section 4). Since then, the magnetic reconnection has been considered to be one of the promising mechanisms for producing a flare, although a complete understanding of the relevant physics is still on the way.

a)

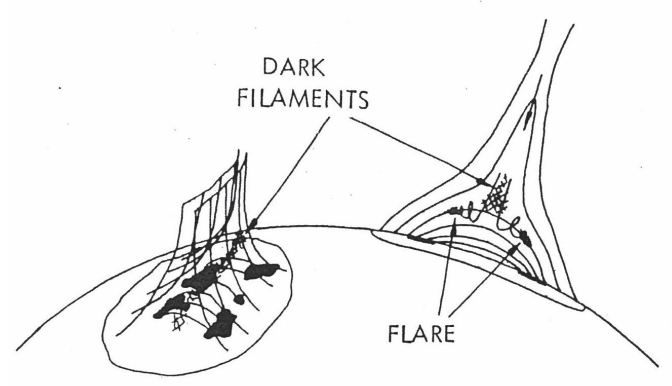

c)
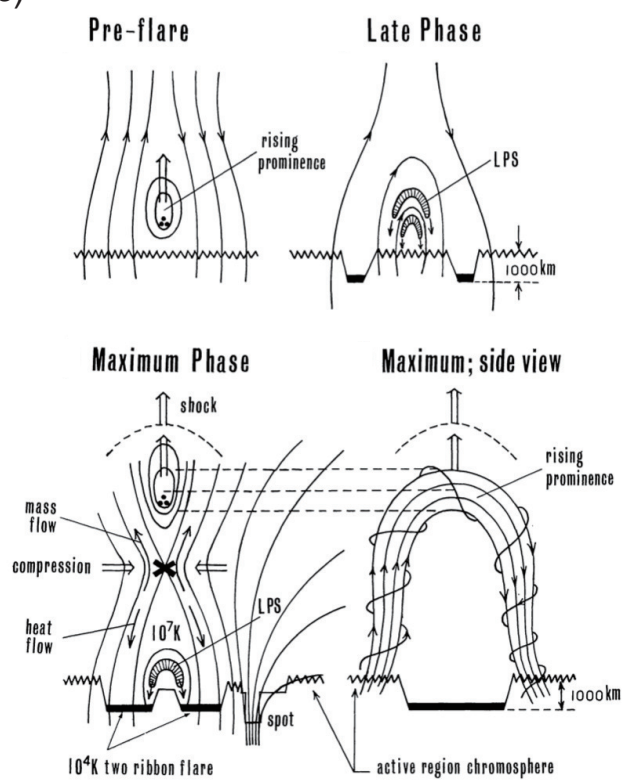

b)
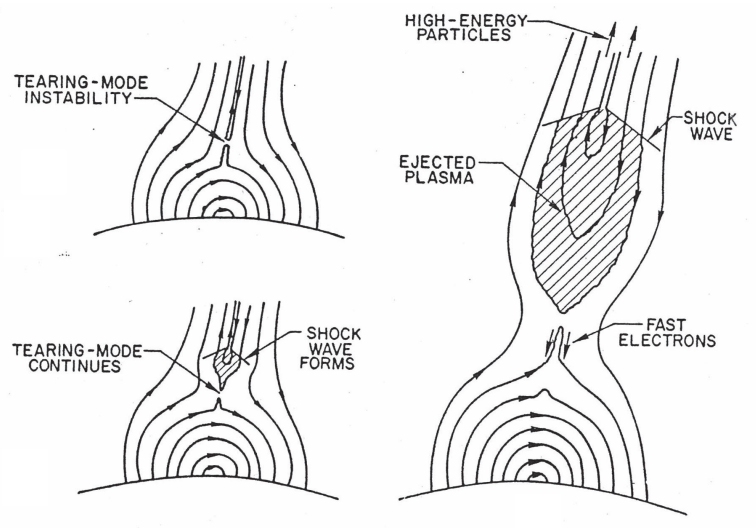

d)
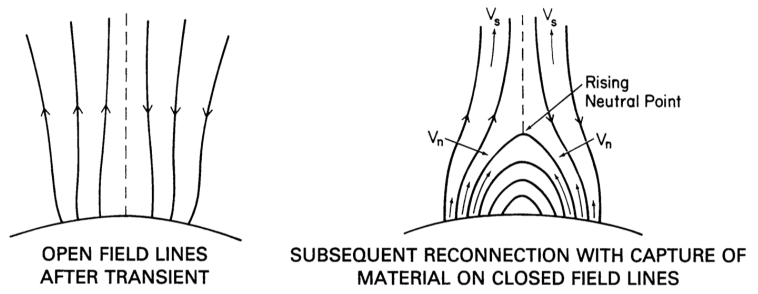

Figure 2: Phenomenological models for flares based on magnetic reconnection. Lines with arrows on them indicate magnetic field lines. (a) Carmichael (1964), (b) Sturrock (1966), (c) Hirayama (1974), (d) Kopp and Pneuman (1976). 
Several classic models based on magnetic reconnection have been proposed to explain the phenomenological aspect of flares: Carmichael (1964), Sturrock (1966), Hirayama (1974), and Kopp and Pneuman (1976) (see Figure 2). These models assume more or less a similar configuration of magnetic field and its dynamic process, so these models are called with a single name, CSHKP model (Švestka and Cliver, 1992; Sturrock, 1992; Shibata, 1999). The CSHKP model has been a standard model of flares, and the basic features of this model are explained in Figure 3. We also show a recent observational image obtained by Yohkoh in this figure.

Here we show a brief history about the naming of "CSHKP". In the 1980s, this model was called the "Kopp-Pneuman model" in the United States. Shibata (1991) proposed to change the name from Kopp-Pneuman model to "SHKP model", by respecting the pioneering works by Sturrock and Hirayama. Soon after, Sturrock himself proposed to add "C" in front of "SHKP" in his 1992 proceedings (Sturrock, 1992), noting another pioneering work by Carmichael. Švestka and Cliver (1992) also use the term "CSHKP model" in the same proceedings book.

The CSHKP model has been improved significantly since Kopp and Pneuman (1976), especially because the theory of magnetic reconnection has been developed, which is summarized in a nice review paper by Priest and Forbes (2002). Kopp and Pneuman (1976) considered that open field lines reconnect to form a closed loop, then the upflows of the solar wind along the reconnected field lines collide each other to generate a shock inside the closed loop, thereby heating coronal plasma up to the typical temperature of a flare. However, Cargill and Priest (1982) later pointed out that it is important to consider the slow MHD shocks suggested by Petschek (1964). Forbes and Priest (1984) noted that a fast MHD shock (termination shock) is also formed above the reconnected loop when a reconnection jet collides with this loop. Furthermore, Forbes and Malherbe (1986) showed that the slow MHD shock could be dissociated to an isothermal slow shock and a conduction front in the case of flares. Those features mentioned above have been reproduced by recent MHD simulations (Yokoyama and Shibata, 1997, 1998, 2001), which are discussed in Section 5.

As we mentioned, the theory of magnetic reconnection is not completed yet, not only in solar plasma but also in laboratory and magnetospheric plasma. This is why many anti-reconnection models of a flare have been proposed (e.g., Alfvén and Carlqvist, 1967; Akasofu, 1984; Melrose, 1997; Uchida and Shibata, 1988, etc.). However, recent space missions such as Yohkoh (19912001), SOHO (SOlar Heliospheric Observatory; 1995-), TRACE (Transition Region and Coronal Explorer; 1998-), RHESSI (Ramaty High Energy Solar Spectroscopic Imager; 2002-), and Hinode (2006-) have provided a huge amount of observational results suggesting that the magnetic reconnection occurs during a flare (e.g., Shibata, 1999). These observational results supporting the reconnection model of a flare are summarized in Section 3.

The magnetic field providing the energy source of a flare originally comes below the solar surface (Zwaan, 1985), after traveling across the convection zone. While it travels across this region, the magnetic field is surrounded by a high-pressure plasma doing convective motions (e.g., Parker, 1979), so the magnetic field may take the form of a thin flux tube with some twist (Fan, 2009). When such a flux tube emerges into the surface, the background gas pressure decreases abruptly, so the magnetic field expand rapidly. The emerging magnetic field eventually fills a large volume up in the solar atmosphere and forms a magnetic structure there. The magnetic field loses the magnetic energy originally stored in the flux tube through this process, although part of magnetic energy remains in the magnetic structure as field-aligned electric current which does not produce the Lorentz force. The field-aligned current is therefore stored as free energy unless it is dissipated. The field-aligned current also introduces distortion into the magnetic structure (Sakurai, 1979), which is observed as a sheared arcade and/or twisted flux rope. The formation of distorted magnetic structure is important for understanding the state that leads to the onset of a flare, and we discuss this topic in Section 3.

It has been known that there are events preceding the onset of a flare. These are called 'precursors', and one of the prominent precursors is a newly emerging bipolar region at the surface,

Living Reviews in Solar Physics

http://www. livingreviews.org/lrsp-2011-6 


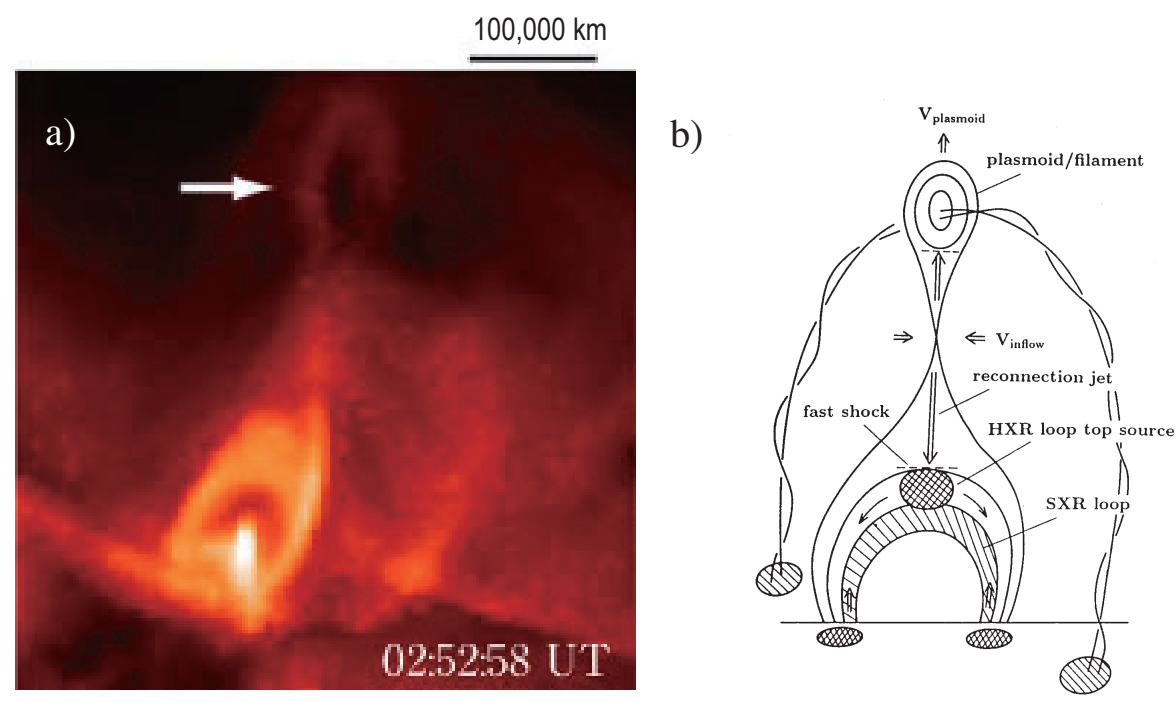

Figure 3: (a) Soft X-ray image of a long-durational-event ( $L D E$ ) flare (see Section 2) observed by Yohkoh. (b) Schematic picture of a modified version of the CSHKP model, incorporating the new features discovered by Yohkoh (from Shibata et al., 1995).

which may interact with preexisting magnetic field in the corona and produce a flare (Rust, 1968; Martres et al., 1968; Zirin, 1974; Heyvaerts et al., 1977; Martin et al., 1982; Feynman and Martin, 1995). Another well-known precursor is the activation, or eruption of a filament. A filament is composed of a relatively cool plasma with $T \sim 10^{4} \mathrm{~K}$, floated in the corona that is occupied by a much hotter plasma with $T \geq 10^{6} \mathrm{~K}$. The activation of a filament suggests that some destabilization proceeds in a magnetic structure containing a filament. Since a filament is formed in a low-beta coronal region (magnetic pressure is dominant compared to gas pressure), the main forces causing such destabilization are the gradient force of magnetic pressure, magnetic tension force, and gravitational force. It is therefore important to know how these three forces keep balance during the pre-eruptive phase of a filament, and under what condition the balance is lost to cause the eruption of a filament. This issue is related to the onset of a flare and discussed in (Section 3).

Since the corona is filled with a highly conductive medium, dissipating electric current there is usually inefficient. This, on the other hand, indicates that the magnetic energy tends to be stored in the corona without any easy, continuous dissipation of electric current. An important question then arises regarding how to release the magnetic energy in the corona (otherwise tremendous amount of magnetic energy would be accumulated in the corona). One of the possible scenarios is that the magnetic energy is released via the dissipation of electric current in a thin, sheet-like region where the current density is enhanced, which is called current sheet. This is accompanied by the reconnection of magnetic field that converts the magnetic energy to not only thermal but also kinetic energy of plasma jets (high-energy particles are produced as well; selected particles are accelerated by electromagnetic force). The magnetic reconnection changes the configuration of magnetic field (field line topology) in a magnetic structure, destroying force balance to drive a global evolution of the structure. It has been suggested that a number of current sheets are spontaneously formed in the corona (Parker, 1994; Longcope, 2005; Low, 2006). Magnetic reconnection is a process of converting energies and changing topologies, so the coronal field can relax via successive reconnections to a lower energy state, avoiding a monotonous increase of the magnetic energy in the corona. We discuss the formation of a current sheet followed by rapid energy release by magnetic reconnection in Section 4. 
The energy released by magnetic reconnection is immediately transported from the site of reconnection via radiation, thermal conduction, high-energy particles, and plasma blobs (Priest and Forbes, 2002). The configuration of magnetic field significantly influences this energy transport. Part of the released energy is transported downward along magnetic field lines via thermal conduc-

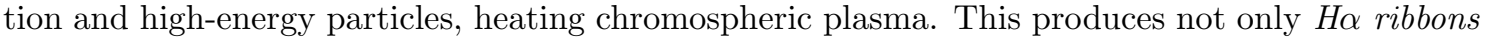
shown in Figure 1, but also hard X-ray (HXR) kernels in some energetic flares. The gas pressure of the heated chromospheric plasma increases, which drives the upflow of a plasma into the corona against gravitational force (chromospheric evaporation), filling a magnetic loop with hot plasma. This loop is observed in soft X-ray, so it is called a soft X-ray (SXR) loop. Another part of the released energy is transported upward as an ejecting plasma blob called plasmoid. During this phase selected particles are accelerated to become nonthermal energetic particles (Ramaty and Murphy, 1987). Those processes mentioned above are highly dynamic and complex, so numerical simulations are quite useful and indispensable for a better modeling of time-dependent, nonlinear energy transporting processes. In Section 5, we show the result obtained from such numerical simulations and explain key issues of energy transport during a flare.

A flare is often associated with other dynamic phenomena on the Sun. As we mentioned, the activation of a filament is one of those flare-associated phenomena. Sometimes a flare is part of a large-scale eruption known as a coronal mass ejection (CME), which carries a tremendous amount of plasma (up to $10^{16} \mathrm{~g}$ ) to the interplanetary space. In a typical CME, a large magnetic loop with the size of the solar radius moves away from the Sun at $30-2500 \mathrm{~km} \mathrm{~s}^{-1}$ (Yashiro et al., 2004), which forms a shock wave at the front of the erupting loop. Regarding the modeling of CME, there are several concise and comprehensive reviews (Forbes, 2000; Klimchuk, 2001; Gibson et al., 2006; Mikić and Lee, 2006; Forbes et al., 2006; Chen, 2011). The physical relationship between a flare and CME has intensively been investigated (Gosling, 1997).

It should be noted that this paper is mainly focused on the MHD aspects of a flare. Hence, for readers who are interested in other aspects of a flare such as nonthermal processes of particles we introduce the following papers and books: Švestka (1976), Priest (1981), Dulk (1985), Dennis (1985), Sakai and Ohsawa (1987), Tandberg-Hanssen and Emslie (1988), Haisch et al. (1991), Kahler (1992), Hudson and Ryan (1995), Sakai and de Jager (1996), Low (1996), Low (2001), Shibata (1997), Shibata (1999), Miller (1997), Bastian et al. (1998), Hundhausen (1999), Forbes (2000), Charbonneau et al. (2001), Aschwanden (2002), Priest and Forbes (2002), Zhang and Low (2003), Lin et al. (2003). The readers can also find a well-described explanation of solar flares in the following books: Parker (1979), Parker (1994), Melrose (1980), Priest (1982), Zirin (1988), Stix (2004), Benz (1993), Tajima and Shibata (1997), Golub and Pasachoff (1997), Somov (2000), Priest and Forbes (2000), Aschwanden (2004). Let us show the organization of this paper. In Section 2, we summarize observational features of a flare and flare-like phenomena, and show their phenomenological models. Section 3 describes energy build-up via flux emergence which plays the central role in forming a sheared magnetic structure with the free energy stored inside it. In this section we discuss the dynamics of an emerging flux tube to derive the basic feature of a magnetic structure evolving into the onset of a flare such as a filament and sigmoid. In this respect, force-free modeling and magnetic helicity are also discussed. Section 4 is focused on magnetic reconnection, the central engine that converts free energy to produce various dynamic processes. We start with the basic concept of magnetic reconnection and then demonstrate its application in the physics of flares. In Section 5, we show various processes associated with energy transport in a flare, such as radiation, mass ejection, shock heating, wave propagation, and particle acceleration. In Section 6, stellar flares are briefly explained. Finally, we make some concluding remarks on the physical processes responsible for producing a flare. The contents of the paper are listed below.

Living Reviews in Solar Physics

http://www. livingreviews.org/lrsp-2011-6 


\begin{tabular}{lll}
\hline Section & Subject & Topics \\
\hline Section 2 & $\begin{array}{l}\text { Observational } \\
\text { features and phe- } \\
\text { nomenological } \\
\text { models }\end{array}$ & $\begin{array}{l}\text { LDE flares, Impulsive flares, Giant arcade, Transient } \\
\text { brightenings, X-ray jets, and models focused on their } \\
\text { phenomenological aspects }\end{array}$ \\
Section 3 & Energy build-up & $\begin{array}{l}\text { Flux emergence, Force-free fields, Preflare structure, } \\
\text { Magnetic helicity }\end{array}$ \\
Section 4 & Energy release & $\begin{array}{l}\text { Magnetic reconnection, Current-sheet formation, } \\
\text { Flux-rope eruption }\end{array}$ \\
Section 5 & Energy transport & $\begin{array}{l}\text { Radiation, Mass ejection, Shock heating, Particle ac- } \\
\text { celeration, Wave propagation, Chromospheric evap- } \\
\text { oration }\end{array}$ \\
Section 6 & Stellar flares & Application of solar flare model to stellar flares \\
Section 7 & Conclusions & Summary of physical processes for producing a flare \\
\hline
\end{tabular}




\section{Observational Features and Phenomenological Models}

\subsection{Long-durational-event (LDE) flares}

One of the biggest discoveries by the soft X-ray telescope (SXT) aboard Yohkoh is the cusp-shaped loop structure of long-durational-event (LDE) flares. Figure 4 shows a beautiful example of an LDE flare, which was observed on Feb. 21, 1992 at the west limb of the Sun (Tsuneta et al., 1992a; Tsuneta, 1996; Forbes and Acton, 1996). This flare is accompanied by a large-scale coronal ejection (possibly coronal mass ejection). The ejection created a helmet streamer-like configuration, suggesting that a current sheet (Section 4.1) is formed. The apparent height of the cusp-shaped loop and the distance between the footpoints of the loop increased gradually at a few $\mathrm{km} \mathrm{s}^{-1}$. This supports the idea that magnetic reconnection (Section 4.1) successively occurred above the loop.

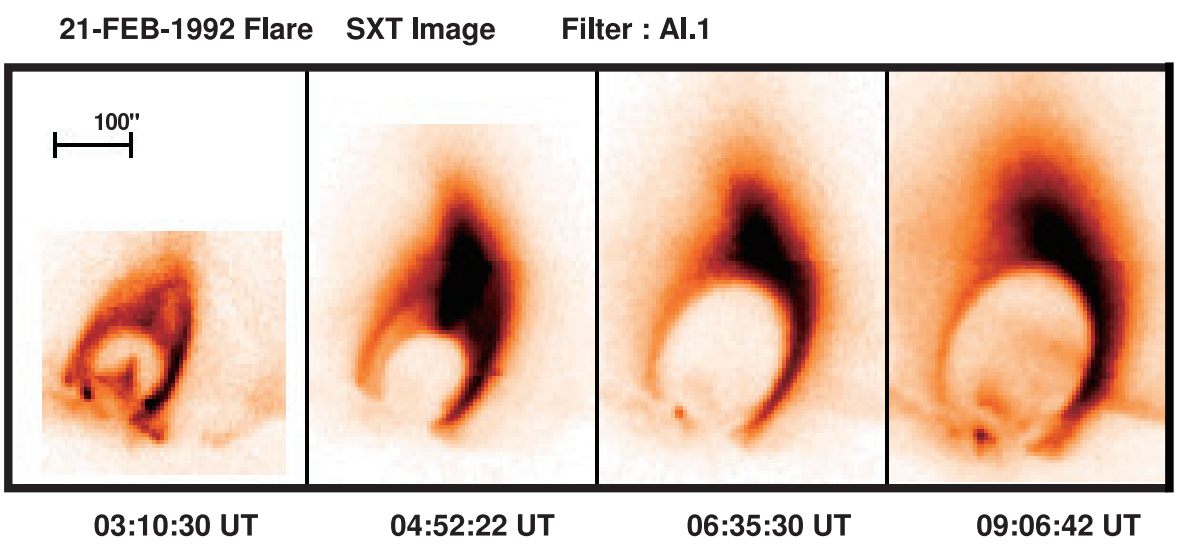

Figure 4: A soft X-ray image of an LDE flare with cusp shaped-loop structure, observed on Feb. 21, 1992 (Tsuneta et al., 1992a; Tsuneta, 1996). Shown in reversed contrast.

Tsuneta et al. (1992a) derived the following features of this LDE flare. These are now recognized as the common properties of LDE flares: The temperature distribution is somewhat chaotic in the early phase when the flare started, while in the late phase the temperature is systematically higher near the edge of the cusp-shaped loop (Veronig et al., 2006, and references therein). This can be explained by the radiative cooling efficiently working at inner central part of the loop (Forbes and Malherbe, 1991; Vršnak et al., 2006).

Yohkoh observations of the same flare as in Figure 4 (Hudson, 1994) showed that a small island-like feature (plasmoid) with the size of a few $10^{4} \mathrm{~km}$ in soft X-ray is ejected at about a few $100 \mathrm{~km} \mathrm{~s}^{-1}$ during the precursor phase (see Figure 3a, Figure 43) of the flare. A soft X-ray movie of this flare suggests that the ejection of the plasmoid triggers the flare. It also shows that a filamentary structure existed before the onset of the flare and after it was ejected, the flare occurred. The filamentary structure seems to be located inside a current sheet which is formed during the preflare phase of this flare. It is expected that the structure formed inside a current sheet tends to inhibit strong inflows from entering a current sheet, and after this structure is ejected from the current sheet, strong inflows can drag magnetic flux into the current sheet and the rapid energy release starts (Section 4.1.6). A similar ejection phenomenon is also found in another flare (see Figure 5).

The cusp-shaped loop is also observed in many other flares (e.g., Tsuneta, 1997), even in somewhat indistinct observations done during the pre- Yohkoh era (MacCombie and Rust, 1979; Hanaoka et al., 1986).

Living Reviews in Solar Physics

http://www . livingreviews . org/lrsp-2011-6 


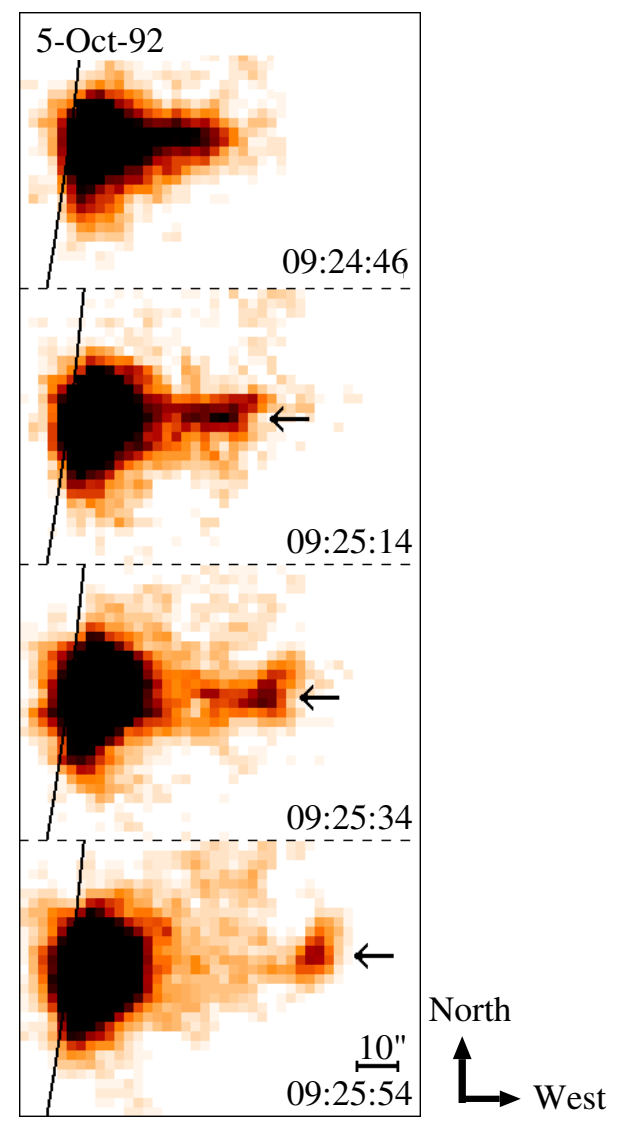

Figure 5: Soft X-ray images of plasmoid ejection associated with the flare on Oct. 5, 1992 (from Ohyama and Shibata, 1998). Shown in reversed contrast. 


\subsection{Giant-arcade formation associated with filament eruption (or coro- nal mass ejection)}

It has been found that a cusp-shaped loop or arcade is formed in the events that have much larger spatial scale than LDE flares (Tsuneta et al., 1992b; McAllister et al., 1992; Watanabe et al., 1992; Hanaoka et al., 1994; Shiota et al., 2005). These are called giant arcades, which are usually associated with the disappearance of a dark filament. Tsuneta et al. (1992b) analyzed a giant arcade associated with the disappearance of a polar-crown filament observed on Nov. 12, 1991. The arcade has gradually evolved for more than 20 hours and formed a structure with the size of more than solar radius. A similar event was also observed on Apr. 14, 1994 (McAllister et al., 1996; Figure 6).

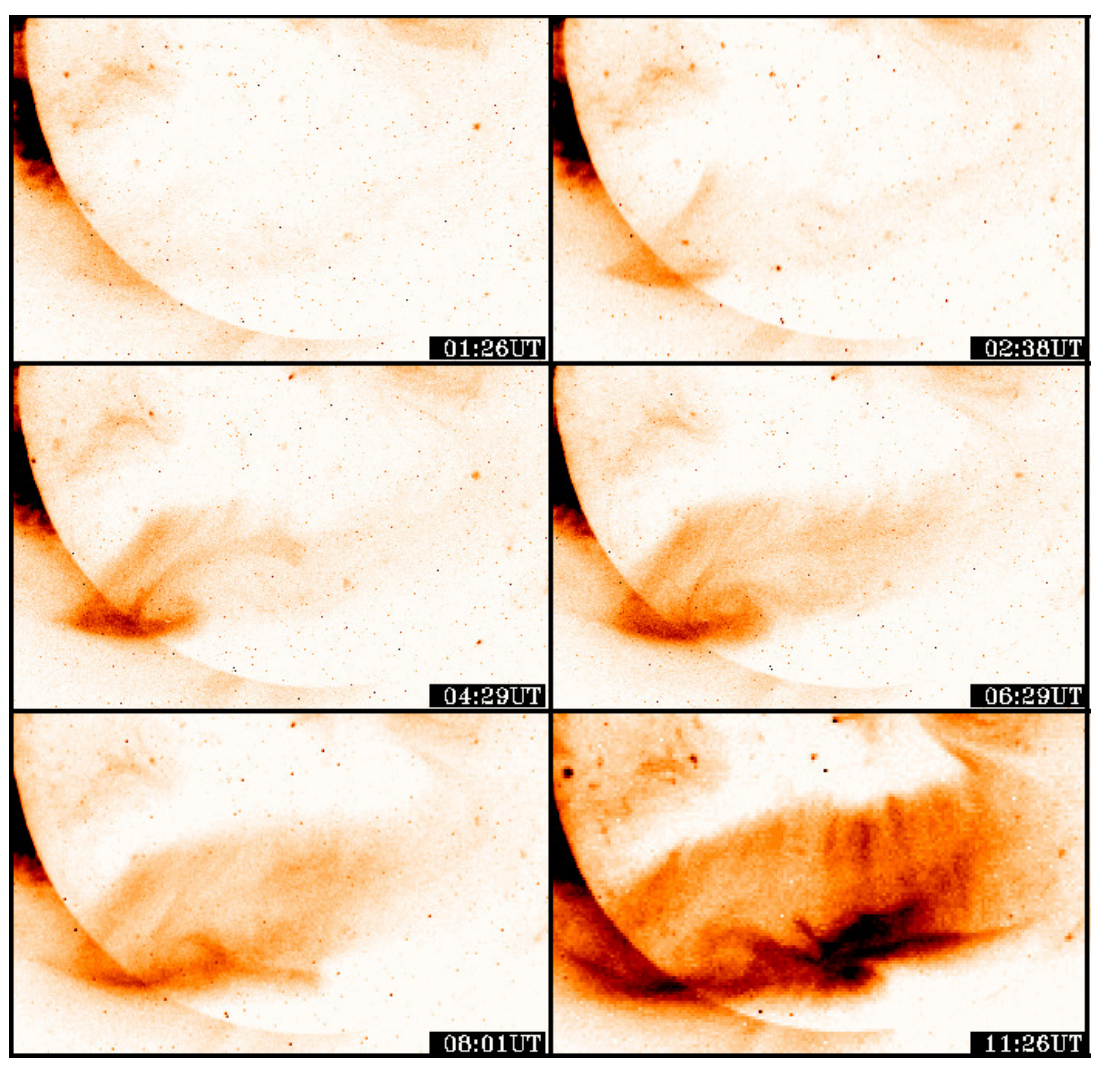

Figure 6: A giant arcade observed in soft X-ray on April 14, 1994 (from McAllister et al., 1996). Shown in reversed contrast.

A large helmet streamer is usually observed after the eruption of a filament or CME, and this probably shows the side view of a giant arcade. An example of this is presented in Figure 7 (Hiei et al., 1993). It is interesting to note that the temperature is higher near the edge of the cusp-shaped loop of giant arcades, which is similar to LDE flares. Note also that the soft X-ray intensity in a giant arcade is usually very low, and this is why giant arcades had belonged to a different category than flares. However, Yohkoh/SXT has revealed that giant arcades have a lot of similarities to LDE flares except for their spatial scale and magnetic field strength. These two factors actually affect the time scale, amount of released energy, and emission measure of individual events.

Outside the cusp-shaped structure the soft X-ray intensity often decreases with time, which is 


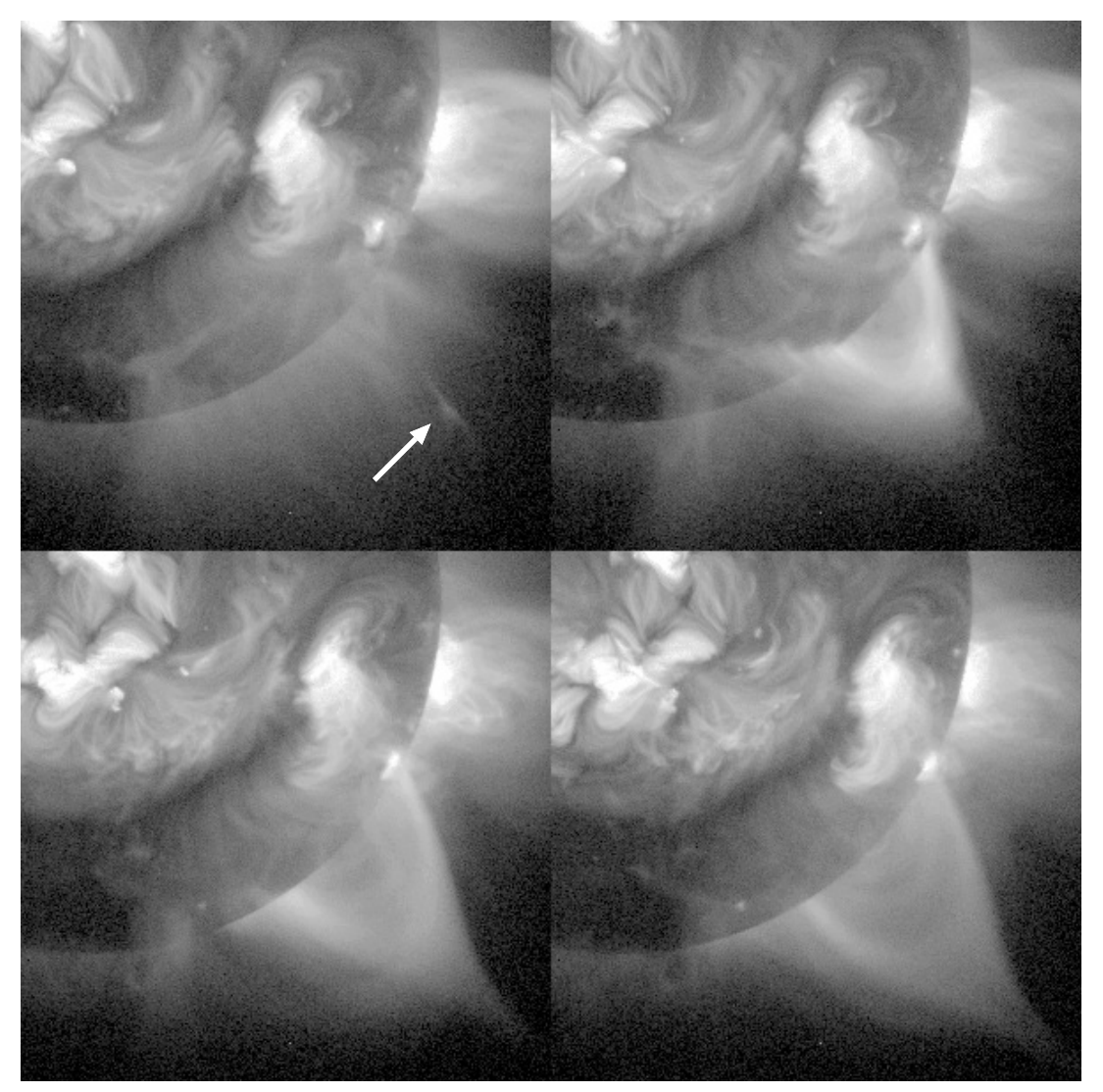

Figure 7: Helmet streamer observed in soft X-ray on January 24, 1992 (modified from Hiei et al., 1993).

called dimming (Tsuneta, 1996; Sterling and Hudson, 1997; Harra and Sterling, 2001). Tsuneta (1996) attributed dimming to the inflow driven by reconnection that carries a large amount of plasma surrounding a current sheet into the sheet, thereby decreasing the gas density outside the current sheet (see also Shiota et al., 2005). Although usual dimmings associated with CMEs are attributed to stretching /opening of field lines by eruption (e.g., Sterling and Hudson, 1997; Harra and Sterling, 2001), there is a possibility that some of them are caused by the evacuation via the inflow into reconnection region like above (e.g., Shiota et al., 2005).

\subsection{Impulsive flares}

Those flares called impulsive flares show simple loop structure in soft X-ray, and they do not have a cusp-shaped structure, as found by Skylab before Yohkoh. Because of their apparent shape, impulsive flares are also called compact flares or confined flares (Pallavicini et al., 1977). Historically, it was considered that these flares were produced via energy release inside the loop observed in soft X-ray (Alfvén and Carlqvist, 1967; Spicer, 1977; Uchida and Shibata, 1988). Obviously, this is different from the energy release outside the loop, suggested by the CSHKP model for LDE flares, in which the energy release site is a current sheet formed above the soft X-ray (SXR) loop (see Figure 42).

Using the hard X-ray telescope (HXT) aboard Yohkoh, Masuda et al. (1994, 1996) discovered a hard X-ray loop-top source above a soft X-ray loop in several impulsive flares. This suggests that the energy source producing the hard X-ray loop-top source is not located inside the soft 


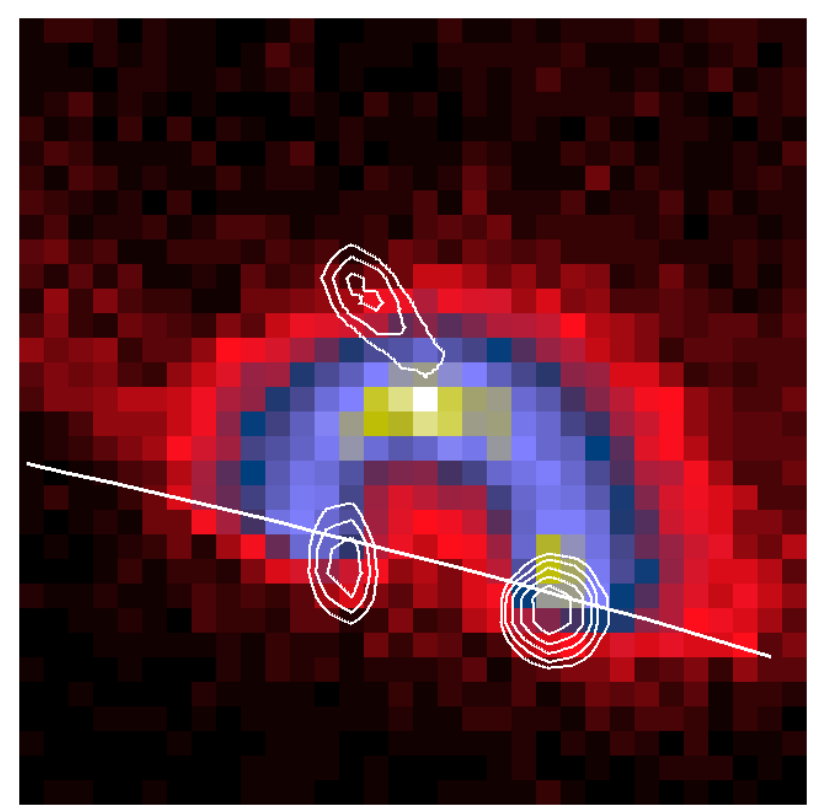

Figure 8: Hard X-ray loop-top source (contours) of an impulsive flare observed on January 13, 1992 (from Masuda, 1994). Colors represent soft X-ray intensity.

X-ray loop but above (outside) the loop. Figure 8 shows an example of hard X-ray loop-top and footpoint sources, observed at the limb of the Sun. The temporal behavior of the loop-top source is similar to the footpoint sources (Masuda, 1994). This suggests that at least part of impulsive flares are produced through the same process as LDE flares. A more detailed explanation is given in Section 4 (see also Figure 37).

Later, Shibata et al. (1995) show an evidence that magnetic reconnection occurs outside a soft X-ray loop in several impulsive flares, where they also found plasmoid ejection (see Figure 5). Shibata et al. (1995) derived the following features of plasmoid ejection observed in impulsive flares:

1. The velocity is $50-400 \mathrm{~km} \mathrm{~s}^{-1}$.

2. The size is $4-10 \times 10^{4} \mathrm{~km}$.

3. The soft X-ray intensity is $10^{-4}-10^{-2}$ of the peak intensity of a soft X-ray loop.

4. The ejection of a plasmoid starts almost simultaneously at the beginning of the impulsive phase during which hard X-ray intensity takes a peak value. This relation also holds true in the case of multiple ejection where multiple impulsive phases exist (e.g., Oct. 4, 1992 flare).

5. A small soft X-ray bright point appears during the impulsive phase of a flare (Shibata et al., 1995), about a few $10^{4} \mathrm{~km}$ away from a soft X-ray loop. It is suggested that this bright point corresponds to one of the footpoints of an erupting flux rope forming a plasmoid in three-dimensonal space.

Recently, Shimizu et al. (2008) made an analysis of fifteen impulsive flares to examine the correlation among the rise velocity of a soft X-ray loop and the ejection velocity of a plasmoid. The main conclusion is that there is a positive correlation between these two velocities (the ejection velocity is an order larger than the rise velocity), suggesting that the CSHKP model can be applied

Living Reviews in Solar Physics

http://www. livingreviews .org/lrsp-2011-6 
even for these impulsive flares. This further suggests that the plasmoid-induced-reconnection may play a key role in flares (see Section 4.1.6).

\subsection{Transient brightenings and X-ray jets}

Yohkoh/SXT has revealed that the corona is full of transient brightenings (Shimizu et al., 1992) and X-ray jets (Shibata et al., 1992a). Shimizu et al. (1994) investigates the structure of transient brightenings in active regions, and they found that these transient brightenings usually have single or multiple loop structure with the length of $0.5-4 \times 10^{4} \mathrm{~km}$. The total thermal energy estimated in a single transient brightening is $10^{25}-10^{29} \mathrm{erg}$ and the time scale is $1-10 \mathrm{~min}$. They further showed that the transient brightenings correlate with GOES C-class or sub-C class flares, suggesting that these brightenings are the spatially resolved soft X-ray counterpart of hard X-ray microflares (Lin et al., 1984). Later, Watanabe (1994) shows that the maximum temperature of sub-C class flares is the order of $10^{7} \mathrm{~K}$. The multiple loop structure of transient brightenings might be the evidence of magnetic reconnection via loop-loop interactions (Gold and Hoyle, 1960; Tajima et al., 1987; Sakai and Koide, 1992). Shimizu (1995) shows a simple scaling law between the number $N$ and total thermal energy $W$ of transient brightenings as follows:

$$
d N / d W \propto W^{-1.5 \sim-1.6},
$$

where $W$ ranges from $10^{27}$ to $10^{29} \mathrm{erg}$. Since this result is essentially the same as large flares and hard X-ray microflares (Hudson, 1991), it is likely that the same physical mechanism works for transient brightenings and flares.

X-ray jets are defined as soft X-ray brightenings with the shape of a collimated plasma outflow (Shibata et al., 1992a; see Figure 9). They are accompanied by small flares and X-ray bright points. The occurrence rate of X-ray jets is more than 20 per month between November 1991 and May 1992. Shimojo et al. (1996) shows that their average length and apparent velocity are $1.7 \times 10^{5} \mathrm{~km}$ and $200 \mathrm{~km} \mathrm{~s}^{-1}$, respectively. Shibata et al. (1992a) pointed out that an X-ray jet is often observed in an emerging flux region where emerging field might interact with preexisting field. Until now two types of X-ray jets have been identified (Figure 10):

(1) Two sided-loop jet: When emerging field interacts with the preexisting field that extends horizontally, jets are produced in the horizontal direction toward both sides of an emerging flux region.

(2) Anemone-type jet: When a newly emerging flux region appears in a unipolar region such as coronal holes, vertical jets are generated via the interaction of emerging field and the preexisting field that extends vertically. This forms an anemone-like loop structure in three-dimensional space (see the bottom panel of Figure 31). 


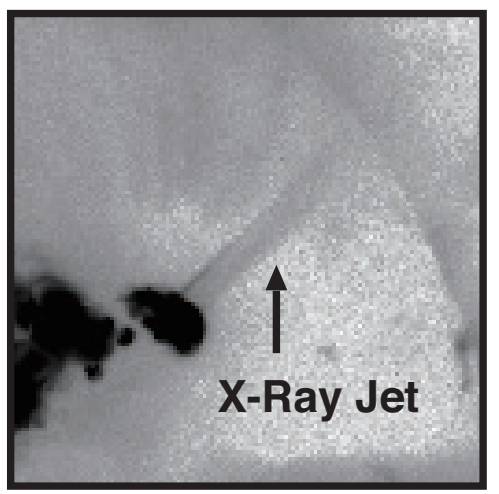

Yohkoh SXT Image 12-Nov-91 11:30UT

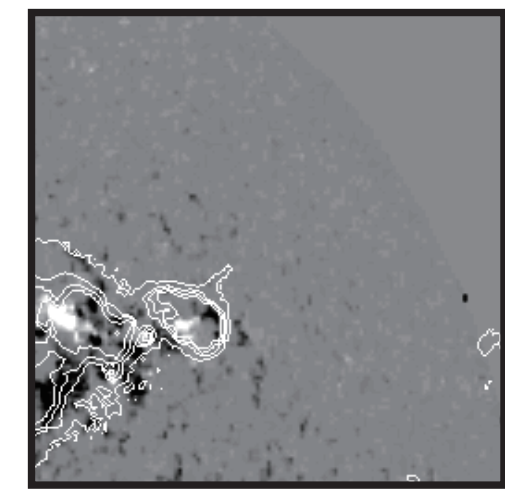

Kitt-Peak Magnetogram

12-Nov-91 16:07UT (contour Yohkoh SXT)

Figure 9: (Left) X-ray jet observed in soft X-rays (from Shibata et al., 1992a). Shown in reversed contrast. (Right) Longitudinal magnetic field distribution in the same field of view as in the left panel. The contour shows the soft X-ray intensity, revealing that the footpoint of the X-ray jet correspond to the mixed polarity region (from Shibata, 1999). 
(a)

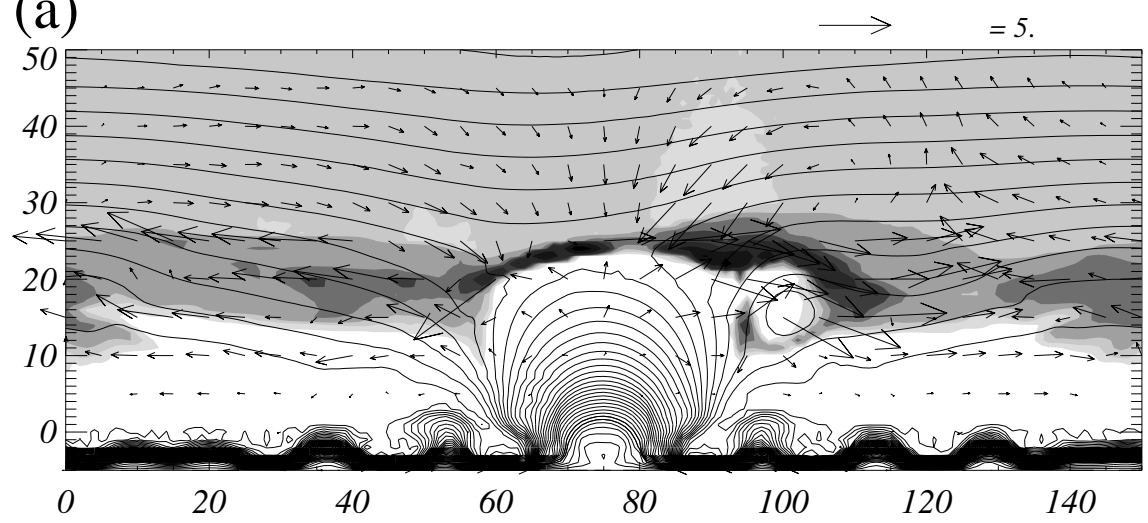

(b)

300
250
200
150
100
50
0

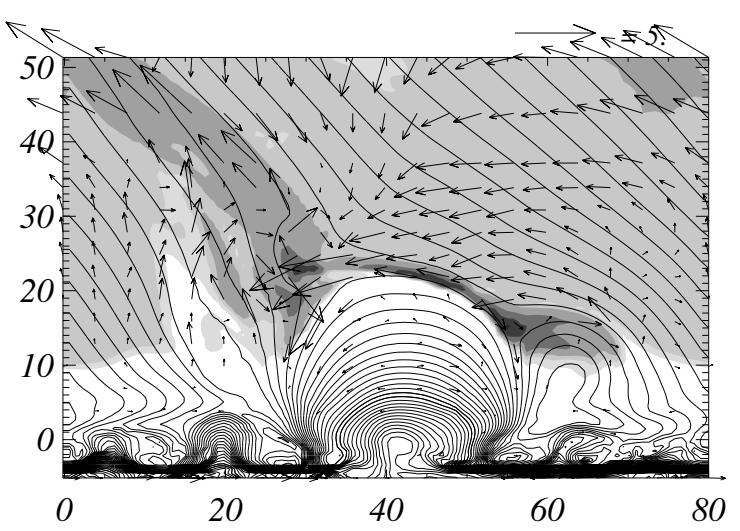

(c)
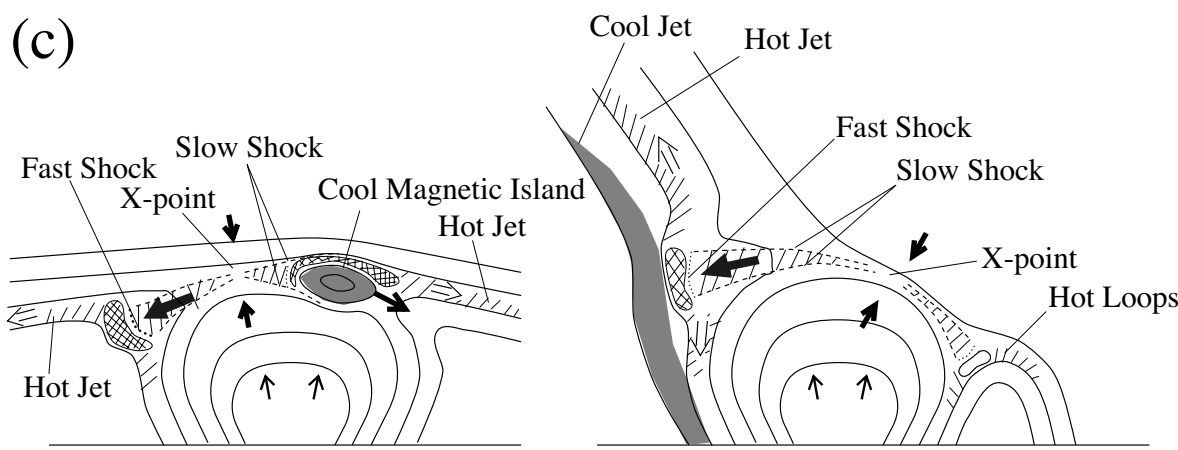

Figure 10: Two types of X-ray jets. (a) Two sided-loop jet. (b) Anemone-type jet. (c) Typical configuration for the two sided-loop jet at left panel and anemone-type jet at right panel (modified from Yokoyama and Shibata, 1996). 


\subsection{Unified model for flares, microflares, and jets}

Figure 11 illustrates a unified model that can explain large-scale flares, small-scale flares, flare-like phenomena accompanied by plasmoid ejection on the basis of magnetic reconnection (Shibata, 1999). In the case of large-scale flares, a plasmoid is located in a vertical current sheet formed inside a magnetic arcade, while in the case of small-scale flares, a plasmoid sits in a current sheet formed at the interface between emerging and preexisting fields. In both cases, the ejection of a plasmoid from a current sheet triggers fast magnetic reconnection, producing a flare and flare-like phenomenon (Section 4.1.6). Tables $1-4$ summarize the feature of flares and flare-like phenomena (Shimojo et al., 2007).
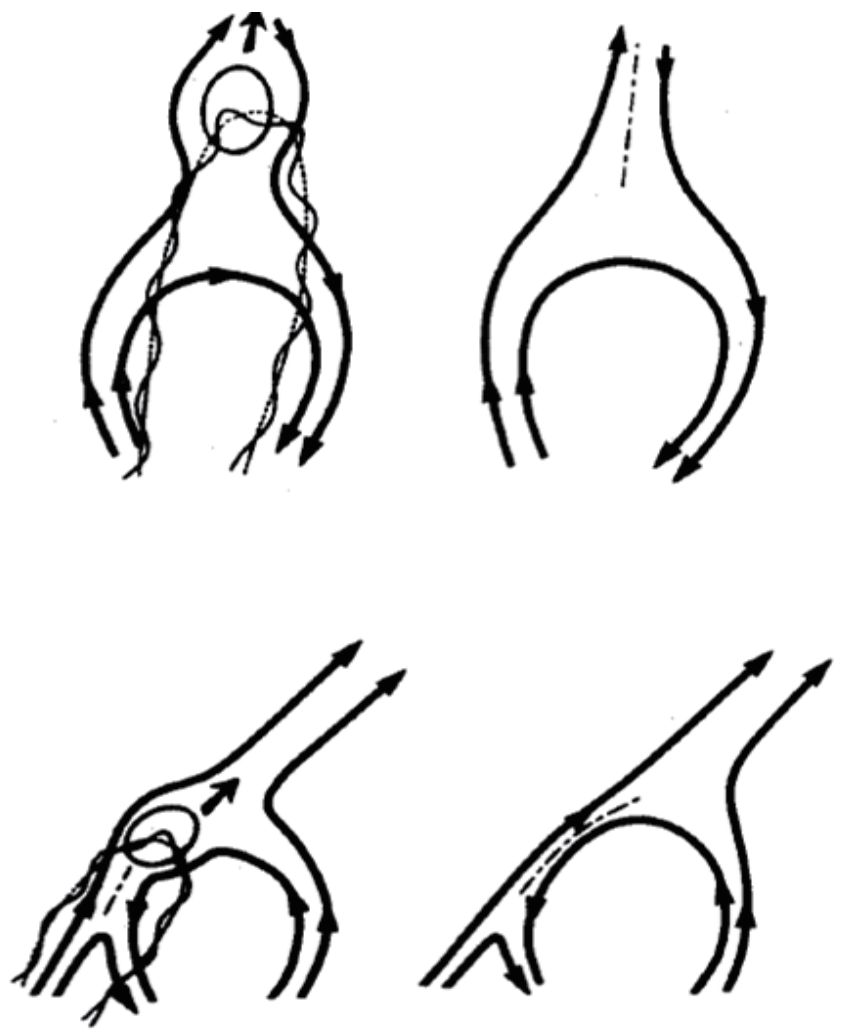

Figure 11: Unified model for solar eruptions (Shibata et al., 1995; Shibata, 1999). Top: Pre-eruption (left) and post-eruption (right) states of a large-scale eruption. Bottom: Pre-eruption (left) and post-eruption (right) states of a small-scale eruption.

\subsection{Avalanche model and non-reconnection models}

Observations revealed several interesting statistical properties of flares. First, the occurrence rate of flares decreases with the total energy from microflares to largest flares, following a power law (e.g., Lin et al., 1984; Dennis, 1985; Shimizu, 1995), as presented in Equation (4). The fact that the index of power-law is less than 2 suggests that microflares alone are insufficient to support the energy for coronal heating (Hudson, 1991), though the exact value of the power-law index is still controversial, especially for small events such as nanoflares (e.g., see Aschwanden, 2004). Even so, an universal power law seems to hold true for flares of various sizes suggests that there is a common physical mechanism operating in these different-scale flares.

Living Reviews in Solar Physics

http://www . livingreviews . org/lrsp-2011-6 
It is well known that the occurrence rate of earthquakes and the avalanches of a sandhill against their magnitude also show power law-like distributions, and these phenomena can now be understood in terms of self-organized criticality (Bak et al., 1987). Lu and Hamilton (1991) proposed that the coronal magnetic field is in a self-organized critical state, and solar flare represents the avalanche of many small reconnection events, which is analogous to the avalanche in the sandpile model. They successfully explained the observed power-law distribution of the occurrence rate of flares. Although there is a big gap between the avalanche model for a group of events and the magnetohydrodynamic model focused on an individual event, the avalanche model is still useful for understanding the process of energy release in the system of the solar atmosphere. If you want to know the recent development of the avalanche model, see the review by Charbonneau et al. (2001).

There have also been proposed several models for flares where magnetic reconnection is not assumed. One of them is found at the Alfvén's current disruption model (Alfvén and Carlqvist, 1967). The other models are proposed by Akasofu (1984), Uchida and Shibata (1988), Melrose (1997), and so on. Many of these models assume energy release inside a flaring loop, thus they are not consistent with those observations provided by Yohkoh, such as loop-top hard X-ray source and plasmoid ejection above a soft X-ray loop.

Table 1: Characteristics of flares and flare-like phenomena.

\begin{tabular}{lccc}
\hline & LDE flares & impulsive flares & microflares \\
\hline cusp configuration & yes $^{1}$ & no & $?$ \\
hot cusp loop & yes $^{1}$ & $?$ & $?$ \\
slow shock & ${\text { yes? })^{2}}^{2}$ & $?$ & $?$ \\
HXR loop-top source & no & yes $^{6}$ & $?$ \\
reconnection jet & no & no? & yes \\
plasmoid ejection & yes $^{3}$ & yes $^{8}$ & yes? \\
downflow & yes $^{4}$ & yes $^{9}$ & $?$ \\
inflow & yes $^{5}$ & $?$ & $?$ \\
\hline
\end{tabular}

1 Tsuneta et al. (1992a), Tsuneta (1996), Forbes and Acton (1996).

2 Tsuneta (1996)

3 Hudson (1994), Yokoyama et al. (2001), Kim et al. (2005).

${ }^{4}$ McKenzie and Hudson (1999), McKenzie (2000), Innes et al. (2003), Sui and Holman (2003).

${ }^{5}$ Lin et al. (2005), Hara et al. (2006), Narukage and Shibata (2006).

6 Masuda (1994).

7 Wang et al. (2007).

8 Shibata et al. (1995), Tsuneta (1997), Ohyama and Shibata (1997, 1998), Kim et al. (2005).

${ }^{9}$ Asai et al. (2004), Linton and Longcope (2006), TanDokoro and Fujimoto (2005). 
Table 2: Comparison of scales and associated mass ejection.

\begin{tabular}{|c|c|c|c|c|}
\hline & $\begin{array}{l}\text { size }(L) \\
\left(10^{4} \mathrm{~km}\right)\end{array}$ & $\begin{array}{l}\text { time scale }(t) \\
(\mathrm{s})\end{array}$ & $\begin{array}{l}\text { energy } \\
\text { (erg) }\end{array}$ & associated ejection \\
\hline $\begin{array}{l}\text { microflares } \\
\text { (ARTBs) }\end{array}$ & $0.5-4$ & $60-600$ & $10^{26}-10^{29}$ & jet/surge \\
\hline impulsive flares & $1-10$ & $60-3 \times 10^{3}$ & $10^{29}-10^{32}$ & $\begin{array}{l}\text { X-ray } / \mathrm{H} \alpha \\
\text { filament eruption }\end{array}$ \\
\hline LDE flares & $10-40$ & $3 \times 10^{3}-10^{5}$ & $10^{30}-10^{32}$ & $\begin{array}{l}\mathrm{X} \text {-ray } / \mathrm{H} \alpha \\
\text { filament eruption }\end{array}$ \\
\hline $\begin{array}{l}\text { large scale } \\
\text { arcade formation }\end{array}$ & $30-100$ & $10^{4}-2 \times 10^{5}$ & $10^{29}-10^{32}$ & $\begin{array}{l}\text { X-ray } / \mathrm{H} \alpha \\
\text { filament eruption }\end{array}$ \\
\hline
\end{tabular}

Table 3: Comparison of physical quantities.

\begin{tabular}{llllll}
\hline & $\begin{array}{l}B \\
(\mathrm{G})\end{array}$ & $\begin{array}{l}n_{e} \\
\left(\mathrm{~cm}^{-3}\right)\end{array}$ & $\begin{array}{l}V_{A} \\
\left(\mathrm{~km} \mathrm{~s}^{-1}\right)\end{array}$ & $\begin{array}{l}t_{A}=L / V_{A} \\
(\mathrm{~s})\end{array}$ & $t / t_{A}$ \\
\hline microflares & 100 & $10^{10}$ & 3000 & 5 & $12-120$ \\
impulsive flares & 100 & $10^{10}$ & 3000 & 10 & $6-300$ \\
LDE flares & 30 & $2 \times 10^{9}$ & 2000 & 90 & $30-10^{3}$ \\
large scale & 10 & $3 \times 10^{8}$ & 1500 & 400 & $25-500$ \\
arcade formation & & & & & \\
\hline
\end{tabular}

Table 4: Unified view of flares and flare-like phenomena.

\begin{tabular}{lll}
\hline & $\begin{array}{l}\text { mass ejections } \\
(\text { cool })\end{array}$ & $\begin{array}{l}\text { mass ejections } \\
\text { (hot) }\end{array}$ \\
\hline giant arcades & $\begin{array}{l}\text { H } \alpha \text { filament } \\
\text { eruptions }\end{array}$ & CMEs \\
LDE flares & $\begin{array}{l}\text { H } \alpha \text { filament } \\
\text { eruptions }\end{array}$ & $\begin{array}{l}\text { X-ray plasmoid } \\
\text { ejections/CMEs }\end{array}$ \\
& H $\alpha$ sprays & $\begin{array}{l}\text { X-ray plasmoid } \\
\text { ejections }\end{array}$ \\
impulsive flares & H $\alpha$ surges & X-ray jets \\
transient brightenings (microflares) & surges/spicules & EUV jets \\
$\begin{array}{l}\text { EUV microflares } \\
\text { facular points } \\
\text { (nanoflares?) }\end{array}$ & spicules & (Alfvén waves) \\
\hline
\end{tabular}




\section{$3 \quad$ Energy Build-up}

The evolution of a flare basically starts with the emergence of magnetic field into the surface (flux emergence), which carries magnetic energy from the interior to the atmosphere. Part of this magnetic energy is immediately released when emerging magnetic field expands to form a magnetic structure on the Sun. The electric current crossing the magnetic field (cross-field current) generate the Lorentz force, so it drives expansion. The electric current flowing along magnetic field (fieldaligned current), on the other hand, does not generate the Lorentz force, so the field-aligned current is not used during an expanding process. In addition to that, the field-aligned current is not easy to dissipate in a highly conductive medium such as the solar corona, so it is stored there as free energy that becomes the energy source of flares and flare-associated phenomena.

\subsection{Emergence of magnetic field (flux emergence)}

In this section we start with the morphology of flux emergence, then explain the dynamic nature of flux emergence and the characteristics of magnetic structures formed via flux emergence.

\subsubsection{Morphology}

Morphologically speaking, the field-aligned current introduces distortion to a magnetic structure where magnetic field lines tend to be aligned with the so-called polarity inversion line defined as the boundary between positive and negative polarity regions on the surface. When there is no field-aligned current, and when the inversion line is nearly straight, field lines overlie the inversion line transversely, forming a potential field without any free energy. The configuration of magnetic field therefore indicates whether field-aligned current (or free energy) exist or not in a magnetic structure (it is not generally true that field lines overlie the inversion line transversely in a potential field; for example, if the inversion line is bent, the angle between field lines and the inversion line deviates significantly from $90^{\circ}$, while there are cases with field lines locally almost parallel to the inversion line in quadrupolar configurations).

The morphology of flux emergence has been studied by observing emerging flux regions (EFRs) on the Sun (Kurokawa, 1987; Tanaka, 1991; Leka et al., 1996; Strous et al., 1996; Ishii et al., 1998; Otsuji et al., 2007). Observations show that an arch filament system (AFS) appears in the early phase of flux emergence (Figure 12a). The top of an AFS rises at about $10 \mathrm{~km} \mathrm{~s}^{-1}$ in a chromospheric level (Bruzek, 1969; Chou and Zirin, 1988), while slower rising motions (about 0.1$1 \mathrm{~km} \mathrm{~s}^{-1}$ ) have been observed in the photosphere (Tarbell et al., 1988). In the late phase of flux emergence, a dark filament is sometimes observed above the polarity inversion line, suggesting that a sheared magnetic structure is formed (see Section 3.2.1). Figure 12 shows the evolution of an active region observed in white light and $\mathrm{H} \alpha$. An important result from those observations is that the emerging magnetic field is less sheared in the early phase of flux emergence, while a sheared structure develops in the late phase.

The evolution of EFRs observed on the Sun provides the key information on the subsurface structure of emerging magnetic field. As we mentioned in the introduction, it has been suggested that the magnetic field is confined to form thin flux tubes in the convection zone. The swirling motions of convective plasma in helical turbulence might add some twist to these flux tubes (Longcope et al., 1998), and the twisted field lines naturally generate the field-aligned current. Also flux tubes might be twisted enough to keep their coherence when they rise through the convection zone (Emonet and Moreno-Insertis, 1998; Cheung et al., 2006; Fan, 2008). An idealized model of such a twisted flux tube is the so-called Gold-Hoyle flux tube (Gold and Hoyle, 1960), in which field lines are uniformly twisted while the current density takes the highest value at the axis of the flux tube and decreases toward the boundary of the flux tube. Assuming that such a twisted flux tube emerges into the surface, part of the flux tube with less field-aligned current first appears and 
DEVELOPMENT OF MAGNETIC SHEAR

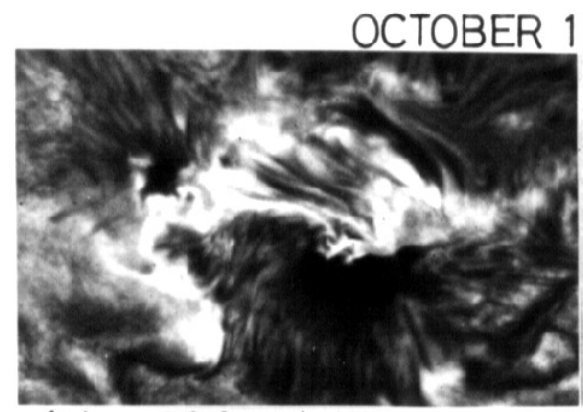

$11-13,1981$
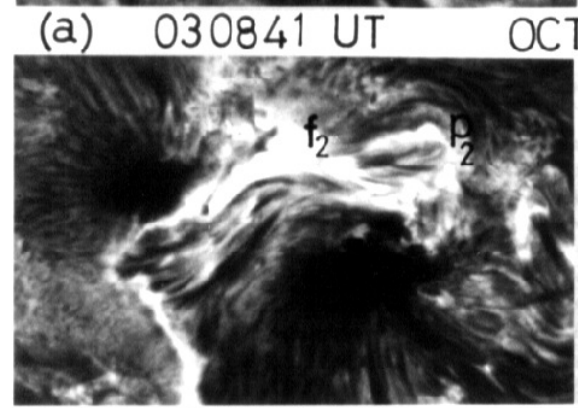

OCT. 11 (b) 011000 UT
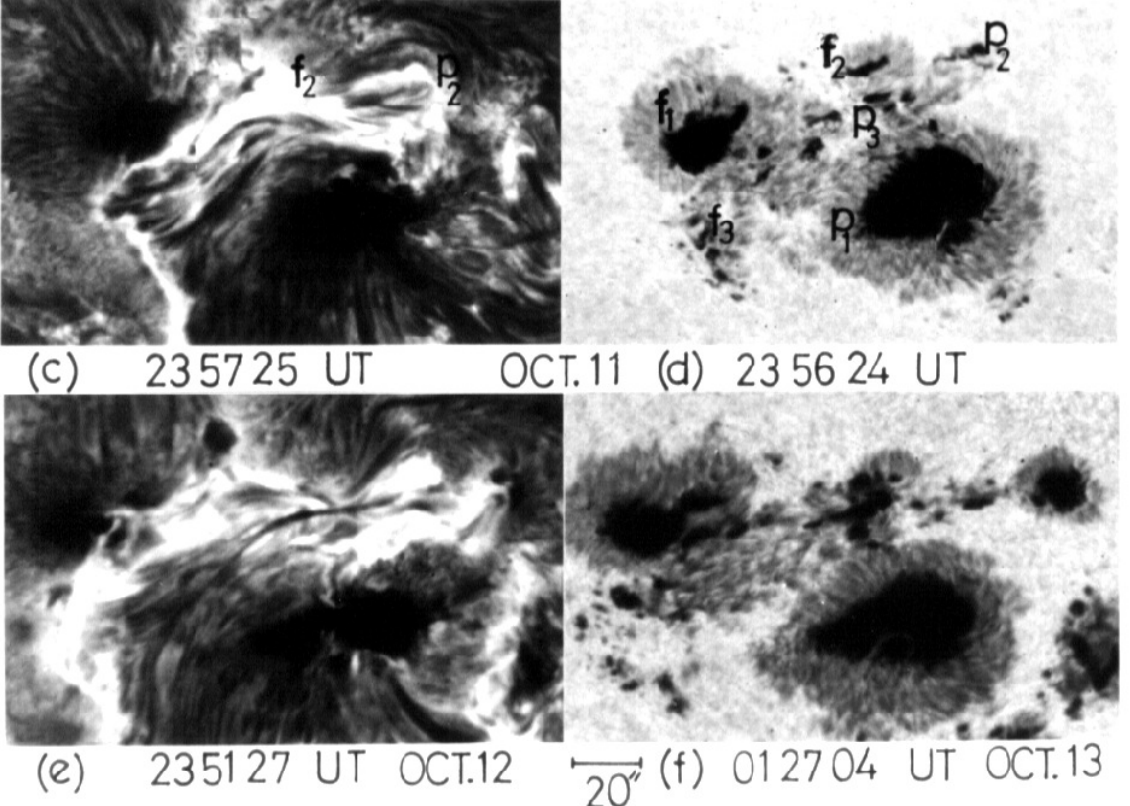

Figure 12: Evolution of an active region, showing the development of magnetic shear. The left panels are $\mathrm{H} \alpha$ images and the right panels are white-light images (from Kurokawa, 1989). 
forms a potential field-like structure, which is reminiscent of an AFS observed on the surface. As emergence proceeds, inner central part of the flux tube that contains strong field-aligned current appears, forming a sheared arcade. This thought experiment presents a scenario of forming a sheared magnetic structure with free energy in the corona. The dynamic process suggested by this scenario will be discussed in the succeeding sections.

\subsubsection{Dynamics}

Recently, much efforts have been made to clarify the dynamic nature of flux emergence using numerical simulations. These simulations had first been performed in two dimension (see Figure 13). Shibata et al. (1989a) performed simulations in a flux-sheet configuration to reproduce several key features of emerging magnetic field and associated flow. They derived a self-similar solution expressing the expansion of emerging magnetic field (Shibata et al., 1989b, 1990b; Tajima and Shibata, 1997), which is driven by the Parker (i.e., buoyancy) instability (Parker, 1955). The solution shows how the rise velocity $\left(v_{z}\right)$ and gas density $(\rho)$ of plasma, and the strength of horizontal magnetic field $\left(B_{h}\right)$ depend on height, given by

$$
v_{z} \sim \omega_{n} z, \rho \propto z^{-4}, B_{h} \propto z^{-1} .
$$

Here, $z$ is the height and $\omega_{n}$ represents the growth rate in the nonlinear phase of the Parker instability, given by

$$
\omega_{n} \sim \frac{1}{2} \omega_{l} \simeq 0.1\left(1+2 \beta_{*}\right)^{-1 / 2} \frac{c_{s}}{\Lambda},
$$

where $\omega_{l}, \beta_{*}, c_{s}$, and $\Lambda$ are the linear growth rate of the instability, plasma beta, adiabatic sound velocity, and pressure scale height of the flux sheet initially assumed. The solution explains that the drain of mass along magnetic field evacuates magnetic loops where the gas density decreases with height while both the rise velocity and Alfvén velocity increases with height. A comparison of this analytic result to an MHD simulation is given in Figure 13.

Similar two-dimensional simulations have been performed by Nozawa et al. (1992) to study the effect of convection on flux emergence. Shibata et al. (1990a) studied the convective collapse (Parker, 1978; Spruit and Zweibel, 1979) that occurs at photospheric footpoints of emerged loops, showing that the field strength becomes a kilo Gauss at the footpoints. The interaction of emerging and preexisting coronal fields has also been investigated by Yokoyama and Shibata (1996), which is further developed by Miyagoshi and Yokoyama (2004) where thermal conduction is taken into account.

Another type of two-dimensional simulations of flux emergence has also been done in a fluxtube configuration (Krall et al., 1998; Magara, 2001). Magara (2001) demonstrates that a flux tube rising through the convection zone becomes flattened when it approaches the surface where the nature of the background gas layer changes from a convectively unstable state to a stable one. This is because when the top part of a flux tube comes close to the surface, it stops rising while the bottom part still continues to rise, making the flux tube extend horizontally to form a flux sheet-like structure just below the surface (see the middle panel of Figure 14a). At the same time, the mass contained in the flux sheet-like structure is squeezed out, locally reducing the density below the surface. By applying the concept of the Rayleigh-Taylor instability to this region (high density region lies on a flux sheet with low gas density), we show that the flux sheet can emerge when its horizontal extent becomes greater than the critical wavelength (see Figure 14b), which is given by

$$
\lambda=4 \pi \frac{c_{s, i}^{2}}{g},
$$

where $c_{s, i}$ and $g$ are the photospheric isothermal sound velocity and gravitational acceleration. This length is about $2 \mathrm{Mm}$ in the photosphere, and observations also suggested that there is a 


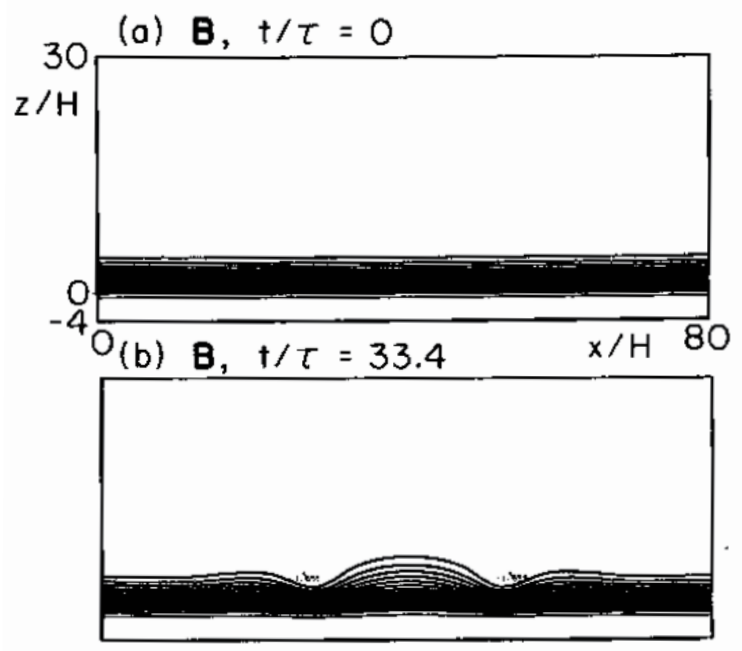

(c) $B, t / \tau=49.7$
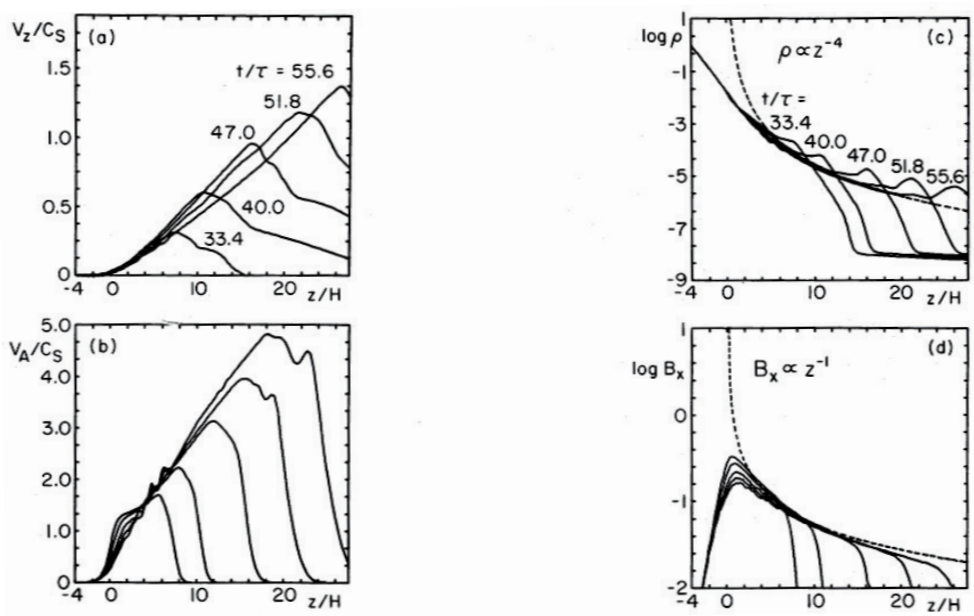

Figure 13: Top: Two-dimensional simulations of flux emergence in a flux-sheet configuration. Contours represent magnetic field lines. Bottom: Distributions of rising velocity $\left(v_{z}\right)$, Alfvén velocity $\left(v_{A}\right)$, density $(\rho)$, and horizontal magnetic field $\left(B_{x}\right)$ with height during the expansion of emerging magnetic field. The dashed curves in the graphs (c) and (d) at the bottom panel represent the analytic curves of the self-similar solution (from Shibata et al., 1989b). 
a)
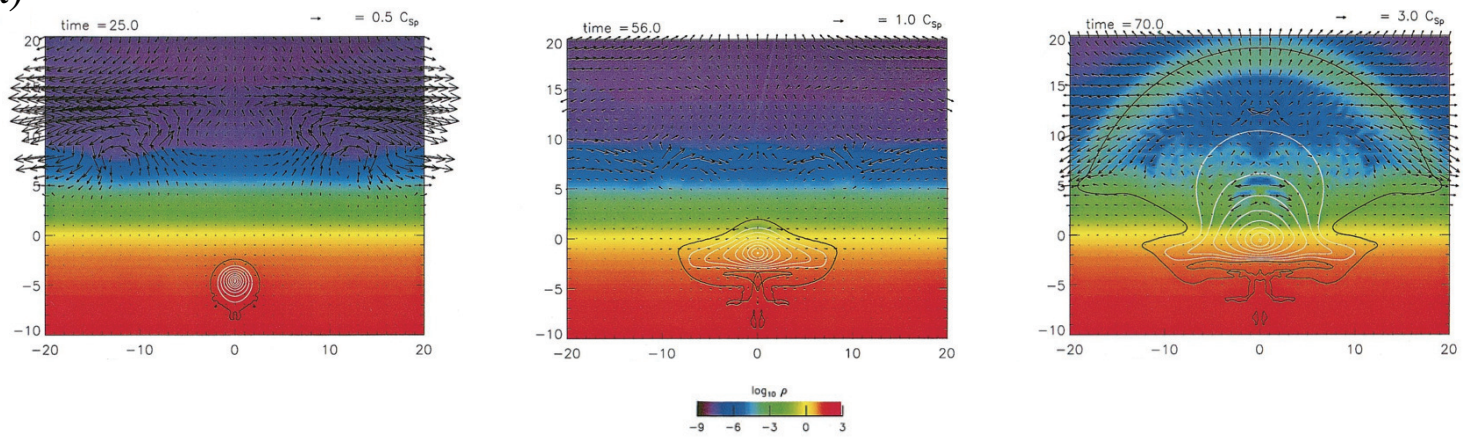

b)

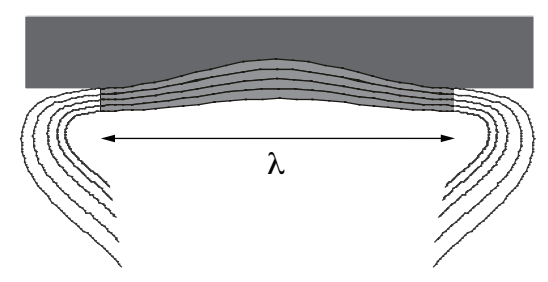

c)

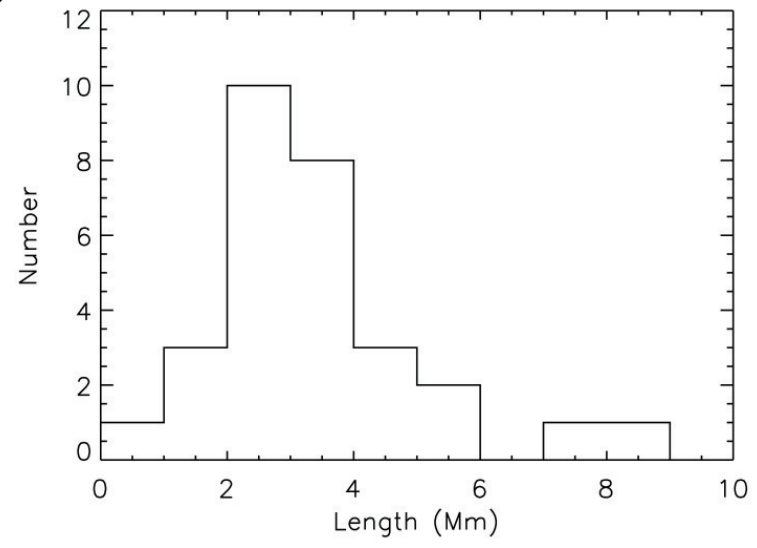

Figure 14: (a) A rising flux tube becomes flattened when it approaches the surface. (b) A developed magnetic sheet and its horizontal extent $(\lambda)$ (from Magara, 2001). (c) Histogram of the observed horizontal extent of emerging magnetic field (from Pariat et al., 2004). 
threshold of the horizontal extent of emerging magnetic field which is about $2 \mathrm{Mm}$ (Pariat et al., 2004, see Figure 14c). A more precise analysis of successful emergence has been obtained by taking the stratification of magnetic field into account, that is,

$$
-\Lambda \frac{\partial}{\partial z} \ln B>-\frac{\gamma}{2} \beta \delta+k_{/ /}^{2}\left(1+\frac{k_{\perp}^{2}}{k_{z}^{2}}\right),
$$

where $\Lambda, \gamma, \beta$, and $\delta=\frac{\partial \ln T}{\partial \ln P}-\left(\frac{\partial \ln T}{\partial \ln P}\right)_{a d}$ are the photospheric pressure scale height, ratio of specific heat, plasma beta, and excess of superadiabaticity, while $k_{/ /}$and $k_{\perp}$ are the wavenumbers in two horizontal directions in unit of local scale height (the former is the field-aligned component and the latter the cross-field component) and $k_{z}$ is the wavenumber in the vertical direction (Newcomb, 1961; Acheson, 1979). Using this formula, Archontis et al. (2004) and Murray and Hood (2008) have made an extended survey of successful emergence.
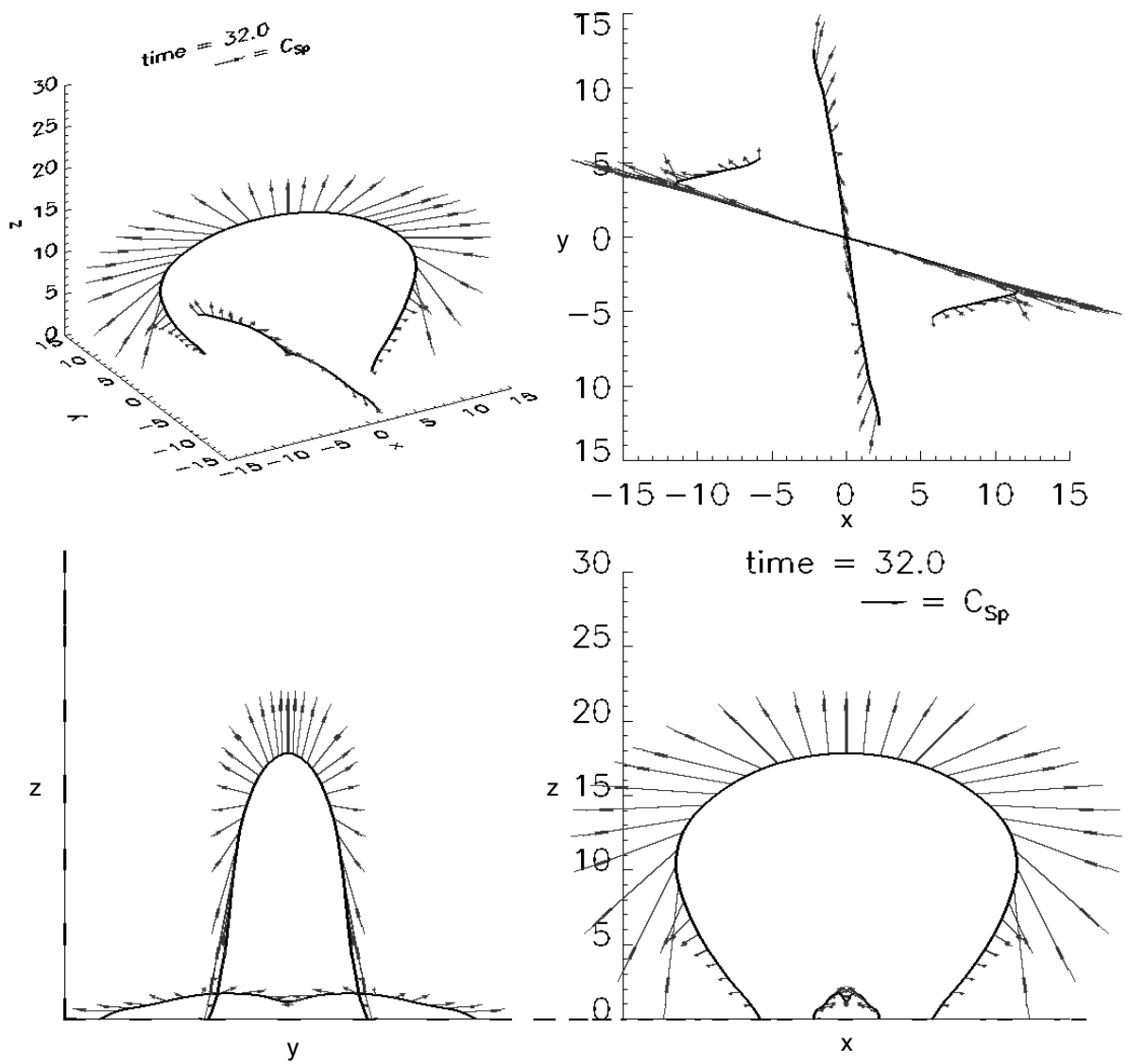

Figure 15: A magnetic structure formed by the emergence of a twisted flux tube. Two field lines (outer and inner) composing the flux tube are presented. The arrows on these field lines represent flow velocity. The outer field line forms an expanding loop, below which the inner field line forms a sheared loop which is less dynamic (from Magara and Longcope, 2003).

The emergence in a flux-tube configuration is significantly different from the emergence in a flux-sheet configuration. In a flux-tube configuration field lines have different geometric shapes depending on their locations inside the flux tube, and this causes the difference in evolution among 
these field lines. To see how different it is, we should know the relation between the dynamic nature and geometrical shape of emerging field lines, which is demonstrated below.

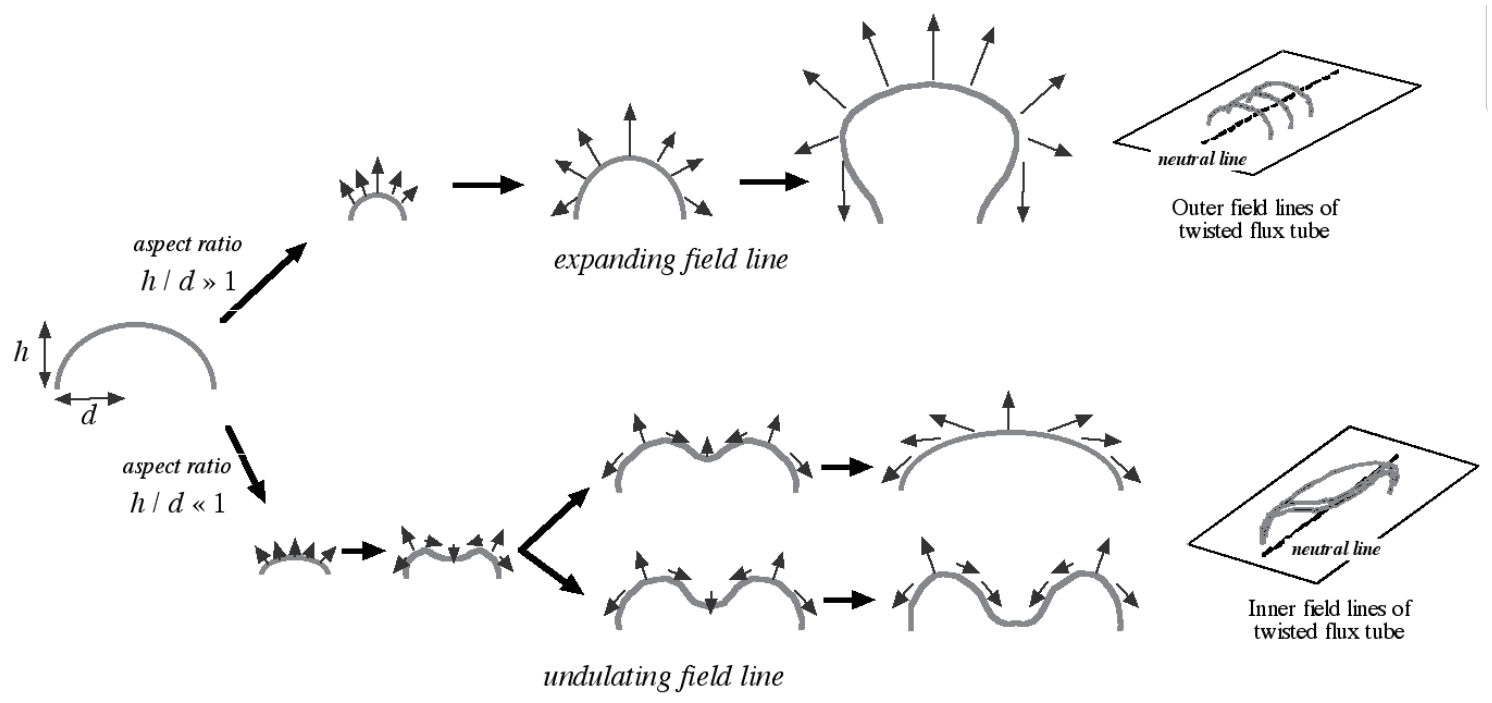

Figure 16: Difference in evolution between the outer and inner field lines composing a twisted flux tube (from Magara and Longcope, 2003).

The outer field lines composing a twisted flux tube, which are located near the boundary of the flux tube, have helical structure, while the inner field lines close to tube axis have a strong axial component. This geometrical difference between the outer and inner field lines causes different dynamic behavior of these field lines after they emerge (Magara and Longcope, 2003, see Figure 15). Figure 16 schematically explains this. The outer field lines form $\Omega$-loops with a large aspect ratio of height to footpoint separation on the surface, while the inner field lines form relatively flat $\Omega$-loops with a smaller aspect ratio than the outer field lines. In the outer field lines, a diverging downflow is strong because they have large curvature at the top so that the gravity works effectively, enhancing magnetic buoyancy and making these field lines continuously expand. On the other hand, a diverging flow is weak along the inner field lines in a flatter shape, where sometimes the mass even accumulates somewhere at the field line, forming dipped structure. Accordingly, while the outer field lines form a continuously expanding arcade, the inner field lines form a quasi-static structure below the overlying arcade.

A quantitative analysis on the dynamic behavior of emerging field lines in a flux-tube configuration has been made by Magara (2004). The rise velocity of a field line with the curvature $\kappa$ at the top is given by

$$
\left\langle v_{\text {rise }}\right\rangle=\frac{\Lambda_{m}}{2} \sqrt{g \kappa},
$$

where $\left\langle v_{\text {rise }}\right\rangle$ means an average in time, $\Lambda_{m}$ is the scale height of magnetic-field strength and $g$ is the gravitational acceleration. This indicates that the rise velocity of an emerging field line depends on a geometrical property (curvature) as well as stratification of magnetic field.

\subsubsection{Latest progress}

Continuously increasing computational power enables to investigate flux emergence in the three dimension. Fan (2001) simulated the pattern of surface flows driven by the emergence of a twisted flux tube and compared it with observations. Abbett and Fisher (2003) present an integrated 
simulation where a subsurface convection model and a coronal model are combined. They have confirmed that emerging magnetic field tends to be relaxed to a force-free field state in the chromosphere and corona. Nozawa (2005), Murray et al. (2006) and Murray and Hood (2007, 2008) have done an extended survey of flux emergence by changing the subsurface configuration of magnetic field.

One of the issues related to flux emergence is the behavior of the axis of an emerging flux tube. It can be expected that the emergence of the axis becomes easy when the axis is strongly bent and has an $\Omega$ shape because the mass drains efficiently along the axis, thereby enhancing buoyancy. This conjecture has been confirmed by a series of works: in Magara (2001) which keeps a straight axis in the horizontal direction (2.5-dimensional simulation), the axis does not emerge (see Figure 14a), while when a flux tube is assumed to have a curved axis, the axis emerges. In fact, the emergence of the axis proceeds more efficiently when a curved (convex-up) flux tube is initially assumed (Hood et al., 2009; MacTaggart and Hood, 2009). Archontis et al. (2004, 2005, 2007), Isobe et al. (2005, 2006), and Galsgaard et al. (2005, 2007) have studied the interaction of emerging and preexisting fields in various three-dimensional configurations (see Section 4.3). A series of works done by Manchester (Manchester IV, 2001; Manchester IV et al., 2004; Manchester IV, 2007) have shown the origin of shear flows observed on the surface (see Section 4.3). Recently, studies taking realistic factors into account such as radiation, thermal conduction, viscosity and partial ionization, have enabled to make a detailed comparison between simulations and observations (Leake and Arber, 2006; Cheung et al., 2007, 2008; Abbett, 2007; Hansteen et al., 2007).

\subsection{Magnetic structure}

Flux emergence is an essential process by which a magnetic structure containing free energy is formed on the Sun. It is still difficult to grasp the whole magnetic structure formed on the Sun observationally, while parts of the structure can be deduced from observed objects such as filament (or equivalently prominence which is observed on the limb of the Sun) and sigmoid that shows 'S' or 'inverse-S' shape in soft X-rays (Pevtsov et al., 1995; Rust and Kumar, 1996; Canfield et al., 1999). Modeling of these objects is therefore a key to the understanding of the magnetic structure related to flares.

We here try to understand the nature of such magnetic structure by referring to filament/prominence and sigmoid, both of which are observed before the onset of a flare (precursor), where an emphasis is put on their formation processes. We also explain how to reconstruct invisible magnetic structure using surface magnetic field which is observed. Force-free-field modeling is one of the possible methods of reconstruction. We also explain a famous conjecture on the energy state of force-free field, which is known as Aly-Sturrock conjecture.

\subsubsection{Filament (Prominence)}

Observations have revealed various structural features of filament (Martin, 1990, 1998; Schmieder et al., 2006; Rust and Kumar, 1994). A filament tends to form around the polarity inversion line separating opposite polarity regions (main polarity regions), forming a filament channel. Along the inversion line is observed the main body of a filament, which is called 'spine'. There are also small weak-flux regions distributed in a filament channel (Martin, 1998; Chae et al., 2001), and these regions, which are called parasitic or satellite polarity regions compared to the main polarity regions, contribute to forming secondary structure such as filament feet called 'barbs'. An important result about parasitic polarity regions is that these regions have the opposite polarity to the nearby main polarity region (Martin et al., 1994), and field lines connecting to parasitic polarity regions are suggested to have dipped structure (Aulanier and Démoulin, 1998; Aulanier et al., 1998; López Ariste et al., 2006).

Living Reviews in Solar Physics

http://www. livingreviews.org/lrsp-2011-6 

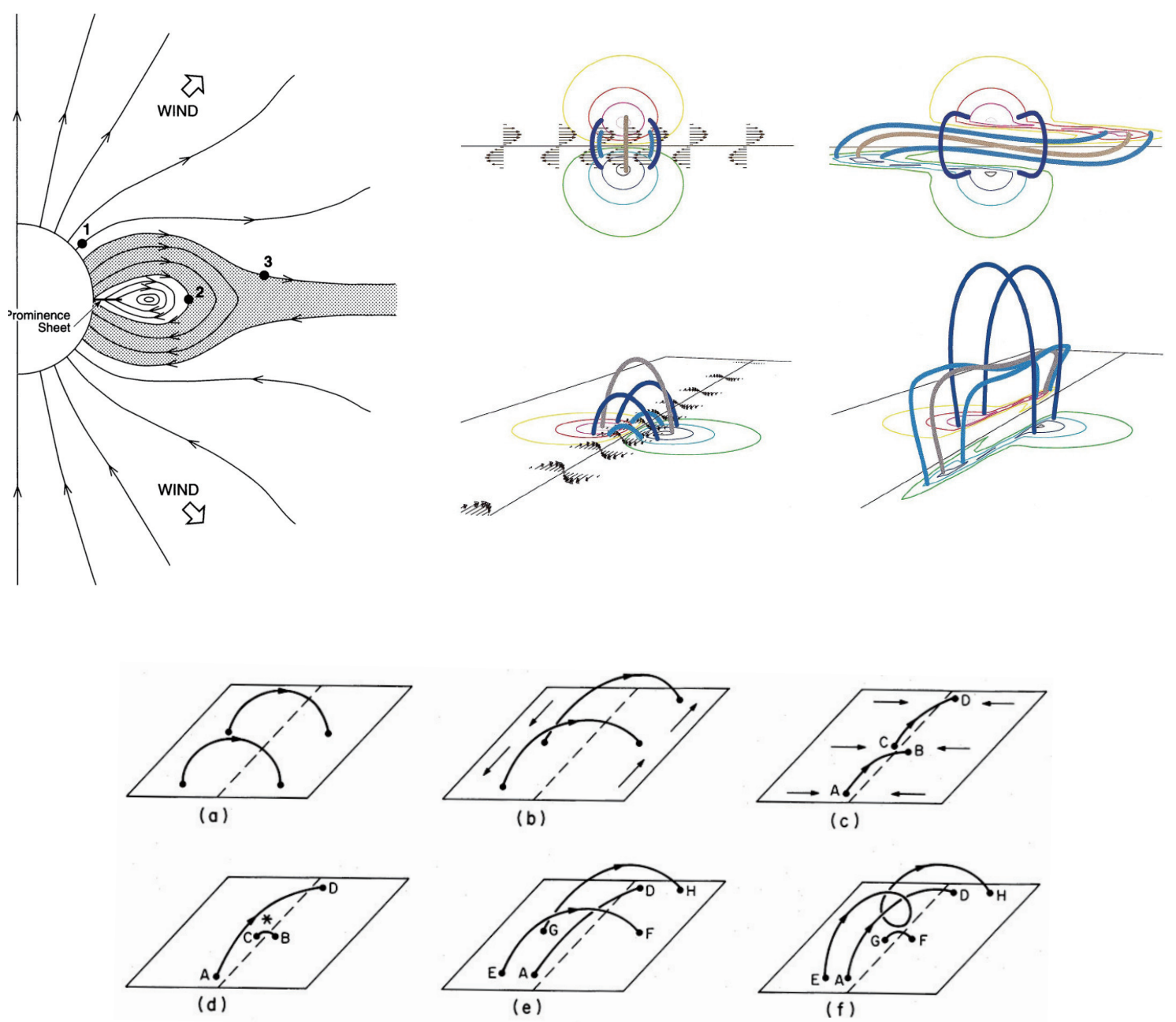

Figure 17: Magnetic structure of a filament (from Low and Hundhausen, 1995) (top-left panel), DeVore and Antiochos (2000) (top-right panel) and van Ballegooijen and Martens (1989) (bottom panel). 
There is also known a hemispheric chirality rule of filaments: 'dextral' filaments tend to appear in the northern hemisphere where the magnetic field with left-handed twist is preferentially observed, while 'sinistral' filaments are frequently observed in the southern hemisphere where the right-handed twist is dominant (Rust and Kumar, 1994; Martin, 1998; Pevtsov et al., 2003).

Theoretical studies of the magnetic structure containing a filament has been done extensively. Low and Hundhausen (1995) and Low (1996) suggest that a twisted flux tube emerges via magnetic buoyancy to form a flux rope above the surface, inside which a filament is formed (Figure 17, topleft panel). Antiochos et al. (1994) and DeVore and Antiochos (2000) demonstrate that a sheared arcade contains a filament, which is formed by shear flows around polarity inversion line (Figure 17 top-right panel). van Ballegooijen and Martens (1989) presents a result showing that shear flows followed by converging flows toward polarity inversion line causes reconnection at the surface (called 'flux cancellation'), which creates a twisted flux rope inside which a filament is formed (Figure 17 bottom panel). The last one has been developed later to explain the global hemispheric pattern of filaments (van Ballegooijen et al., 2000; Mackay and van Ballegooijen, 2005). The origins of these surface motions and possible relation between twist and chirality mentioned above have been studied from the viewpoint of flux emergence. Recently, Magara (2007) shows that the emergence of a twisted flux tube undulating along tube axis naturally reproduces those observed structural features (Figure 18). Field lines close to tube axis (inner field lines) form the main body of a filament. On the other hand, field lines composing the twisted flux tube which are located away from tube axis (outer field lines) form a coronal arcade that overlies the main body of a filament, while they underlie the main body by forming barbs. Regarding the chirality of filaments, this work suggests that a flux tube of left-handed (right-handed) twist tends to form a dextral (sinistral) filament (see the middle panels in Figure 18). Also, it was shown that the emergence of U-loops distributed below the axis causes apparent flux cancellation on the surface (Magara, 2011). In this respect, the evolution of U-loops has been observed and analyzed by van Driel-Gesztelyi et al. (2000). They show the peculiar motions of magnetic polarities on the surface and suggest that flux cancelation proceeds without significant energy release.

Although there are some observations suggesting that a filament is formed via the emergence of a twisted flux tube (Lites, 2005; Okamoto et al., 2008), it should be mentioned that a number of filaments are formed away from emerging active regions. These filaments form along the polarity inversion line of decaying active regions, in between active regions, and even in the polar regions (polar crown filaments).

Recently, the dynamic nature of filament/prominence has well been captured with advanced observing tools, which provides the detailed information on plasma motions in a filament/prominence (Berger et al., 2008; Okamoto et al., 2007). The modeling focused on the dynamic nature of filament/prominence has also been reported (Antiochos et al., 1999b; Karpen and Antiochos, 2008).

\subsubsection{Sigmoid}

Sigmoid is observed as either S or inverse S-shaped structure with soft X-ray enhancement in the corona, and it has been known as the precursor of a big cusp-shaped flare (Tsuneta et al., 1992a) or coronal mass ejection (CME) (Canfield et al., 1999; Sterling and Hudson, 1997). Recently, using the soft X-ray observations by Hinode, McKenzie and Canfield (2008) found that sigmoid is not a single loop but consists of many loops.

Gibson et al. (2002) use linear force-free field modeling to analyze an observed sigmoid. Pevtsov (2002) shows an interesting result on the spatial relationship between a filament and a sigmoid. Régnier and Amari (2004) use nonlinear force-free field modeling to study a global magnetic structure containing a filament, sigmoid, and a large $\Omega$-loop overlying the filament and sigmoid. They explain that the filament and sigmoid are composed of the loops that have a smaller aspect ratio of height to footpoint separation than the overlying $\Omega$-loop. This result suggests that a filament and

Living Reviews in Solar Physics

http://www . livingreviews . org/lrsp-2011-6 

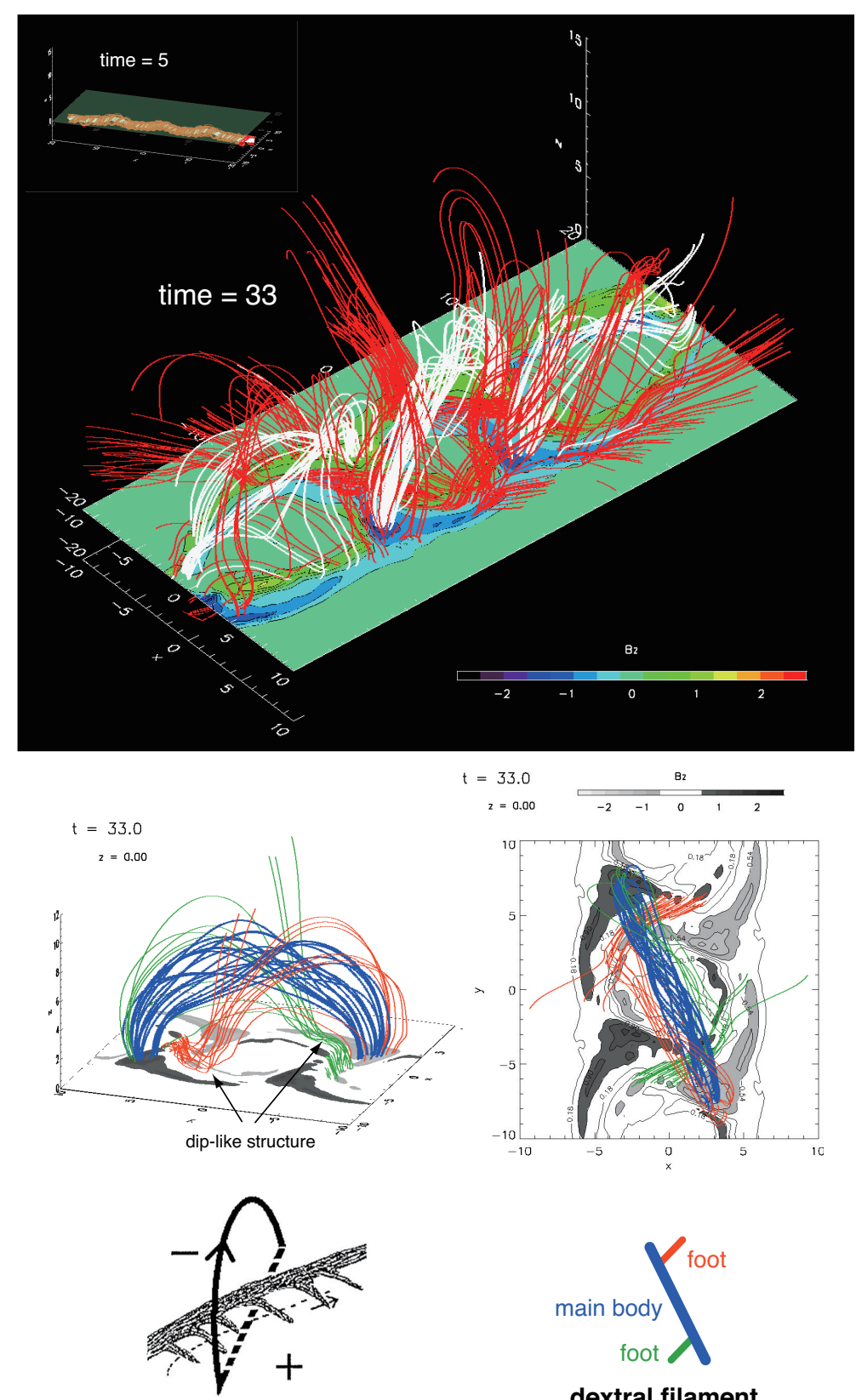

Left-Skewed

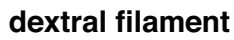

Figure 18: Possible magnetic structure of a filament formed via the emergence of a twisted flux tube of left handedness. The top panel shows pre- and post-emergence states. The flux tube undulates along the axis when it emerges. Field lines composing the twisted flux tube which are located away from tube axis (outer field lines) are displayed in red, while inner field lines close to tube axis are in white. Note that the inner field lines form the main body of a filament while the outer field lines overlie the main body by forming a coronal arcade and underlie the main body by forming barbs. The middle panels show top and side views of the main body formed by the inner field lines (blue) and barbs formed by the outer field lines (green and orange). The bottom panels schematically show the spatial relationship among the coronal arcade, main body and barbs of a dextral filament. The sinistral case is given by the mirror symmetry of the dextral case (flux tube has right handedness). From Magara (2007), except for the top panel, which is now given from a different viewing angle (same 3D plot), and the bottom-left panel, which is adapted from Martin (1998). 


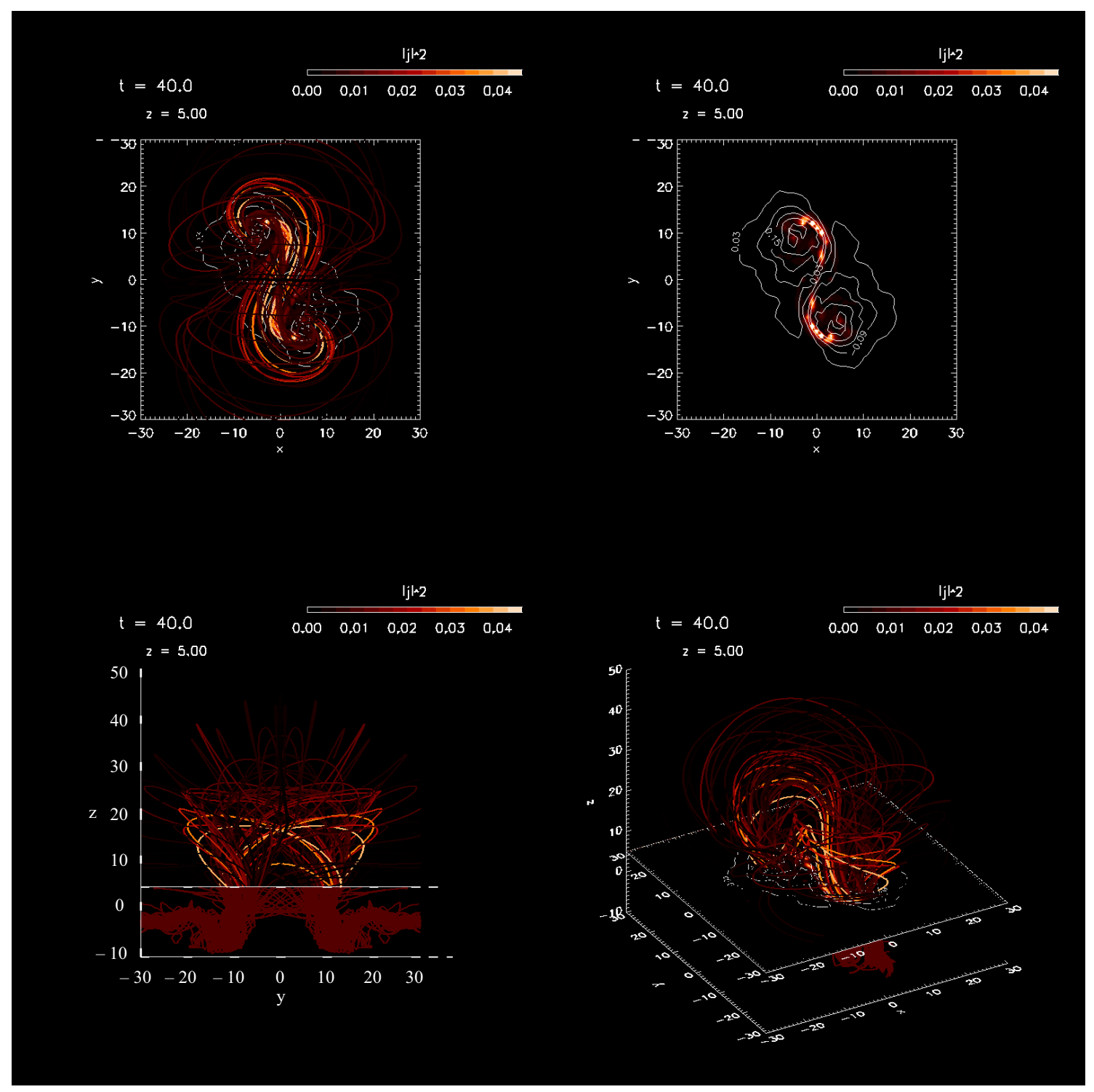

Figure 19: Double J-shaped structure formed inside an emerging flux tube. Colors of field lines represent the strength of current density measured at their chromospheric footpoints. Top, side and perspective views are presented at top-left, bottom-left, and bottom-right panels, respectively. The top-right panel shows the distribution of current density at a chromospheric plane (from Magara, 2004). 
sigmoid are located at inner central part of a magnetic structure formed through the emergence of a twisted flux tube, as we discussed in Section 3.1.2.

Regarding the geometrical feature of sigmoid, Titov and Démoulin (1999) suggests that sigmoid is composed of double J-shaped structure which is formed at the interface between an emerging flux tube and the overlying potential-like field. On the other hand, an emerging flux tube itself also produces similar double J-shaped structure inside the flux tube when it is twisted (see Figure 19, Magara, 2004). The twist of field lines is important in forming sigmoidal structure (Matsumoto et al., 1998); a potential field-like structure is formed by a weakly twisted flux tube (Figure 20, Magara, 2006). Here the illuminated field lines have a relatively high current density distributed at their chromospheric footpoints.
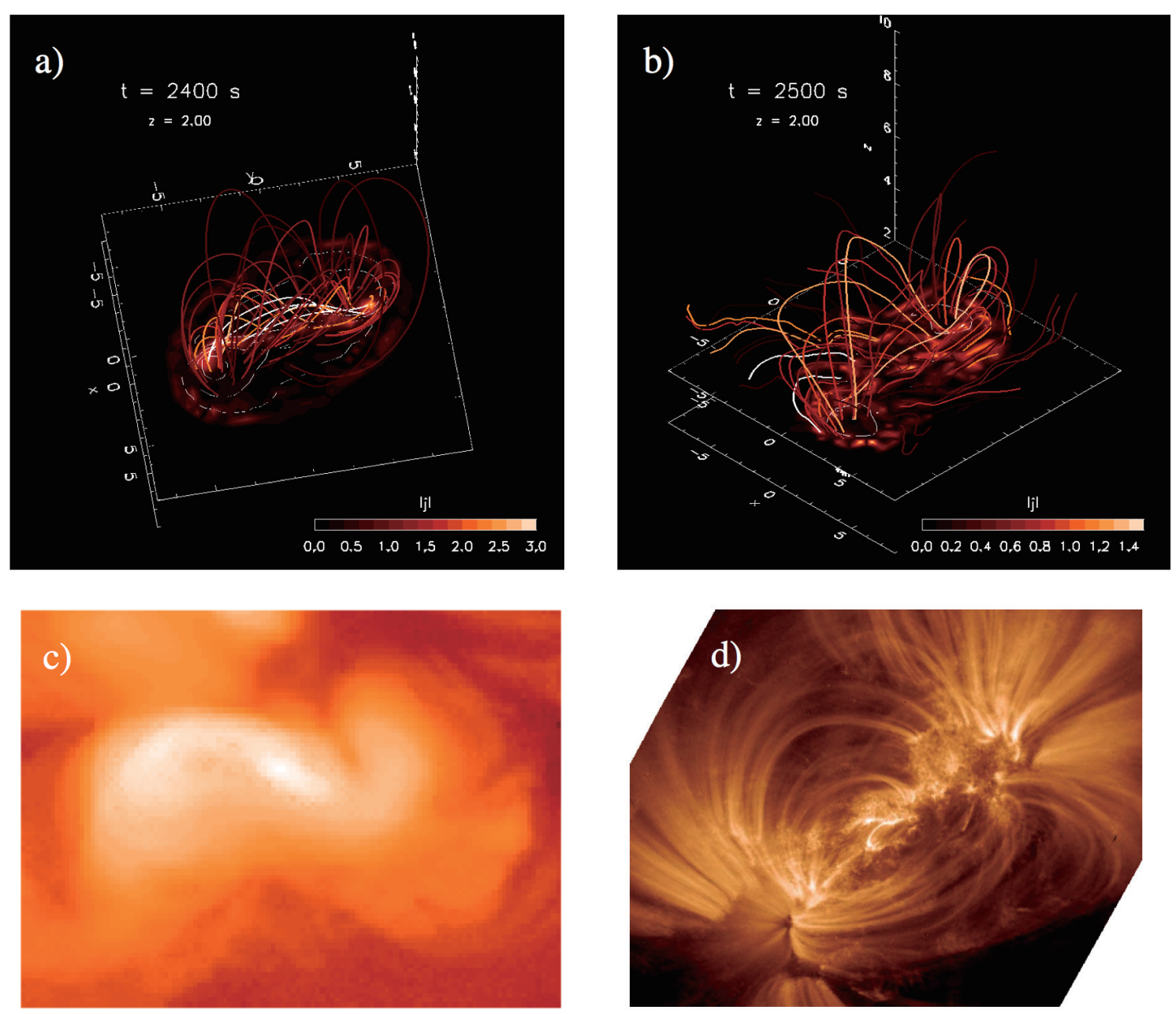

Figure 20: Magnetic structure formed by a (a) highly and (b) weakly twisted flux tube. The bottom panels (c, d) show an observed example in each case (from Magara, 2006).

The formation of sigmoid may be related to the emergence of U-loops distributed below the axis of a twisted flux tube. Magara and Longcope (2001) show that these U-loops can explain the observational chirality rule of sigmoid (Canfield et al., 1999), mentioning that inverse S-shaped (foward S-shaped) sigmoid tends to have left-handed (right-handed) twist. They also found that high current density is preferentially distributed at the chromospheric footpoints of these U-loops 
(Figure 21).

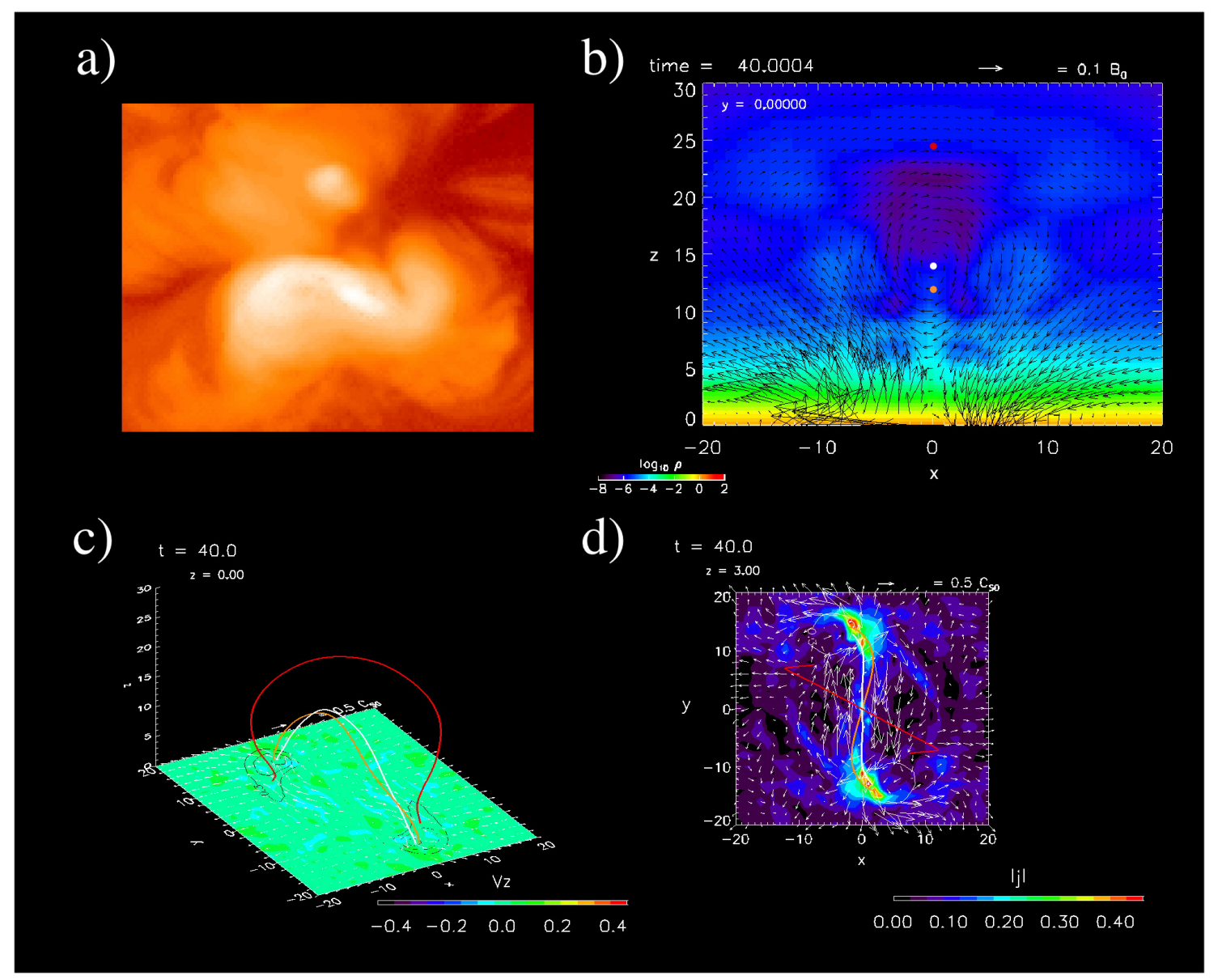

Figure 21: (a) Sigmoid observed by the soft X-ray telescope on board Yohkoh (courtesy of the Yohkoh team members). (b) Two-dimensional map of gas density (color map) and the magnetic field (black arrows) projected onto the middle plane at $y=0$, which is obtained by the emergence of a twisted flux tube (initially placed along the $y$-axis). The red, white, and orange dots represent the locations where red (above the axis), white (axis), and orange (below the axis) field lines cross this plane. (c) Threedimensional viewgraph of these magnetic field lines. The bottom map at $z=0$ shows horizontal velocity field (white arrows), vertical velocity (color map), and vertical magnetic field (contour lines). (d) Top view of (c), where a color map shows the absolute value of the current density measured at $z=3$ (chromospheric level) (from Magara and Longcope, 2001).

Figure 22 schematically explains the sigmoid formation displayed in Figure 21 (here the cross section of an emerging flux tube is shown). The upper half of the flux tube contains $\Omega$-loops which continue to expand via active magnetic buoyancy. The lower half is occupied by U-loops which tend to remain below the surface because the mass accumulates at dipped part of U-loops. However, those U-loops distributed close to the axis of the flux tube can emerge because they have a shallow dip (Magara, 2011), and they form sigmoidal structure (in this respect, since the axis becomes bent after emerging, such a shallow dip might disappear to make U-loops take an $\Omega$-shape; see Magara, 2004). Below these emerged U-loops, the magnetic field is vertically stretched and forms a current sheet in the corona, which in fact plays a key role in producing a flare, as explained in

Living Reviews in Solar Physics

http://www. livingreviews .org/lrsp-2011-6 


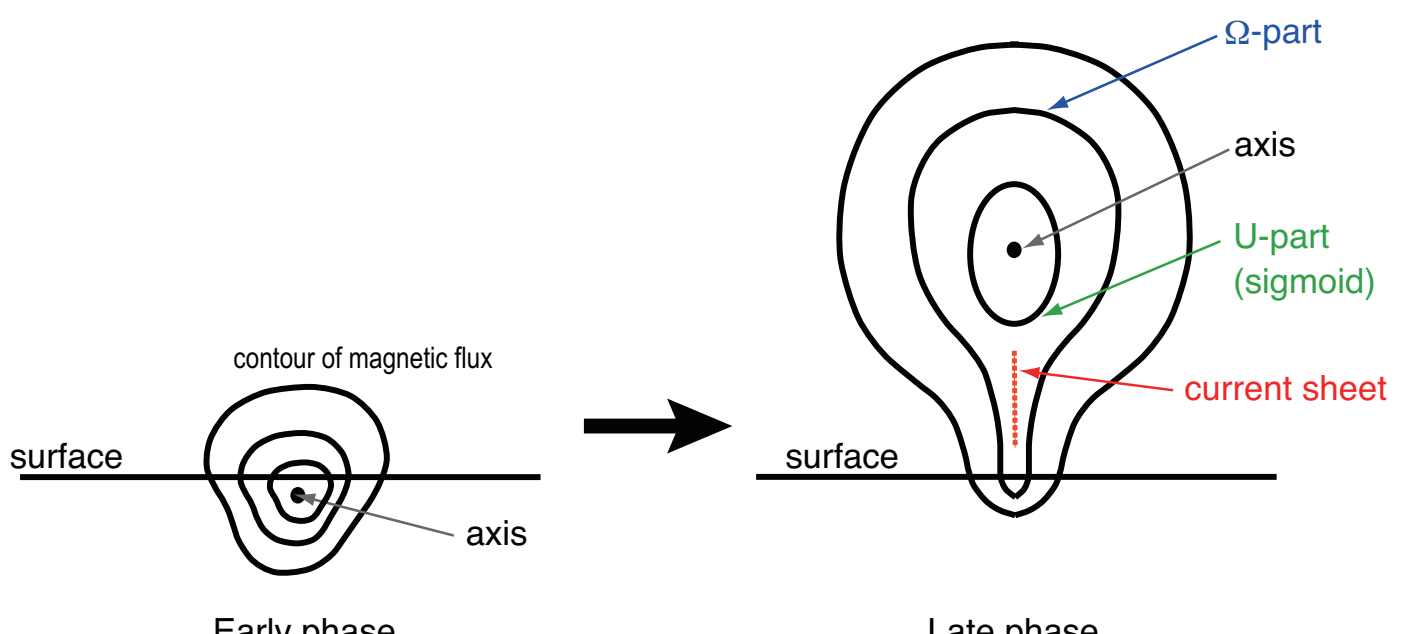

Figure 22: Sigmoid formation by an emerging flux tube. Shown is the cross section of the flux tube.

the succeeding sections. Generally, it is not easy to directly observe a magnetic structure formed in the corona, although the appearance of sigmoid might be an indicator that the axis of the flux tube already reaches the corona and a current sheet is being developed below the axis. This may explain why sigmoid is often observed as a precursor of a flare.

The three-dimensional (3D) distribution of current density inside an emerging flux tube was investigated by Manchester IV et al. (2004). Recently, the temporal development of 3D distribution of current density has been reported in Archontis et al. (2009), where a comparison between 3D distribution of current density and soft X-ray images of a sigmoid is presented. They show that the double J-shaped structure are merged into a single sigmoidal structure, just as observations show (Figure 23).

It is not clear whether sigmoid is just a thin current layer formed at the interface between two magnetic flux domains such as overlying and emerging fields, or it is more like a volumetric structure occupied by field lines with strong field-aligned current flowing on them. Three-dimensional modeling focused on the magnetic structure of an observed sigmoid such as force-free field modeling (see the next section) could provide useful information to clarify this issue. 

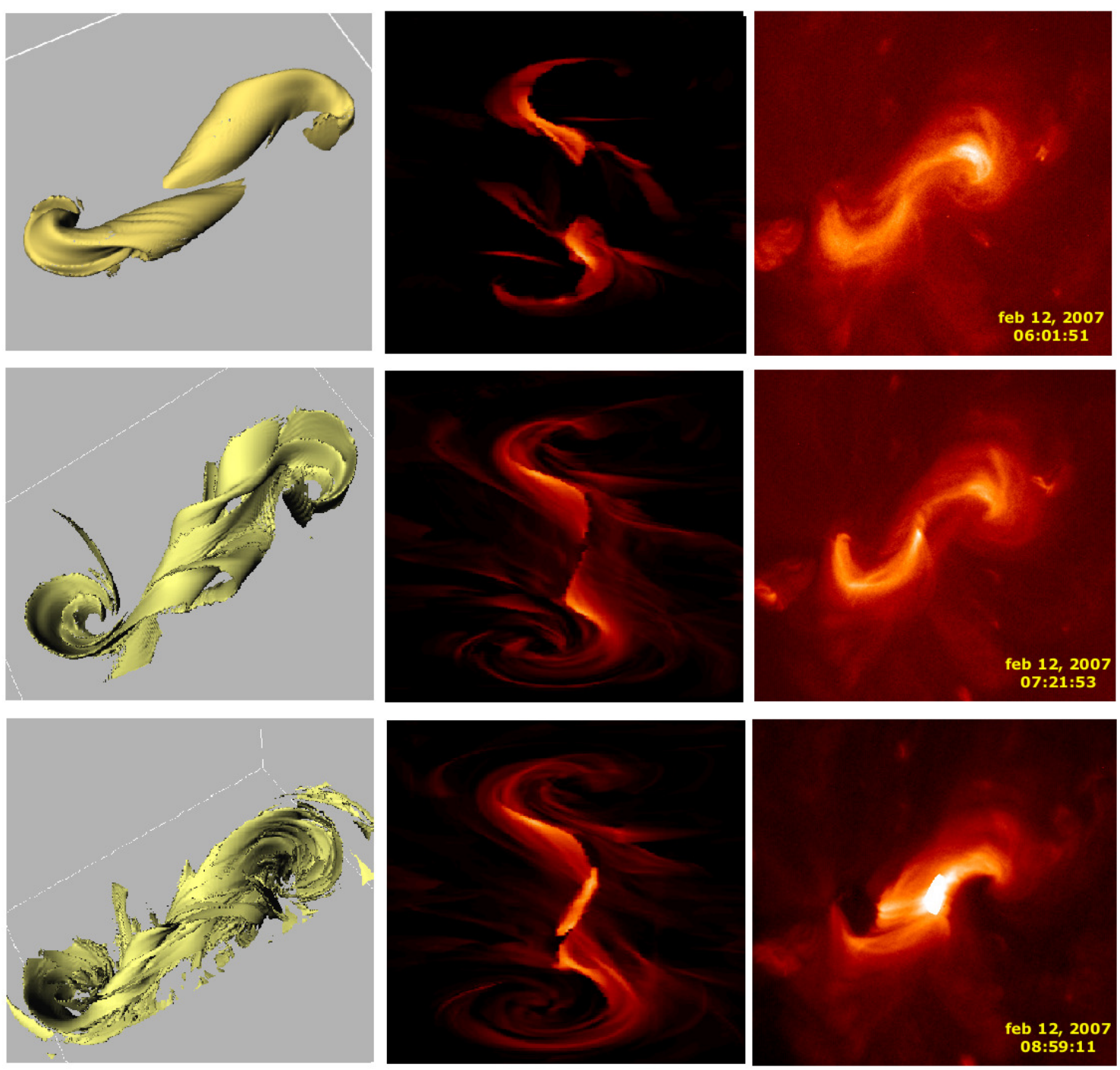

Figure 23: Comparison between simulations (left and middle columns) and soft X-ray observations (right column). The left column shows the evolution of the isosurface of current density, the middle one shows the corresponding snapshots of the heating term, and the right column shows soft X-ray images at three different times during the evolution of the sigmoidal structure (from Archontis et al., 2009). 


\subsubsection{Force-free field}

As we have explained in the previous section, the field-aligned electric current is a key factor in producing a flare, so we need the modeling that describes how the field-aligned current is distributed in a magnetic structure. In this sense, the potential-field modeling is insufficient because it cannot tell the distribution of electric current in a magnetic structure. This is provided by the force-freefield modeling where the magnetic field keeps force-balance by itself inside a magnetic structure, satisfying the following equation except at boundaries of the structure:

$$
\mathbf{j} \times \mathbf{B}=0,
$$

where $\mathbf{j}=\frac{c}{4 \pi} \nabla \times \mathbf{B}$ is the current density. Equation (10) can be transformed to $\nabla \times \mathbf{B}=\alpha \mathbf{B}$ where $\alpha$ is a scalar, and using $\nabla \cdot \mathbf{B}=0$ we can obtain $\mathbf{B} \cdot \nabla \alpha=0$, so $\alpha$ is constant along each field line. When $\alpha$ is constant all over the magnetic structure, it is called linear force-free field, otherwise it is called nonlinear force-free field. In any force-free field, the electric current always flows along field lines (volumetric field-aligned current).

Force-free field provides a method to reconstruct coronal magnetic field from surface magnetic field, the latter of which is easier to observe than the former. By using this method, Nakagawa et al. (1971) studied the magnetic structure of an isolated sunspot. Similarly, Sheeley Jr and Harvey (1975) construct a magnetic configuration formed by discrete flux sources at the surface. The helical nature of force-free field has been investigated by Sakurai (1979). By considering a series of force-free states, Barnes and Sturrock (1972), Low and Nakagawa (1975), and Low (1977) investigated the characteristics of evolving magnetic structure.

Recent developments are found in the attempt to combine force-free field modeling and the observation of surface magnetic field. Wheatland et al. (2000) introduce an optimization method to calculate force-free field, and according to this method Wiegelmann et al. (2000) calculate a forcefree field based on observed surface magnetic field. Wiegelmann and Neukirch (2006) extended this method to calculate a magnetohydrostatic state. Régnier and Priest (2007) estimated difference among energy states of nonlinear force-free, linear force-free, and potential field in several active regions. A good review on various methods to calculate force-free field is given by Wiegelmann (2008). Very recently, Valori et al. (2010) have done two important steps in coronal field modeling. First, they show that it is possible to compute the coronal field even when it is significantly twisted (more than one turn), which was not obvious from previous studies. Second, they relate the specific behavior of the extrapolated field to its MHD evolution when the test field is out of equilibrium.

\subsubsection{Aly-Sturrock conjecture}

Let us mention a famous conjecture on the energy state of force-free field. Barnes and Sturrock (1972) calculated a series of force-free fields in cartesian coordinates, showing that the energy state of these force-free fields could be greater than that of the open field with the same vertical magnetic flux at the surface. However, Aly (1984) presents a conjecture that the energy of any force-free fields might be smaller than that of the open field. Yang et al. (1986) reconsidered this problem, showing that the Aly's conjecture seems to be right, which later has also been supported by Sturrock (1991). The conjecture is now known as the Aly-Sturrock conjecture on the energy state of force-free field, mentioning that the fully open-field state associated with a current sheet of infinite length and infinitesimal thickness can be reached only asymptotically and the energy of this fully open field cannot be exceeded by any other force-free fields. This in fact caused difficulty in creating a force-free state during a preflare phase, which should be reduced to the fully open-field state in a postflare phase. There have, however, been proposed various ways to avoid this difficulty, as listed below:

- Full opening of field lines is not required (creating a current sheet of finite length) (Aly, 1991). 
- Resistive processes introduce a new factor that the conjecture does not assume, that is, the resistivity allows the formation of a twisted flux tube in a highly sheared arcade. This process prevents from forming a long current sheet by increasing the magnetic energy monotonically during a preflare state. At some point of preflare phase, the flux rope becomes unstable and is launched away, creating a current sheet of finite length below an ejecting flux rope to produce a flare. Then, a CME-like event occurs without opening fully bipolar field (arcade). This actually describes "Full opening of field lines is not required" (Mikić and Linker, 1994).

- Partially open configurations are possible after the onset of a flare (Sturrock, 1991; Low, 1996).

- Cylindrically axisymmetric force-free field formed on a spherical surface gives a configuration whose energy state is not bounded by the energy limit suggested by the conjecture (LyndenBell and Boily, 1994).

- Multi-polar configuration can be free from the energy limit suggested by the conjecture (e.g., Biskamp and Welter, 1989; Antiochos et al., 1999a; Choe and Cheng, 2002).

- Non-force-free field effects (e.g., gas pressure and gravitational force) unbound the energy limit suggested by the conjecture (Sturrock, 1991).

Regarding the energetics of force-free fields, Kusano et al. (1995) demonstrate a difference between two energy states of linear force-free fields that have the same magnetic helicity (see Section 3.3). They present an energy-bifurcation theory in which a vertically elongated magnetic arcade tends to be reduced to a state in which a twisted flux rope exists.

\subsection{Magnetic helicity}

As we have seen, the topology of magnetic field is one of the key factors in determining the dynamical nature of a magnetic structure. A quantity describing how much a magnetic structure is twisted, sheared and braided is given by the magnetic helicity, which is originally defined as

$$
H_{M}(t)=\int_{z \geq 0} \mathbf{A}(t) \cdot \mathbf{B}(t) d V
$$

where $\mathbf{B}$ is the magnetic field and $\mathbf{A}$ is the vector potential for $\mathbf{B}$. In a semi-infinite region above the surface where the cartesian coordinates $(x, y, z)$ is used ( $z$-axis is directed upward and $z=0$ is the surface), the magnetic helicity $H_{M}$ defined by Equation (11) becomes gauge-invariant and is called relative magnetic helicity (Berger and Field, 1984; Finn and Antonsen Jr, 1985) if the vector potential is given by DeVore (2000)

$$
\mathbf{A}(x, y, z, t)=\mathbf{A}_{C}(x, y, 0, t)-\hat{z} \times \int_{0}^{z} \mathbf{B}\left(x, y, z^{\prime}, t\right) d z^{\prime}
$$

Here $\mathbf{A}_{C}$ is the vector potential for the potential field that has the same vertical magnetic flux at the surface as $\mathbf{B}$. The exact form of $\mathbf{A}_{C}$ is

$$
\mathbf{A}_{C}(x, y, z, t)=\nabla \times \hat{z} \int_{z}^{\infty} \phi_{C}\left(x, y, z^{\prime}, t\right) d z^{\prime}
$$

where $\phi_{C}$ is the scalar potential for the potential field, given by 


$$
\phi_{C}(x, y, z, t)=\frac{1}{2 \pi} \int_{z=0} \frac{B_{z}\left(x^{\prime}, y^{\prime}, 0, t\right) d x^{\prime} d y^{\prime}}{\left[\left(x-x^{\prime}\right)^{2}+\left(y-y^{\prime}\right)^{2}+z^{2}\right]^{1 / 2}} .
$$

Here the potential field is also defined in the semi-infinite space $(z \geq 0)$. In any numerical simulations, the domain of simulation is finite, surrounded by boundaries. To compromise this unavoidable discrepancy, simulations are terminated before the emerging magnetic field reaches the boundaries of the domain so that the emerging field feels that the domain is infinite (still, there are effects of waves and flows reflected by boundaries on emerging field).

The relative magnetic helicity quantitatively describes how much a magnetic structure is sheared compared to a (shearless) potential field. In a right-handed coordinate system the relative magnetic helicity takes a positive (negative) value when a magnetic structure is sheared in a right-handed (left-handed) way, while it take zero in the case of a potential field. Hereafter, we simply use the magnetic helicity to express the relative magnetic helicity.

The helicity flux across the surface is given by

$$
F_{H z}=-2 \int_{z=0}\left(A_{x} v_{x}+A_{y} v_{y}\right) B_{z} d x d y+2 \int_{z=0}\left(A_{x} B_{x}+A_{y} B_{y}\right) v_{z} d x d y
$$

where the first term on the right hand side of Equation (15) represents the shear/twist by horizontal motions and will be called the shear term. The second term, called the emergence term, would clearly vanish in the absence of vertical flows.

Figure 24 shows a result on the injection of magnetic flux, energy and helicity via the emergence of a twisted flux tube. Since the flux tube has left-handed twist, negative magnetic helicity is injected during the emergence of the flux tube. This figure indicates that the injection of magnetic energy and helicity is carried out mainly by the emergence term during the early phase of emergence, while it is done by the shear term during the late phase when the emergence of magnetic field almost terminates (see the time variation of emerged magnetic flux at the top right of the figure). In some case, the emergence term gives net loss of magnetic energy and helicity in the late phase of emergence when a strong downflow arises along emerged loops, dragging magnetic field downward below the surface and causing the net loss (Magara, 2008). Another important result from Figure 24 is that the injection of magnetic flux, energy, and helicity has already saturated before the inner central part of the flux tube expands into the corona and make a well developed magnetic structure. This suggests that the strong energy injection at the surface may not be directly related to the activation of a magnetic structure formed on the Sun; the activation may depend on the magnetic configuration developed in the corona.

We have discussed the injection of magnetic helicity via the emergence of an integrated flux tube, while the emergence of magnetic field sometimes proceeds in the form of a partially split flux tube, as illustrates in Figure 25a (Zwaan, 1985). The dynamic process and associated helicity injection caused by a partially split flux tube were numerically simulated in Magara (2008) where a flux tube composed of two splitting portions forms a quadrupolar-like distribution at the surface. In this simulation the mutual magnetic helicity (Priest and Forbes, 2000) arises when these two portions emerge (Figures 25b-d), and the sign of mutual helicity is opposite to the sign of self helicity contained by those individual portions. We cannot say that this is a general result although the opposite sign of helicity is also observed in real active regions where multiple flux tubes successively emerged (Lim and Chae, 2009; Park et al., 2010). This suggests that these multiple flux tubes might correspond to the splitting portions of a global flux tube, forming multiple flux domains on the surface and a flare is expected to occur at the interface between different flux domains (see Sections 4.2 and 4.3). 
a)
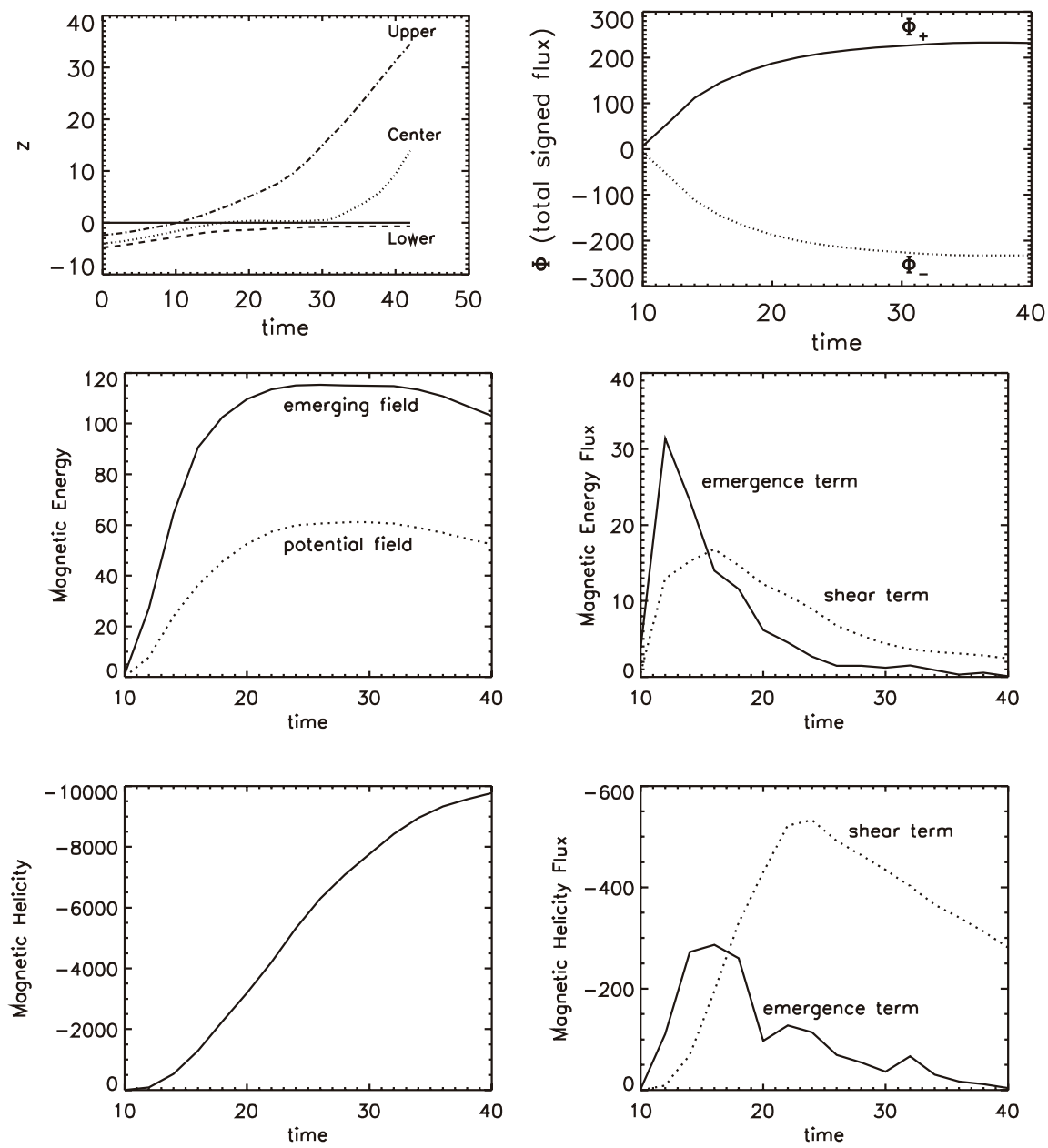

b)
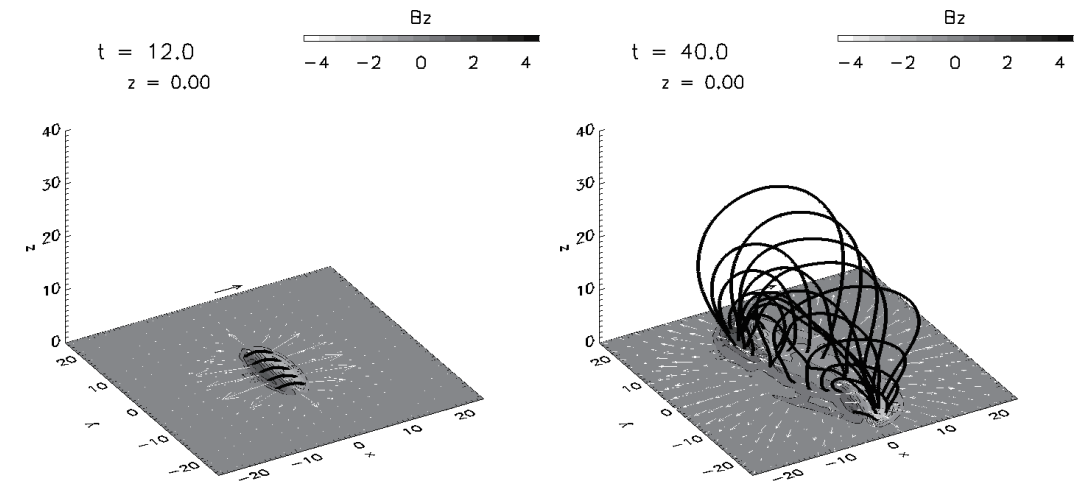

Figure 24: (a) Top-left panel: Temporal development of the height of three field lines which compose a twisted flux tube of left handedness (contains negative magnetic helicity). Top-right panel: Time variation of emerged magnetic flux. Middle-left panel: Time variation of magnetic energy injected to the atmosphere $(z>0)$. Middle-right panel: Time variation of magnetic energy flux. Bottom-left panel: Time variation of magnetic helicity injected to the atmosphere. Bottom-right panel: Time variation of magnetic helicity flux. (b) Snapshot of the emerging flux tube at the early $(t=12$, left panel $)$ and late $(t=40$, right panel $)$ phases (from Magara and Longcope, 2003). 
a)

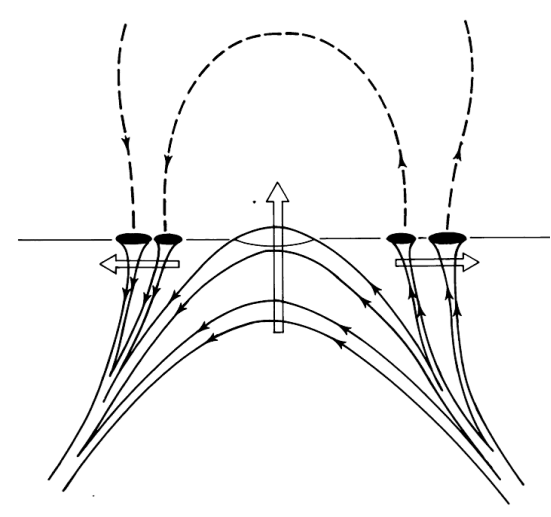

c)

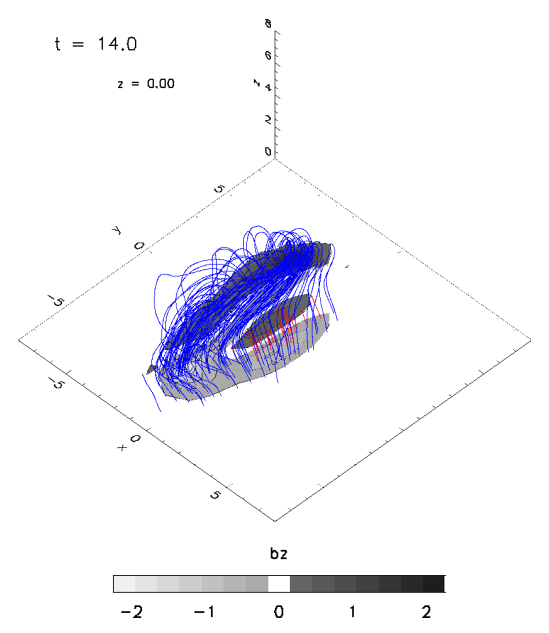

b)

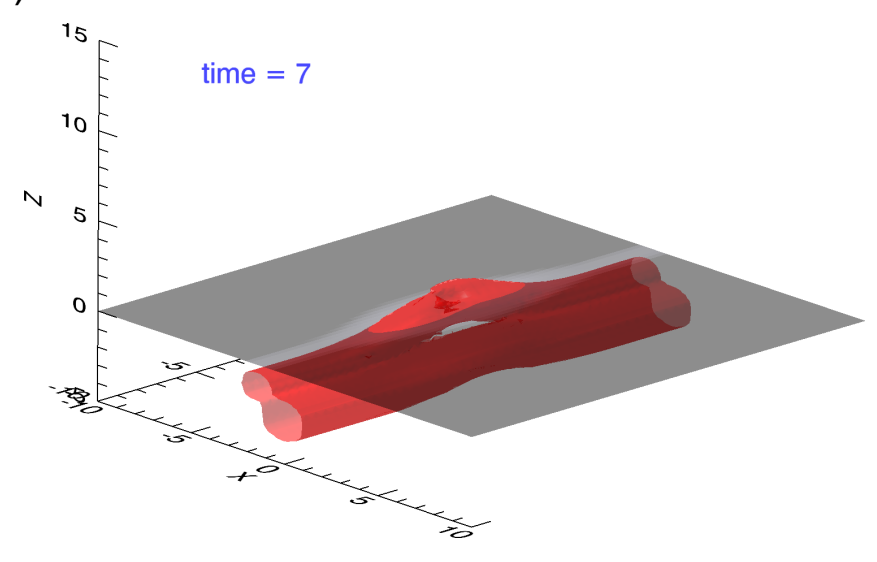

d)

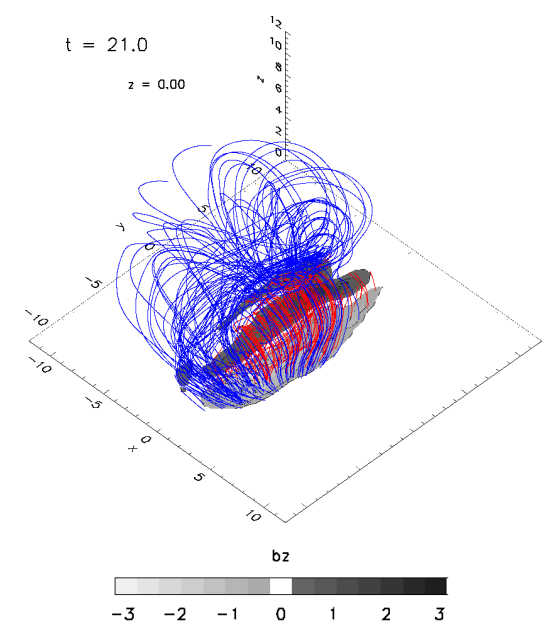

Figure 25: (a) Emergence of a partially split flux tube (from Zwaan, 1985). (b) Simulation of the emergence of a partially flux tube. (c) - (d) Quadrupolar-like structure formed by this flux tube. Panels (b) - (d) from Magara (2008). 
Deriving observationally how much magnetic helicity is injected into the atmosphere has widely been done, which is a key to the understanding of the relationship between helicity evolution and the occurrence of active phenomena including flares (Chae, 2001; Moon et al., 2002; Nindos and Zhang, 2002; Kusano et al., 2002; Démoulin and Berger, 2003; Yang et al., 2004; Pariat et al., 2005; Jeong and Chae, 2007; Magara and Tsuneta, 2008). These studies are important in that we can derive the characteristics of helicity evolution and use it to predict the occurrence of active phenomena on the Sun. A recent review by Démoulin and Pariat (2009) is worth reading for those who are interested in this subject. 


\section{Energy Release}

We here focus on magnetic reconnection, the central engine that enables the rapid release of the magnetic energy accumulated via the energy build-up process mentioned in the previous section. We first explain the basic physics of magnetic reconnection, and then discuss theoretical models which demonstrate how magnetic reconnection works to produce flares and flare-associated phenomena.

\subsection{Magnetic reconnection}

A finite value of resistivity causes the so-called Ohmic dissipation of electric current (cross-field current), which is especially efficient in a region where an intensive electric current flows, called current sheet. When the dissipation causes the topological change of magnetic field, the magnetic field is then reduced to a state that has lower energy than before. This is called magnetic reconnection. Through this process the magnetic energy stored inside and outside a current sheet is converted to kinetic and thermal energy. Magnetic reconnection is also accompanied by the generation of strong electric field around a current sheet (convective electric field), which could accelerate charged particles. The concept of magnetic reconnection is simple, although its physics is deep and wide, so we here suggest one of the textbooks that provide a comprehensive explanation of this really complicated process (Priest and Forbes, 2000). In the following, we briefly explain the basics of magnetic reconnection, focusing on its time scale in the environment of the solar corona.

\subsubsection{Basic models of magnetic reconnection}

Figure 26 shows a current sheet where magnetic reconnection occurs. The speed of reconnection (reconnection rate) is measured as the magnetic flux reconnecting per unit time, $d \Phi / d t$. Here, $\Phi$ is the magnetic flux per unit length in the direction perpendicular to the plane containing the current sheet. For a steady state, the Faraday's equation and Ohm's law give the reconnection rate as follows:

$$
\frac{d \Phi}{d t}=c E=4 \pi c^{-1} \eta j=v_{i} B_{i}=\text { constant } .
$$

Here $E$ and $j$ are the electric field and current density in a diffusion region with finite magnetic diffusivity $\eta$, and in a steady state we have $\nabla \times \mathbf{E}=\mathbf{0}$, implying that $c \mathbf{E}=-\mathbf{v}_{i} \times \mathbf{B}_{i}$ outside the diffusion region (inflow region) with zero diffusivity is equal to $\mathbf{E}=4 \pi c^{-2} \eta \mathbf{j}$ in the diffusion region. Also $v_{i}$ is the inflow speed of plasma entering the diffusion region and $B_{i}$ is the magnetic field, both of which are measured in the inflow region. In deriving Equation (16), we assume that the diffusivity takes nonzero value only in the diffusion region.

The reconnection rate given by Equation (16) can be non-dimensionalized using the Alfvén Mach number $M_{A}$ in the inflow region;

$$
\frac{d \Phi / d t}{v_{A} B_{i}}=\frac{v_{i} B_{i}}{v_{A} B_{i}}=v_{i} / v_{A}=M_{A},
$$

where $v_{A}$ is the Alfvén velocity measured in the inflow region. We then define the reconnection time in a current sheet of the length $L$ :

$$
t_{\mathrm{rec}}=L / v_{i}=t_{A} / M_{A}
$$

where $t_{A}=L / v_{A}$ is the Alfvén transit time. When we take $L$ as the typical length of coronal magnetic structure $\left(10^{4}-10^{5} \mathrm{~km}\right), t_{A}$ is something between 10 and $100 \mathrm{~s}$. 


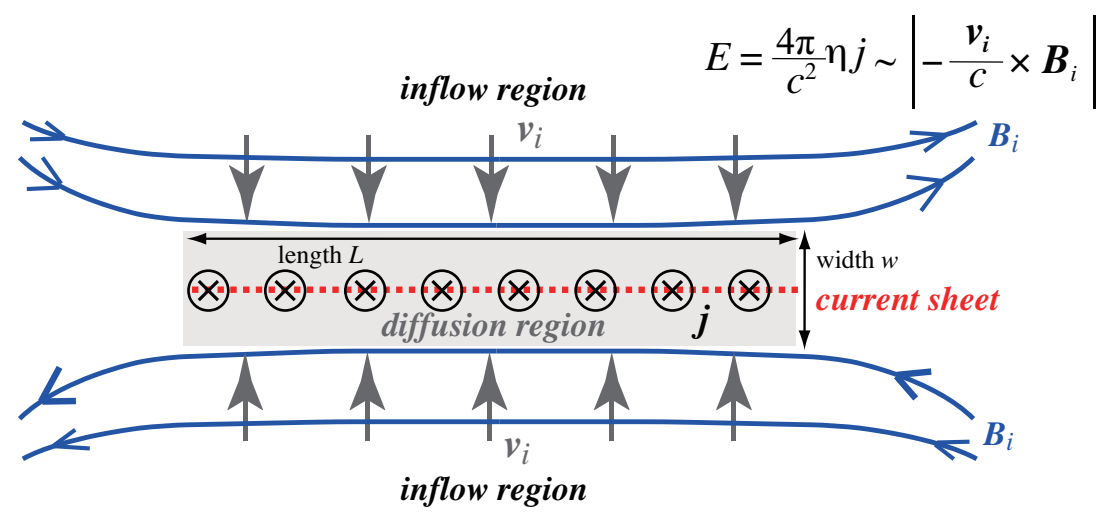

Figure 26: Magnetic reconnection in a current sheet.

\subsubsection{Sweet-Parker model}

In the Sweet-Parker model for magnetic reconnection (Sweet, 1958; Parker, 1957), the nondimensionalized reconnection rate is given by

$$
M_{A} \simeq R_{m}^{-1 / 2}
$$

where

$$
R_{m}=v_{A} L / \eta
$$

is called the Lundquist number (magnetic Reynolds number with the typical velocity $v_{A}$ ). $R_{m}$ typically takes a value of $10^{14}$ in the corona, so $M_{A} \sim 10^{-7}$. This gives $t_{\mathrm{rec}} \sim 10^{8}-10^{9} \mathrm{~s}$ from Equation (18), which is much longer than the typical time scale of a flare $\left(10^{2}-10^{4} \mathrm{~s}\right)$. Hence, this model cannot explain rapid energy release in a flare (Parker, 1963).

\subsubsection{Petschek model}

Petschek proposed another steady model for magnetic reconnection that enables faster energy release than the Sweet-Parker model. The reconnection rate in the Petschek model is given by

$$
M_{A} \simeq \frac{\pi}{8 \ln R_{m}},
$$

which is $0.01-0.1$ in the corona (Petschek, 1964). This value gives a reconnection time comparable to the time scale of a flare. Forbes and Priest (1987) later extended the model proposed by Petschek. However, the evolution to the steady state assumed in the Petschek's model has not been fully understood yet, and the controlling factor of the reconnection rate is still a controversial issue (Biskamp, 1993; Priest and Forbes, 2000). In this respect, Forbes and Priest (1987) did an extensive investigation into the system where two-dimensional steady reconnection is operated. They show that the speed of magnetic reconnection is controlled by the spatial pattern of flow in the inflow region. They have demonstrated that a diverging pattern of inflow produces a flux pileup configuration around the diffusion region, while a converging pattern of inflow gives a magnetic configuration that is similar to the one suggested by the Petschek model.

In order to derive the reconnection rate from observations (Isobe et al., 2002; Nagashima and Yokoyama, 2006), it is crucial to obtain the inflow speed of plasma around a current sheet (Yokoyama et al., 2001; Narukage and Shibata, 2006; Hara and Ichimoto, 1997; Hara et al., 2006; Miklenic et al., 2009, and references therein). Suppose that the reconnection rate is $M_{A} \sim$ 
$0.01-0.1$, which is suggested by the Petschek model, the inflow speed would be $10-100 \mathrm{~km} \mathrm{~s}^{-1}$ because the Alfvén velocity in the corona is estimated as

$$
v_{A}=B /(4 \pi \rho)^{1 / 2} \sim 1000\left(\frac{B}{50 \mathrm{G}}\right)\left(\frac{n}{10^{10} \mathrm{~cm}^{-3}}\right)^{-1 / 2} \mathrm{~km} \mathrm{~s}^{-1} .
$$

In this case, the energy-release rate due to magnetic reconnection is given by

$$
\begin{aligned}
\frac{d E}{d t} & =L^{2} B_{i}^{2} v_{i} / 4 \pi \\
& \sim 4 \times 10^{28}\left(\frac{v_{i}}{100 \mathrm{~km} \mathrm{~s}^{-1}}\right)\left(\frac{B_{i}}{100 \mathrm{G}}\right)^{2}\left(\frac{L}{2 \times 10^{9} \mathrm{~cm}}\right)^{2} \mathrm{erg} / \mathrm{s},
\end{aligned}
$$

where we assumed that a diffusion region has the size of $L$ in both length and depth. $10^{28} \mathrm{erg} / \mathrm{s}$ is a typical value of energy-release rate in the impulsive phase of a flare (e.g., Masuda et al., 1994).

\subsubsection{Locally enhanced resistivity}

The evolution leading to the steady state assumed in the Petschek model has widely been investigated with the help of numerical simulations. Here we discuss two types of reconnection: (i) driven-type reconnection (Sato and Hayashi, 1979), and (ii) spontaneous-type reconnection (Ugai and Tsuda, 1977). In the driven-type reconnection, fast reconnection is achieved by an external object that drives an inflow toward a current sheet. Sato and Hayashi (1979) performed two-dimensional MHD simulations in which an inflow is driven as boundary condition, showing that the Petschek-type reconnection occurs when the resistivity is locally enhanced in a current sheet. Biskamp (1986) also followed the concept of the driven-type reconnection, but the result shows that when a uniform resistivity is assumed the Petschek-type reconnection does not arise, instead the Sweet-Parker-type reconnection occurs.

For the spontaneous-type reconnection, resistivity is locally enhanced inside a current sheet via some microscale instabilities attributed to the nature of a current sheet, then an inflow spontaneously arises without any external sources, which draws plasma toward a current sheet. In this case the nature of a current sheet is the primary factor causing fast reconnection. Ugai and Tsuda (1977) performed two-dimensional MHD simulations of the spontaneous-type reconnection, and successfully reproduced the Petschek-type reconnection by locally enhancing resistivity inside a current sheet. Their result was later confirmed by Scholer (1989) showing that fast reconnection is closely related to the local enhancement of resistivity. Yokoyama and Shibata (1994) presented a result on the role of locally enhanced resistivity in the Petschek-type reconnection.

In either driven or spontaneous case, the local enhancement of resistivity seems an essential process, by which the Petschek-type reconnection occurs (Kulsrud, 2001; Uzdensky and Kulsrud, 2000). Also there is an issue about the origin of the external source assumed in the driven-type reconnection. This is crucial when we apply the driven-type reconnection to space plasma because we should identify the object that drives an inflow in a free space occupied by space plasma. In the case of laboratory plasma, we may easily identify such external objects outside a current sheet.

Very recently, it was shown that the classical Petschek-type solution with fast stationary magnetic reconnection is possible with a spatially uniform resistivity (Baty et al., 2009a,b) when a non-uniform viscosity distribution is assumed, which gives a new insight into the relation between the distribution of resistivity and the speed of reconnection. Also the self-similarity aspect of Petschek-type reconnection has been investigated (Nitta, 2010, and references therein).

\subsubsection{Tearing instability and fractal reconnection}

Following the discussion presented above, we then focus on how the resistivity is locally enhanced in a current sheet. Firstly, it should be noticed that even if the resistivity is uniformly distributed 


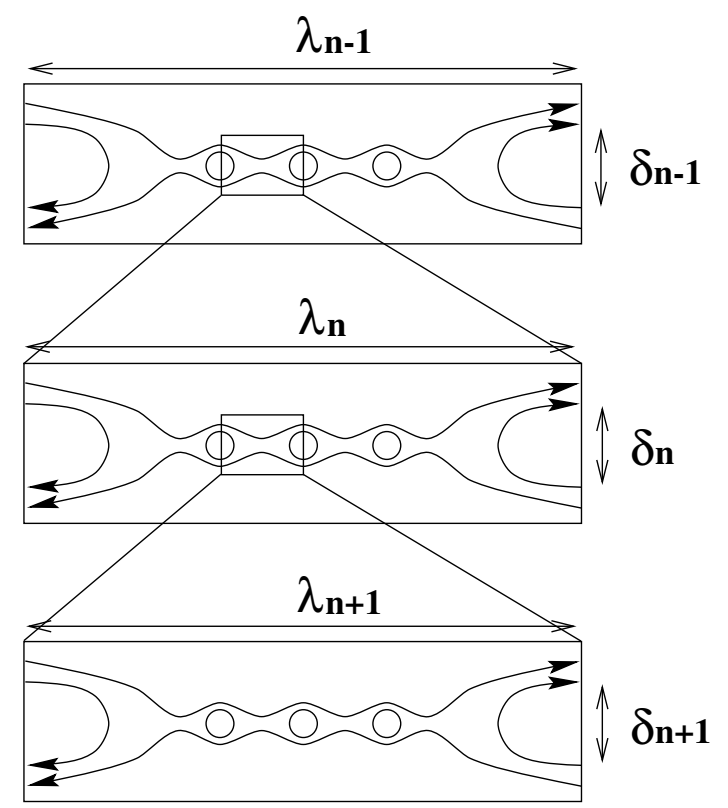

Figure 27: Fractal current sheet (from Shibata and Tanuma, 2001).

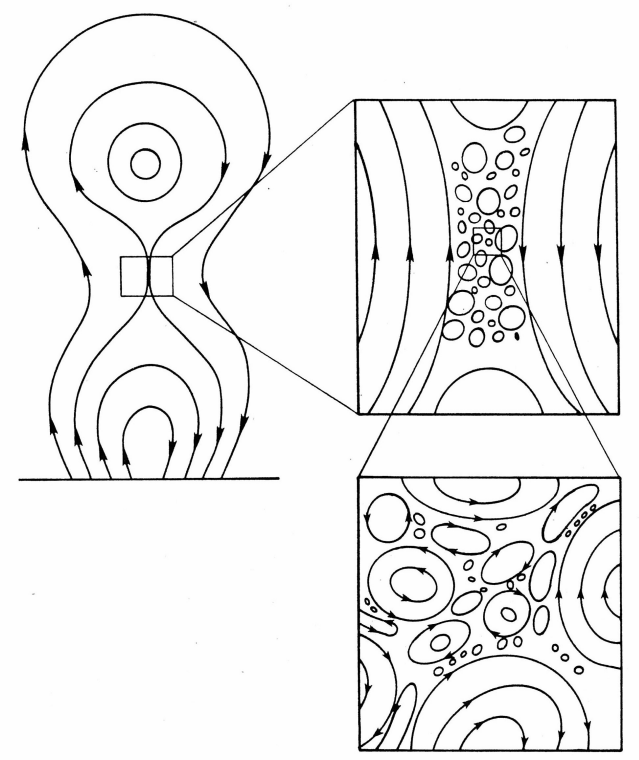

Figure 28: Fractal current sheet in a flare (from Tajima and Shibata, 1997). 
in a current sheet, the sheet is subject to the tearing instability (Furth et al., 1963; Steinolfson and van Hoven, 1984; Horton and Tajima, 1988), which eventually introduces nonuniformity into the current sheet where a series of magnetic islands are formed.

These magnetic islands tend to coalesce together to make a large magnetic island, and during this coalescencing process the magnetic energy is efficiently converted to kinetic energy (Bhattacharjee et al., 1983; Sakai and Ohsawa, 1987; Tajima et al., 1987). Nonsteady reconnection associated with multiple magnetic islands (Choe and Cheng, 2000) often causes impulsive bursty reconnection (Priest, 1985). Recently, Karlický and Bárta (2007) and Bárta et al. (2008) performed a series of simulations where tearing process is incorporated into the evolution of a flare.

In a developed phase of this process, the thickness of a current sheet is locally reduced so as to produce an extremely thin layer (Magara and Shibata, 1999). Tanuma et al. (2001) investigated this thinning process in detail by performing two-dimensional MHD simulations, which reproduces the long-term evolution of a current sheet subject to the tearing instability. They have shown that secondary tearing instability occurs in an extremely thin layer. It is suggested that successive tearing processes might produce a hierarchy of multi-scale magnetic islands coexisting in a current sheet, which becomes a fractal current sheet (Shibata and Tanuma, 2001; Nishizuka et al., 2009), as shown in Figures 27 and 28.

If the thickness of a current sheet is reduced to microscales such as the ion's Larmor radius or ion's inertial length, kinetic processes such as the decoupling of ions and electrons as well as the interaction of waves and charged particles become important. These kinetic processes might enhance the resistivity significantly over the Spitzer resistivity based on collisions among particles (Spitzer, 1962). The coupling between macroscopic (MHD) and microscopic (kinetic) processes is important during magnetic reconnection, which has intensively been investigated by theory, simulation, and laboratory (Treumann and Baumjohann, 1997; Biskamp et al., 1995; Horiuchi and Sato, 1999; Yamada et al., 2000; Birn et al., 2001; Shay et al., 2001; Bhattacharjee et al., 2003; Drake et al., 2003; Hanasz and Lesch, 2003; Heitsch and Zweibel, 2003; Rogers et al., 2003; Ji et al., 2004; Craig and Watson, 2005).

\subsubsection{Plasmoid-induced reconnection}

It has been speculated that the speed of magnetic reconnection is related to the behavior of magnetic island, i.e., plasmoid formed in a current sheet (Shibata, 1999). While staying in a current sheet, a plasmoid significantly reduces the speed of reconnection by inhibiting an inflow accompanied by magnetic flux from entering a current sheet. When a plasmoid moves out of a current sheet, substantial amount of magnetic flux can come into the sheet, thereby triggering magnetic reconnection. This facilitates the ejection of a plasmoid via strong reconnection outflow (reconnection jet), which in turn enables new magnetic flux to continuously enter the current sheet. The positive feedback between plasmoid ejection and inflow enhancement contributes to producing fast reconnection, and eventually a plasmoid is ejected at about the Alfvén velocity. The whole process is named as plasmoid-induced reconnection by Shibata (1999). Recently, a detailed relation between plasmoid velocity and reconnection rate has been investigated by performing a series of numerical simulations (Nishida et al., 2009).

\subsection{Current-sheet formation}

The release of free energy contained in a magnetic structure is triggered by fast magnetic reconnection in a current sheet distributed in the corona, so an important question is how a current sheet is formed in the corona. Possible scenarios are:

(1) a current sheet is formed at the interface between different flux domains interacting each other. Multiple flux domains might be formed via the emergence of a partially split flux tube (Figure 25). 
(2) a current sheet is formed inside a single flux domain where sheared magnetic field develops (development of magnetic shear)

\subsubsection{Interaction of flux domains}

A simple model for interacting flux domains is given by the emerging magnetic field that touches the preexisting magnetic field (Rust, 1972; Heyvaerts et al., 1977). Figure 29 illustrates this model in which a current sheet is formed at the interface between these two magnetic domains, leading to magnetic reconnection there.
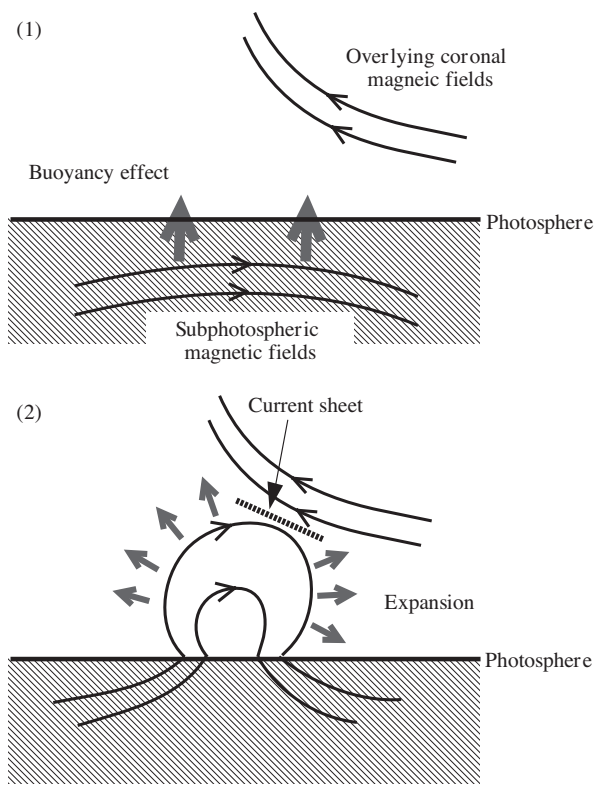

(3)

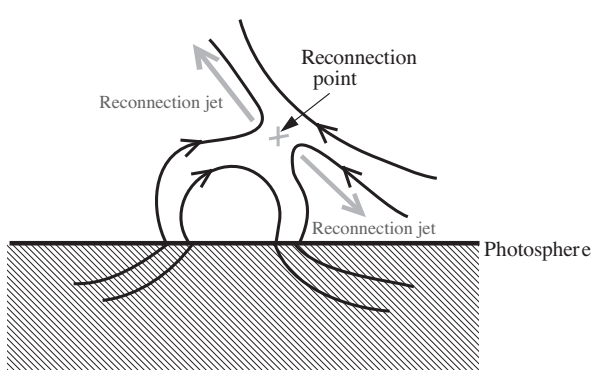

Figure 29: Formation of a current sheet via the interaction of emerging and preexisting magnetic fields.

The dynamic process of interacting flux domains has been studied extensively using numerical simulations (Forbes and Priest, 1984; Shibata et al., 1989b, 1992b; Yokoyama and Shibata, 1994, 1995, 1996). Figure 30 shows a result from a two-dimensional MHD simulation where emerging bipolar field interacts with the preexisting oblique field (Yokoyama and Shibata, 1996). This simulation clearly demonstrates how a current sheet develops via the interaction of flux domains, followed by fast magnetic reconnection producing a high-speed, collimated plasma flow (i.e., 'jet').

Recently, numerical simulations analyze the interaction of flux domains in various three-dimensional configurations, revealing more complicated dynamic processes than in two-dimensional 

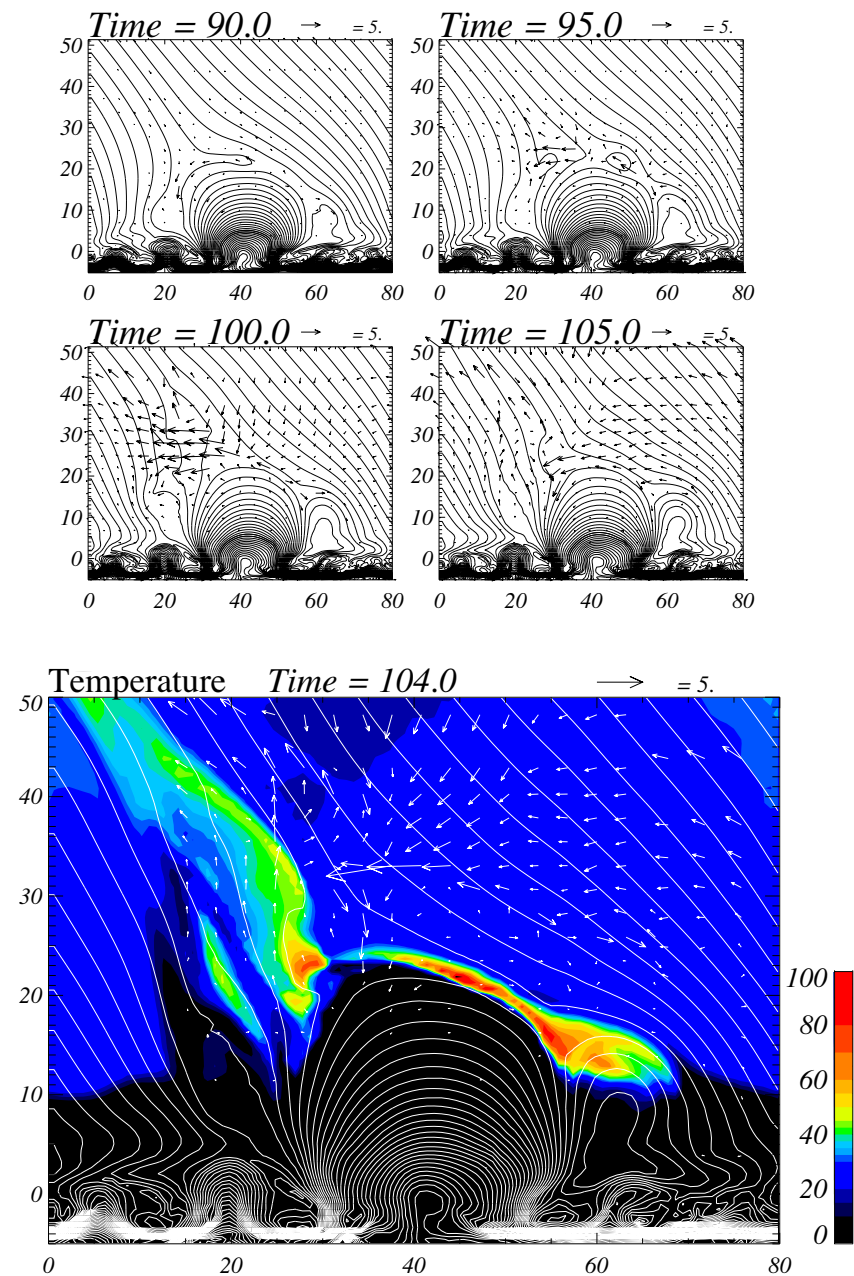

Figure 30: Interaction of emerging and preexisting fields and resultant dynamic processes, reproduced by a two-dimensional MHD simulation. The upper panel shows the evolution of magnetic field (contours) and flow (velocity field), while the lower represents the enhancement of temperature (colors) (from Yokoyama and Shibata, 1996). 

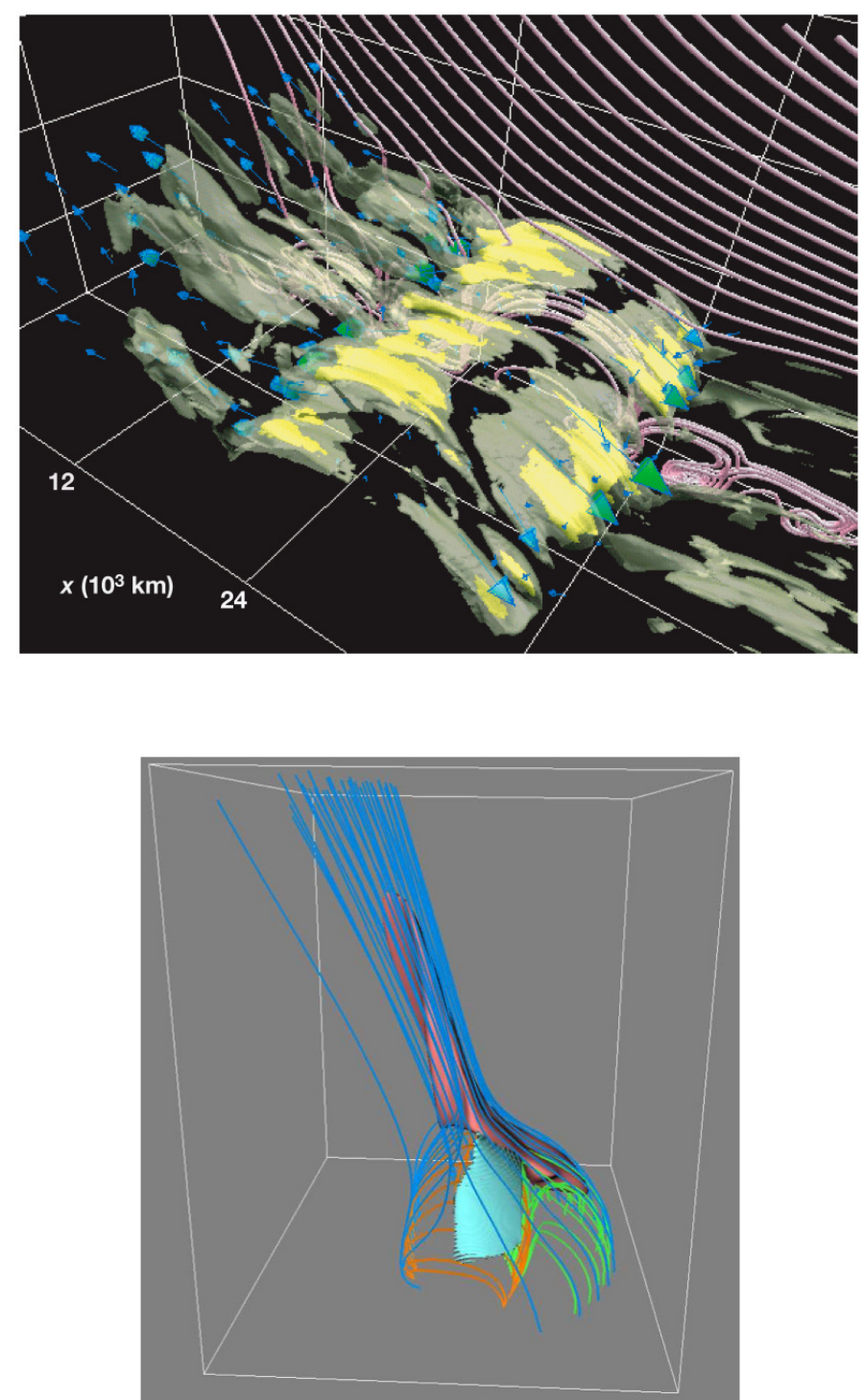

Figure 31: Interaction of emerging and preexisting magnetic fields in three-dimensional space. In the top panel, the color surfaces correspond to the isosurface of flow velocity, the arrows to the velocity field, and the white/pink lines to the field lines (from Isobe et al., 2005). In the bottom panel, the blue and pink surfaces represent $j / B$ and temperature, and the blue, orange, and green lines represent field lines in different connectivity domains (from Moreno-Insertis et al., 2008). 

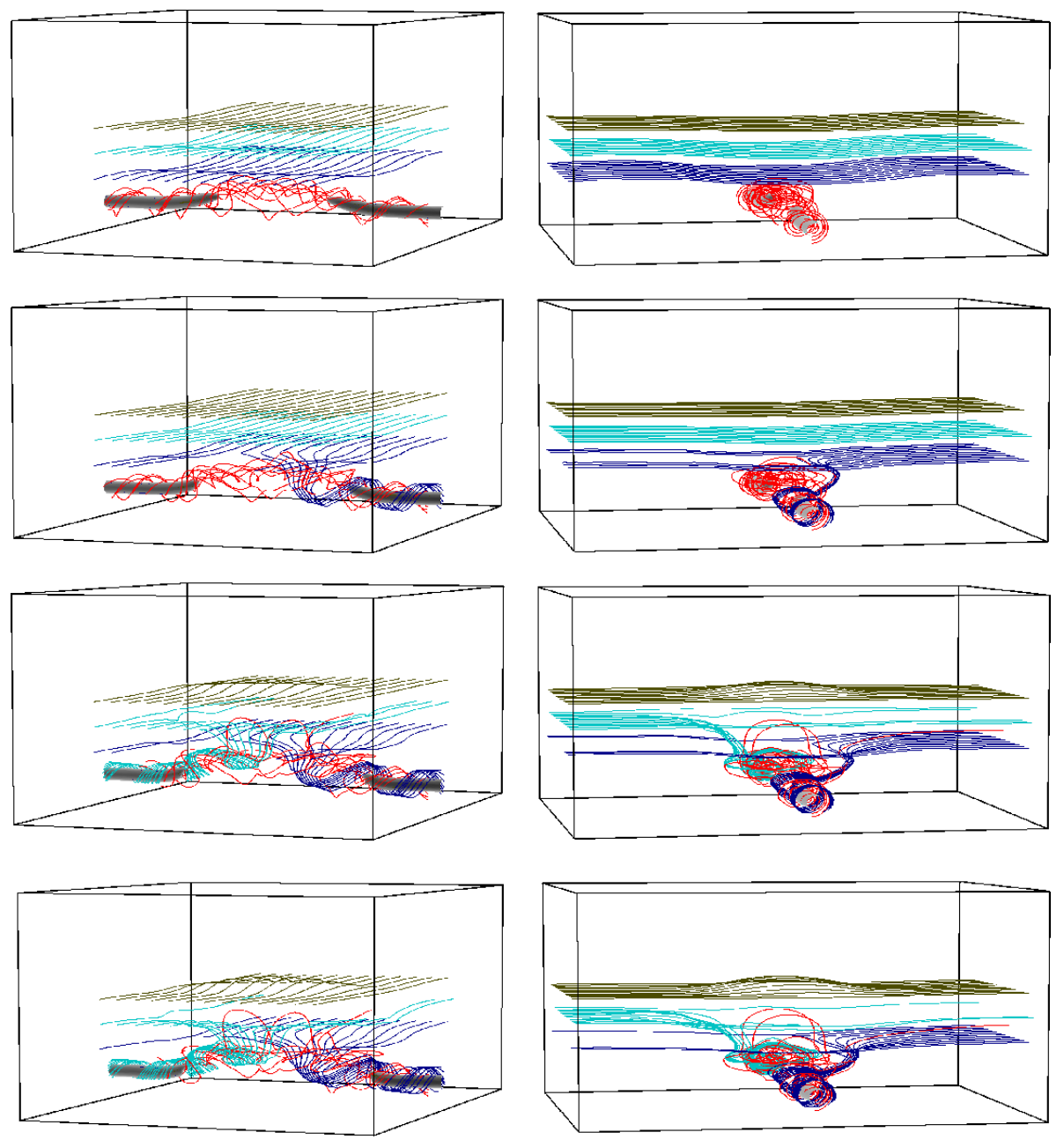

Figure 32: Interaction of emerging (red field lines) and preexisting magnetic fields (others) in threedimensional space (from Archontis et al., 2004). 


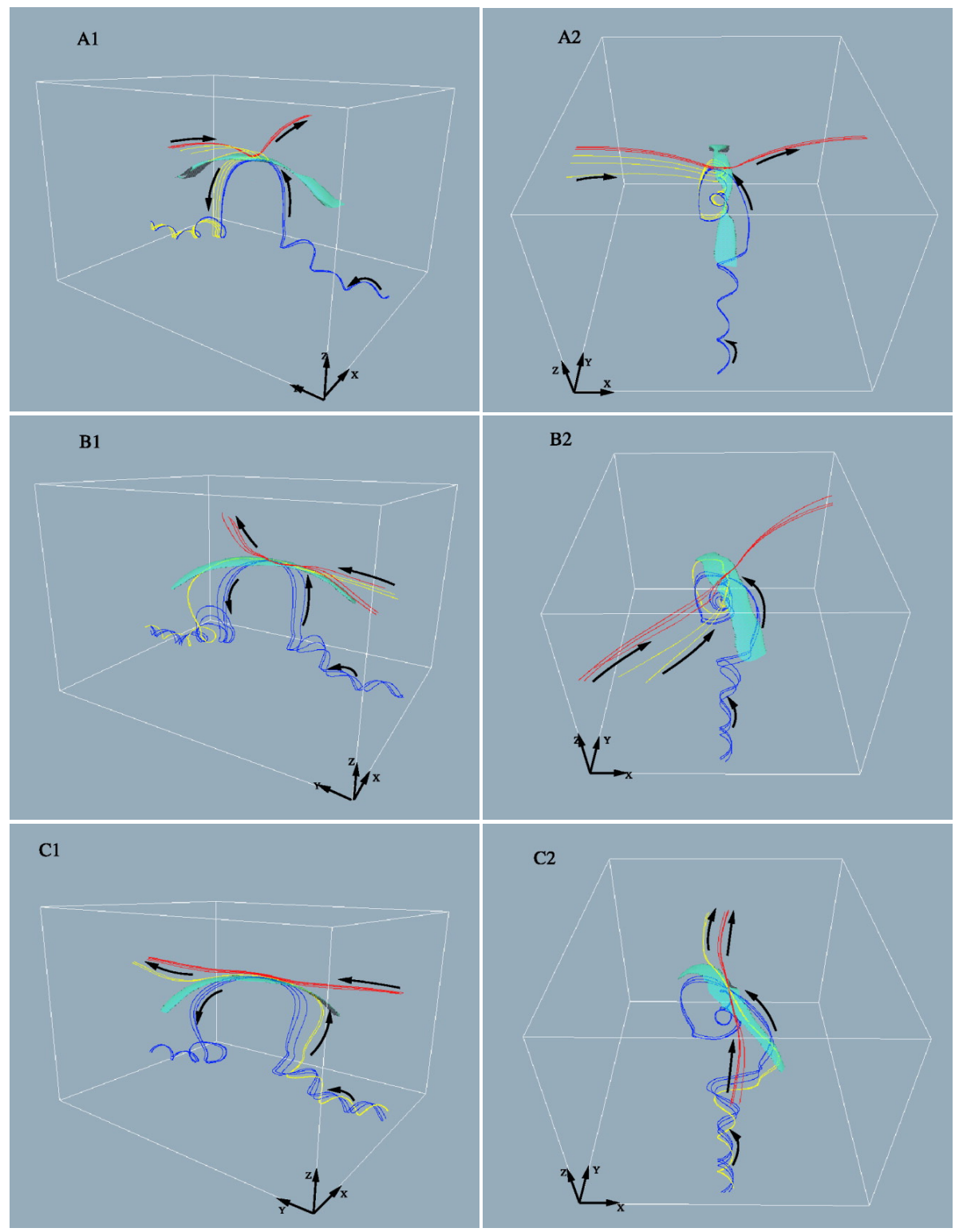

Figure 33: Interaction of emerging and preexisting magnetic fields in three-dimensional space. The lightblue isosurface represents a current sheet. The arrows show the direction of the magnetic field vector (from Galsgaard et al., 2007). 
configurations (Archontis et al., 2004; Isobe et al., 2005, 2006; Galsgaard et al., 2007; MorenoInsertis et al., 2008). Figures $31-33$ present several prominent results obtained from these threedimensional simulations.

Those works mentioned above are basically focused on the local dynamics produced by interacting flux domains, while it is also important to investigate the global magnetic configuration of multiple flux domains observed in real active regions. In particular, such a global approach permits to understand where current sheets are able to form, so where they locate flare ribbons/loops and how they evolve. It also permits a detailed comparison between modeling and observations (Aulanier et al., 2006; Masson et al., 2009, and references therein).

The so-called Minimum Current Corona (MCC) estimates the free energy stored in those multiple flux domains (see Longcope, 2005, for details). The original MCC assumes a configuration in which the magnetic field is in a potential state inside each flux domain, while it allows nonzero electric current flowing at the interface between flux domains. This gives a minimum excess of magnetic energy over the potential field energy in multiple flux domains. The MCC tells where current sheets form and how much electric current flows, so it can be used to investigate where flares might occur and how much energy will be released (Longcope, 1998). Later MCC was extended to enable the magnetic field to deviate from a potential state inside each flux domain (Longcope and Magara, 2004). While the MCC assumes a current sheet of infinitesimal thickness, there is also a model using a current sheet of finite thickness, which is called the quasi-separatrix layer (QSL) (Priest and Démoulin, 1995; Démoulin et al., 1996). Longcope (2005) is one of the comprehensive reviews on magnetic topology on the Sun.

\subsubsection{Development of magnetic shear in single flux domain}

The formation of a current sheet in a flux domain could be a direct consequence of the emergence of a twisted flux tube, as shown in Figure 22. A key process here is the development of magnetic shear inside a magnetic structure. This process has extensively been studied using the so-called shear-induced model in which shear and/or converging flows are imposed on the footpoints of coronal loops to increase magnetic shear (Mikić et al., 1988; Biskamp and Welter, 1989; DeVore and Antiochos, 2000; Inhester et al., 1992; Aly, 1995; Amari et al., 1996, 2003; Choe and Lee, 1996). An important suggestion from these works is that a current sheet tends to form as magnetic shear develops, followed by magnetic reconnection in the current sheet, leading to the onset of a flare (see Figure 34).

Later, the origin of these shear and converging flows assumed in the shear-induced model as photospheric boundary conditions has been investigated from the viewpoint of flux emergence. It has been demonstrated that the shear flows are self-consistently driven via the emergence of sheared magnetic structure, such as a twisted flux tube (Manchester IV, 2001; Fan, 2001; Magara and Longcope, 2001; Manchester IV et al., 2004; Manchester IV, 2007) (see Figure 35).

Regarding the converging flows, Magara (2011) demonstrates that the emergence of U-loops composing a twisted flux tube causes the convergence of photospheric footpoints of these loops, but the plasma actually shows a diverging motion on those loops which is opposite to the apparent converging motion of magnetic polarities at the surface (see Figure 36).

Manchester IV et al. (2004) show that a twisted flux tube forms a flux rope above the surface, which tends to keep floating in the corona (the rising velocity of the flux rope decreases toward zero with time). This suggests that a mechanism to eject a flux rope from the corona to the interplanetary space is needed. 
a)
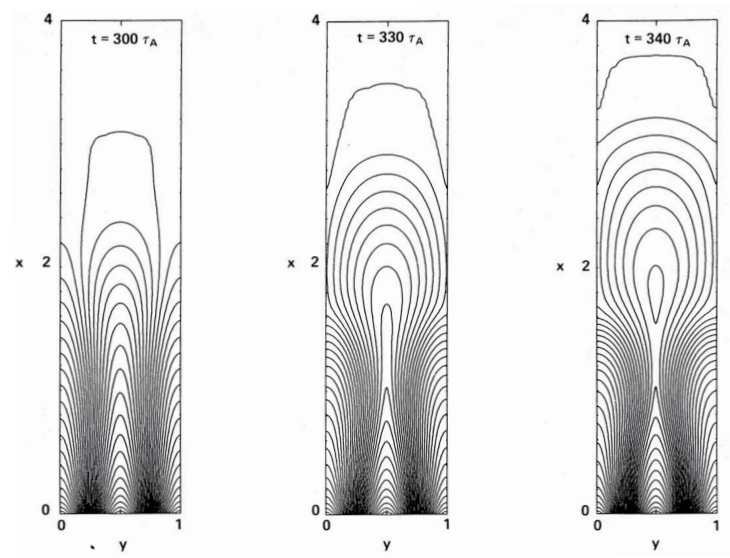

b)

c)
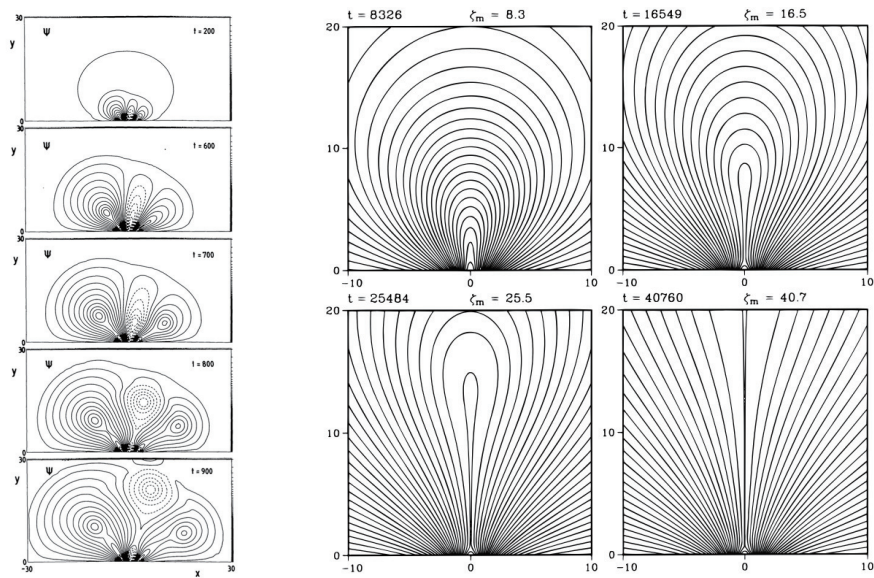

Figure 34: Shear-induced model for the formation of a current sheet. (a) From Mikić et al. (1988), (b) from Biskamp and Welter (1989), and (c) from Choe and Lee (1996) (bottom right).

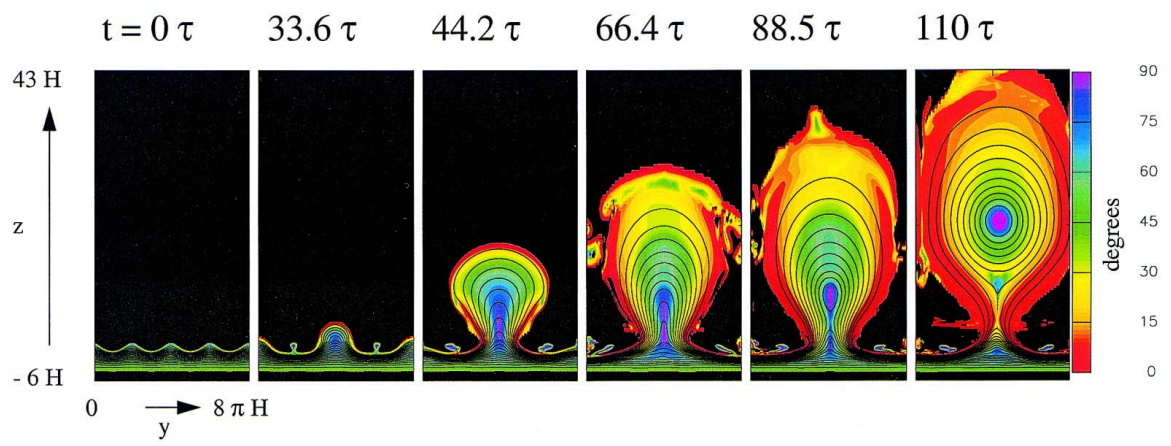

Figure 35: Formation of a magnetic flux rope by sheared magnetic field emerging into a stratified atmosphere. The colors indicate shear angle (from Manchester IV, 2001). 


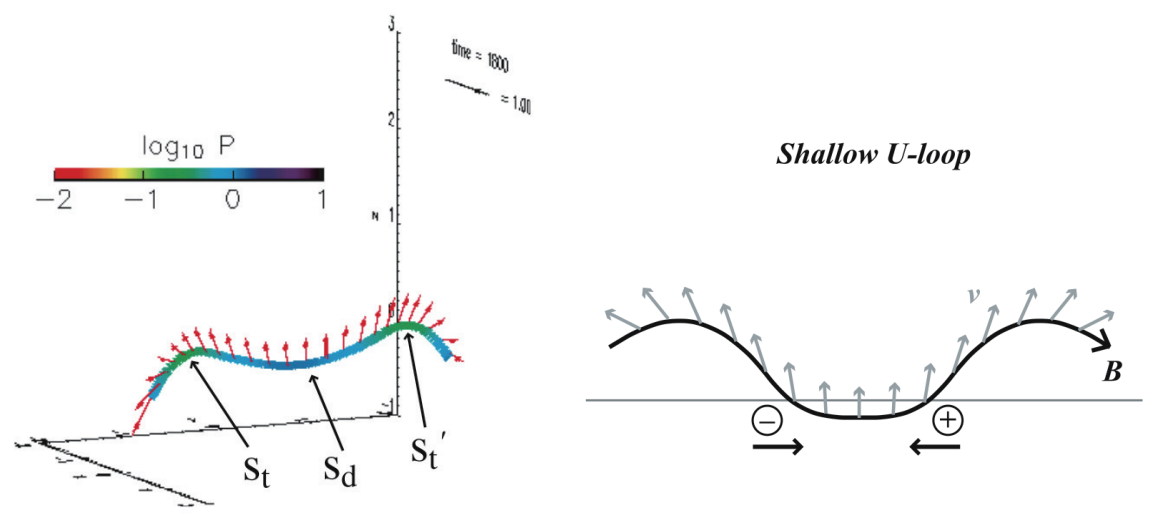

Figure 36: Emergence of an U-loop with diverging flow on it (from Magara, 2011).

\subsection{Modeling of energy-release processes}

The ejection of a flux rope to the interplanetary space has widely been investigated using various kinds of models. Coronal mass ejections (CMEs) are one of the direct manifestation of this ejection. Since the ejection occurs in the corona with low plasma beta, the main forces exerting on a flux rope are the gravitational force, gradient force of magnetic pressure, and magnetic tension force. Losing a balance among them causes the ejection of a flux rope.

\subsubsection{Bipolar system (single flux domain)}

Several models explain ejection in bipolar system. The so-called mass-loaded model assumes that the drain of plasma from a flux rope reduces the gravitational force, leading to the ejection of a flux rope via enhanced magnetic buoyancy (Low and Hundhausen, 1995; Low, 1996; Wu et al., 1997). The flux-cancellation model shows that the dissipation of magnetic flux at the surface (known as flux cancellation) reduces the magnetic tension force of the overlying field that confines a flux rope so that the upward magnetic pressure dominates at some point of the evolution and an eruption occurs (the configuration has a low beta plasma and magnetic force dominates) (Linker et al., 2003, see Figure 37a). A similar mechanism has been proposed in the so-called the tether-cutting model (Sturrock et al., 1984; Moore et al., 2001, see Figure 37b). If a flux rope is composed of highly twisted field lines, then the kink instability might develop, causing the ejection of it (Sturrock et al., 2001; Fan and Gibson, 2003; Török and Kliem, 2005; Inoue and Kusano, 2006, see Figure 37c). The basic physics related to the kink instability, or more precisely the toroidal magnetic force, has also been studied in early analytical models (Mouschovias and Poland, 1978; Chen, 1989; Vršnak, 1990, see also references therein).

The so-called loss-of-equilibrium model suggests that there is a critical height of a flux rope, beyond which no neighboring equilibrium state exists, so if a flux rope reaches this height, then a dynamic transition inevitably occurs to cause eruption (Forbes and Isenberg, 1991; Amari et al., 2000; Roussev et al., 2003; Lin, 2004; Isenberg and Forbes, 2007, see Figure 37d). In fact, the flux cancellation model mentioned above demonstrates the dynamic process of the loss of equilibrium.

Recently, it has been proposed that the torus instability plays a key role in the ejection of a flux rope (Kliem and Török, 2006). They suggest that the spatial distribution of the magnetic field overlying a flux rope is an important factor in controlling the dynamic state of a flux rope. The loss-of-equilibrium and the torus instability are in fact two different views of the same physical process (Démoulin and Aulanier, 2010). 
a)

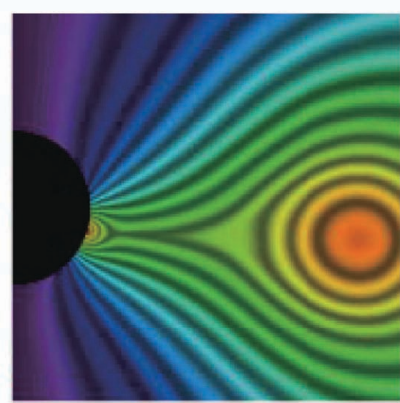

c)

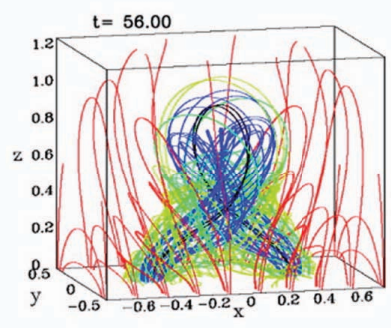

e)

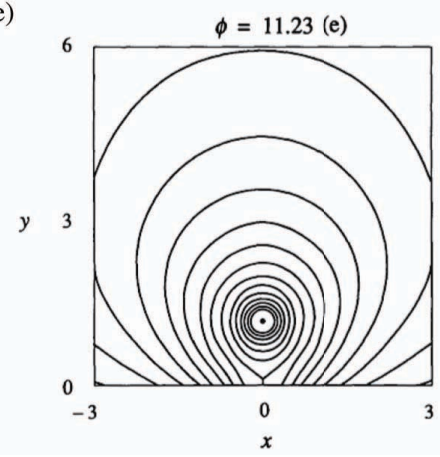

b)

\section{Eruption Onset}

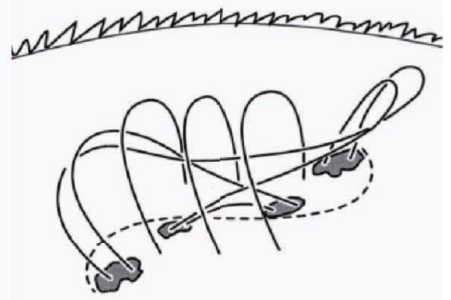

d)
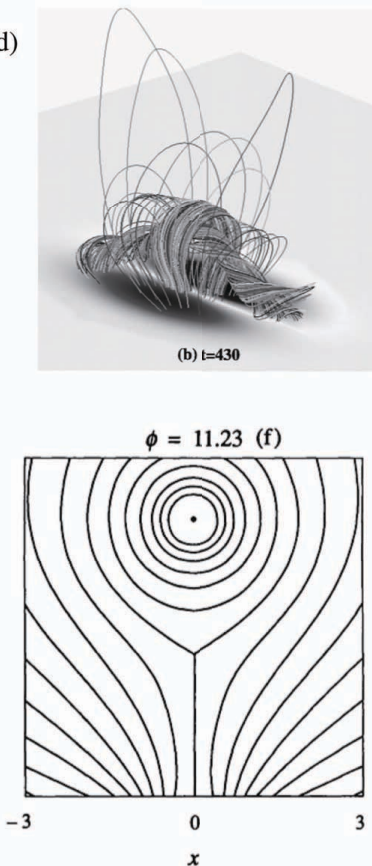

Figure 37: Models for the eruption of a flux rope. (a) Flux-cancellation model (from Linker et al., 2003). (b) Tether-cutting model (from Moore et al., 2001). (c) Kink instability (from Fan and Gibson, 2003). (d) Flux cancellation model (from Amari et al., 2000). (e) Loss-of-equilibrium model (from Forbes and Isenberg, 1991). 


\subsubsection{Multi-polar system (two-step reconnection)}

In multi-polar systems, the following models have been proposed to explain the ejection of a flux rope. In the model called breakout (Antiochos et al., 1999a, Figure 38), a current sheet is first formed at the interface between the inner and overlying flux domains. Magnetic reconnection then occurs at this current sheet, reducing the confining tension force of the overlying field. The inner domain then starts to expand, inside which another current sheet is formed and the second, much more energetic reconnection than the previous one, occurs to produce a flare. As a result of the second reconnection, a flux rope is formed, which eventually erupts into the interplanetary space.

Another model is called the emerging flux trigger model (Chen and Shibata, 2000, Figure 39), in which newly emerging magnetic field interacts with the preexisting field that contains a flux rope. That interaction leads to the formation of a current sheet at the interface between those two fields. Magnetic reconnection then occurs in this current sheet, destabilizing the flux rope, which erupts via the second reconnection that is similar to the one explained in the breakout model. In these two models, the two-step reconnection is a key mechanism for producing a flare and the ejection of a flux rope. This mechanism also works in the helicity annihilation model (Kusano et al., 2004, Figure 40).
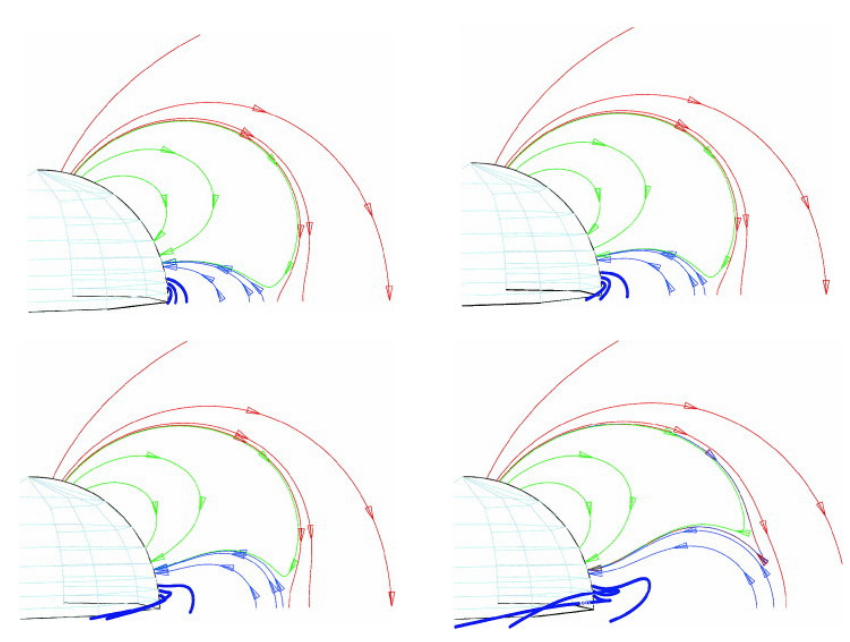

Figure 38: Destabilizing mechanism for the eruption of a flux rope, known as the breakout model presented in Antiochos et al. (1999a). 

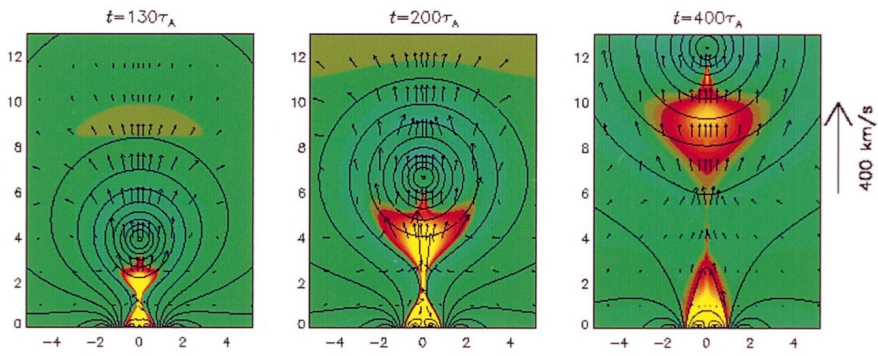

$t=250 \tau_{\mathrm{A}}$

$t=290 \tau_{\mathrm{R}}$
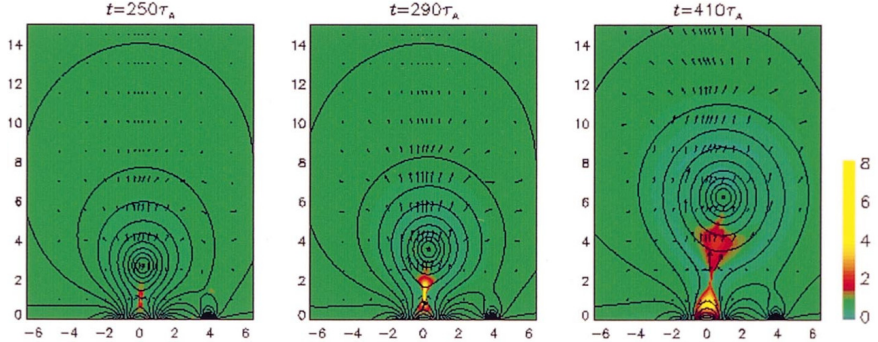

Figure 39: Destabilizing mechanism for the eruption of a flux rope, known as the emerging flux trigger mechanism presented in Chen and Shibata (2000). The solid lines correspond to the magnetic field, the arrows to the velocity, and the color map to the temperature. At the top panels, the emerging field appears just on the polarity inversion line of the preexisting field, while it appears at one side of the inversion line at the bottom panels.
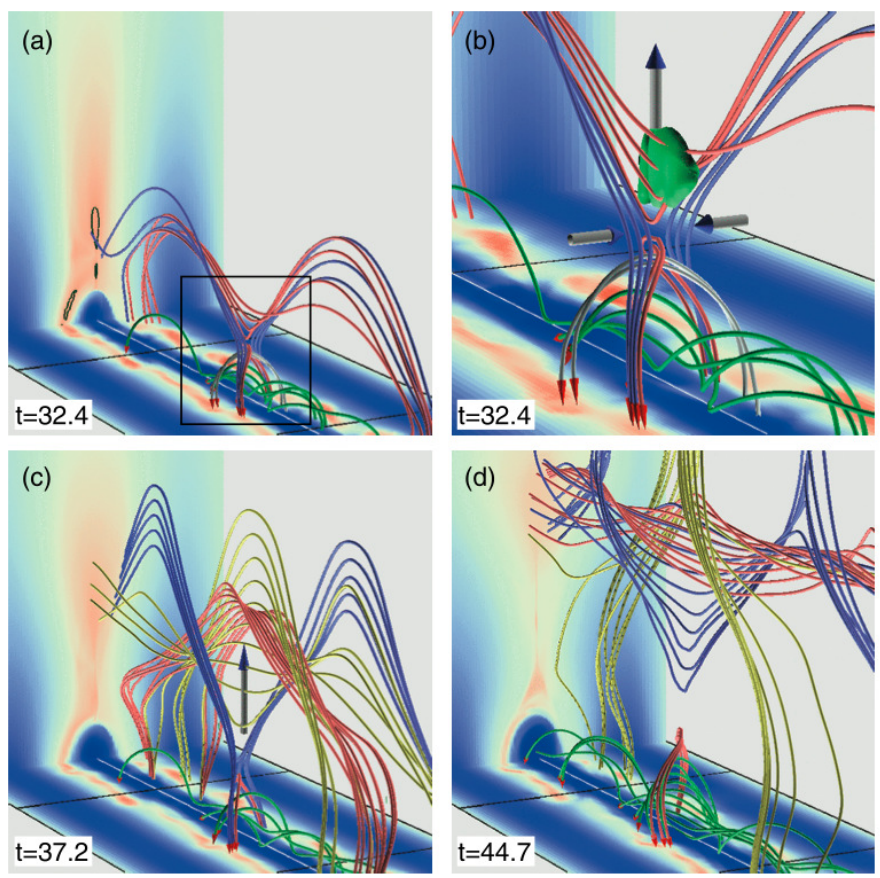

Figure 40: Destabilizing mechanism for the eruption of a flux rope, known as the reversed magnetic-shear model developed in Kusano et al. (2004). Typical plasma flows are illustrated by thick arrows. The green surface in (b) represents an isosurface on which $V_{z}=0.1 V_{A}$. The color maps on the side and bottom boundaries represent the flux density of sheared and vertical component of magnetic field. 


\section{$5 \quad$ Energy Transport}

The energy released by magnetic reconnection is transported in various ways, such as radiation, thermal conduction, mass ejection, wave propagation, and generation of high energy particles. The energy transporting process affects the solar atmosphere significantly, making the main phase of a flare rich with dynamic events such as shock formation, jet and plasmoid ejection, chromospheric evaporation, and acceleration of charged particles. In this section we explain these dynamic processes observed in a postflare phase.

\subsection{Radiation}

Figure 41 illustrates the time variation of emissions observed in various wavelengths during a flare. At the beginning of a flare, the rapid increase of hard X-ray (HXR) $(>30 \mathrm{keV})$ and microwave emissions is observed, which is called burst or elementary bursts (e.g., de Jager and Sakai, 1991). This event occurs recurrently during the period of several seconds. While a burst is occurring, high-energy particles are usually generated. The total duration of a series of bursts is about a few minutes, which forms the impulsive phase of a flare. During this phase the most violent energy release occurs. After that, emissions from a flaring site gradually decrease in about ten minutes, which is called the gradual phase of a flare. In some events, the total duration of a series of bursts might be longer than $10 \mathrm{~min}$. HXR and microwave emissions are strong only during the impulsive phase, while soft X-ray (SXR) $(<10 \mathrm{keV})$ and $\mathrm{H} \alpha$ emissions continue to increase after the impulsive phase and become dominant during the gradual phase.

Space observations such as SMM (1980-1989), Hinotori (1981-1989), and Yohkoh (19912001) have provided the detailed information on the structure and evolution of a flare, which enabled us to identify the source region of emissions observed during impulsive and/or gradual phases. Figure 42 illustrates the configuration of magnetic field observed during these two phases, in addition to the source region of emissions observed in various wavelengths.

An HXR source is formed at the chromospheric footpoint of a loop observed in soft X-rays (SXR loop), which is called HXR footpoint source. High-energy electrons generated by magnetic reconnection in the corona are supposed to stream downward along an SXR loop, heating chromospheric plasma to form an HXR footpoint source there. These high-energy electrons also produce microwave emissions intermittently via gyro-synchrotron radiation while streaming downward along an SXR loop. Another type of HXR sources is formed above the top of an SXR loop, which is called HXR loop-top source (Masuda et al., 1994). The HXR loop-top source may be formed by a downward high-speed flow (jet) which has been produced by magnetic reconnection and collides with the top of an SXR loop. The collapsing trap effect may be occurring during this phase near the top of the SXR loop (Somov and Kosugi, 1997; Karlický and Kosugi, 2004; Veronig et al., 2006). Soft X-ray emissions start to increase gradually during the preflare phase of a flare, suggesting that plasma heating already occurs before the onset of a flare. During the impulsive phase, the intensity of soft X-ray emissions increases rapidly and the time derivative of the soft $\mathrm{X}$-ray intensity rise corresponds to the time variation of hard X-ray emissions, which is known as Neupert effect (Neupert, 1968). The main contribution to producing these soft X-ray emissions comes from a loop filled with hot plasma whose temperature is about $T \geq 10^{7} \mathrm{~K}$. This plasma originally comes from the chromosphere via evaporation driven by the thermal conduction (and high-energy electrons in part) emanating from a super-hot region formed in the corona (around the region where magnetic reconnection occurs). The thermal conduction also continuously heats the evaporated plasma and tries to keep its coronal temperature (eventually the evaporated plasma reduces its temperature by radiative cooling and forms $\mathrm{H} \alpha$ loops).

During the impulsive phase, bright kernels are observed in $\mathrm{H} \alpha$ at the footpoints of an SXR loop. This also indicates the heating of chromospheric plasma by thermal conduction and high- 


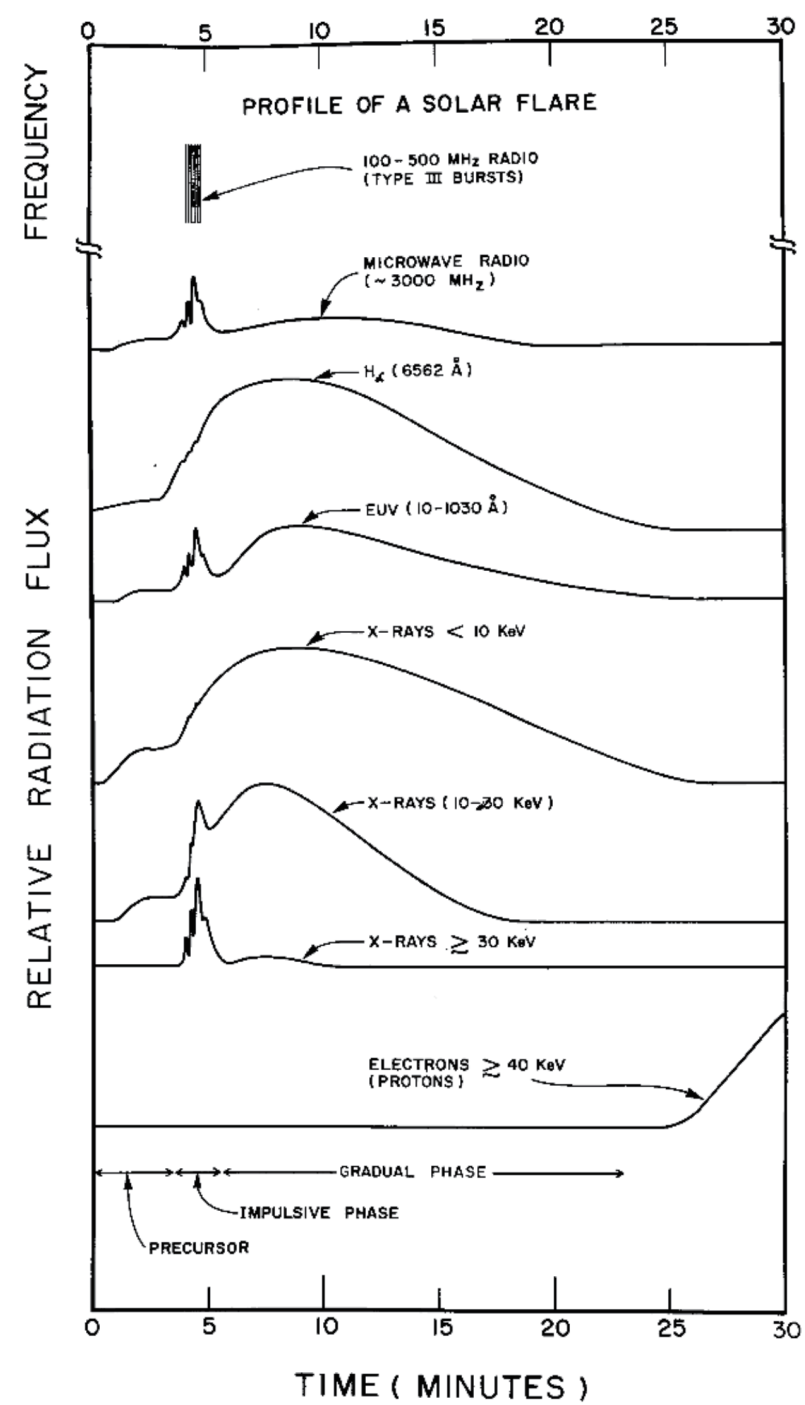

Figure 41: Typical time variation of emissions observed in various wavelengths during a flare (from Kane, 1974). 


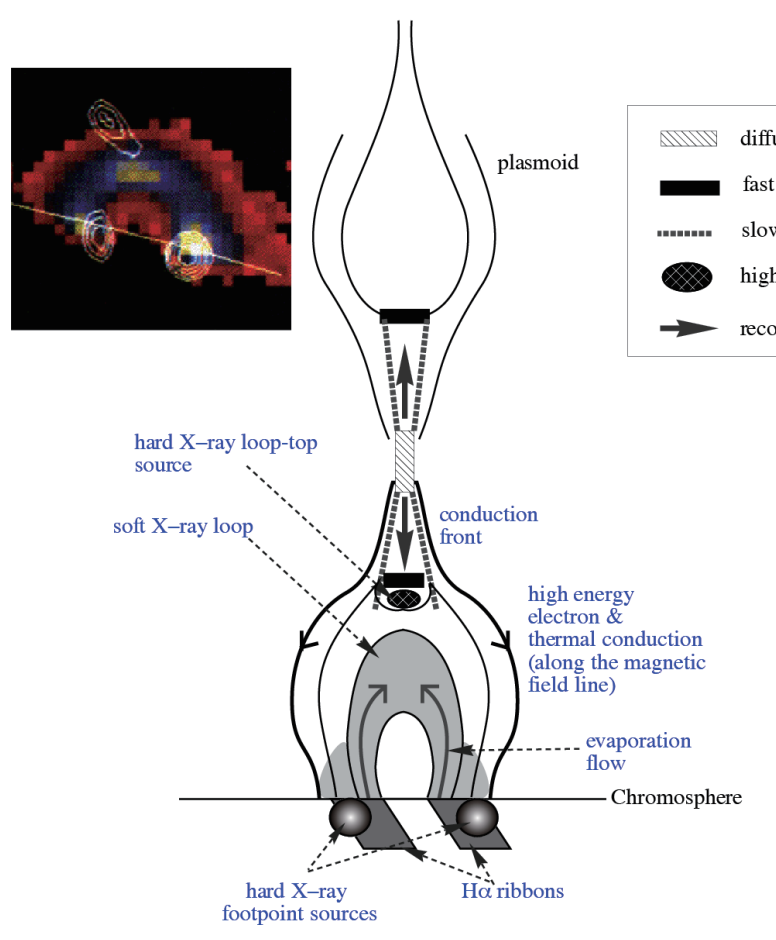

Impulsive phase (or impulsive flare)
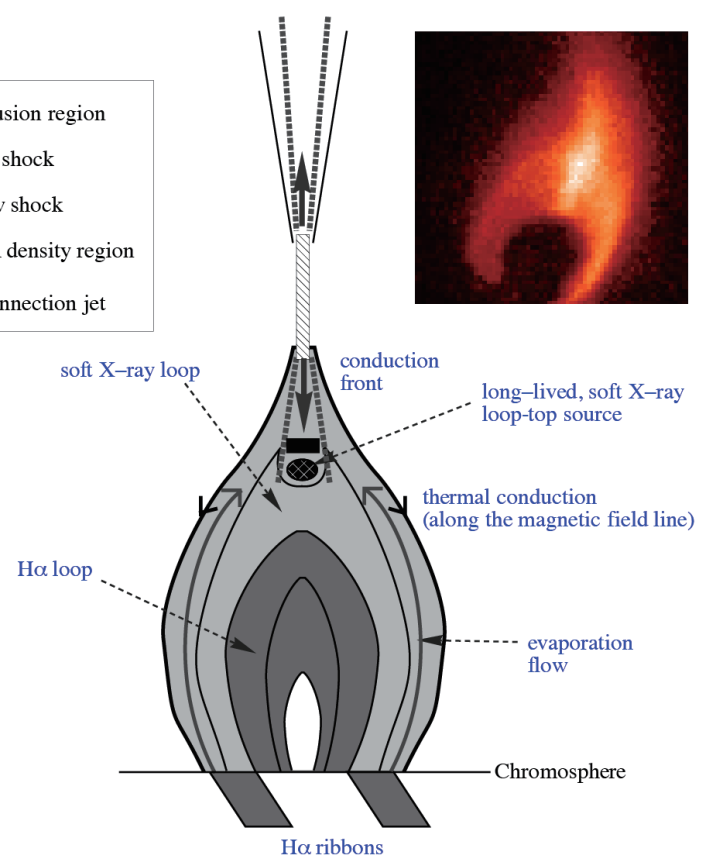

Gradual phase (or LDE flare)

Figure 42: Magnetic configurations in the impulsive (left panel) and gradual (right panel) phases of a flare. The source region of emissions observed in various wavelengths are also presented. These two kinds of configurations are applied to different types of flares such as impulsive flares and LDE flares (adapted from Magara et al., 1996). An observational example of these flares are displayed at the top left (Masuda, 1994) and top right (Tsuneta et al., 1992a), respectively.

energy electrons. It has often been argued that explosive evaporation in the impulsive phase is primarily due to electron beams, causing Neupert effect, whereas the gentle evaporation in the gradual phase is due to conduction (e.g., Veronig et al., 2010, and references therein). When a group of SXR loops appear almost simultaneously and form an arcade, the $\mathrm{H} \alpha$ kernels are observed as two ribbons distributed along the polarity inversion line (called H $\alpha$ ribbons, see Figure 1 ). In an extremely energetic case, high-energy electrons can penetrate the chromosphere and heat the photosphere, causing the enhancement of white-light emissions (white-light flare).

$\mathrm{H} \alpha$ emissions are also observed during the gradual phase of a flare. In this case the main contribution comes from a loop filled with cool plasma with $T \sim 10^{4} \mathrm{~K}$ (H $\alpha$ loop). An $\mathrm{H} \alpha$ loop starts to appear when an SXR loop experiences sufficient cooling via radiation and now is observed in $\mathrm{H} \alpha$. As magnetic reconnection proceeds in the corona, a newly reconnected field line successively piles up on a preexisting SXR loop, so the apparent height of an observed SXR loop increases with time. In accordance with this, the distance between the two $\mathrm{H} \alpha$ ribbons observed at both footpoints of an $\mathrm{H} \alpha$ loop also increases with time (see Figure 1). The postflare loops are seen on the disc in emission in $\mathrm{H} \alpha$ only if they are dense enough (say $n>10^{12} \mathrm{~cm}^{-3}$; Švestka, 1976) which happens only in very powerful flares, so this is a rather rare phenomenon. 


\subsection{Mass ejection}

\subsubsection{Reconnection jet}

Magnetic reconnection produces oppositely directed, bidirectional high-speed flows (called reconnection jets) emanating from the reconnection point. The velocity of reconnection jet is given by the Alfvén velocity in Equation (22). In the case of the Sweet-Parker-type reconnection, the width of reconnection jet is nearly constant, comparable to the width of the diffusion region (see Figure 26)

$$
w_{\text {jet }} \simeq M_{A} L \sim 100 \mathrm{~cm}
$$

where $M_{A}$ is the nondimensional reconnection rate given in Equation (19) and $L$ (length of the diffusion region, see Figure 26) $\sim 10^{9} \mathrm{~cm}$. On the other hand, the Petschek-type reconnection gives

$$
w_{\text {jet }} \simeq M_{A} L \sim 100-1000 \mathrm{~km},
$$

where $M_{A}$ is the nondimensional reconnection rate given in Equation (21). Since the Petschektype reconnection is accompanied by the slow MHD shocks that extend from the diffusion region (explained below) and contribute to accelerating plasma, the Petschek-type reconnection is more dynamic than the Sweet-Parker-type reconnection. It should be noted that the width of the reconnection jets produced by the Petschek-type reconnection is not constant, rather it increases as the jet leaves away from the reconnection point.

\subsubsection{Plasmoid ejection}

Magnetic reconnection mainly converts magnetic energy into thermal and kinetic energy, and part of the kinetic energy is used for plasmoid ejection. An observational result on plasmoid ejection and its comparison to theoretical modeling are presented in Figure 43. The dynamics of an ejecting plasmoid has been investigated in numerical simulations (Magara et al., 1997; Choe and Cheng, 2000; Shibata and Tanuma, 2001).

Figure 43a shows a result from a two-dimensional MHD simulation, in which magnetic reconnection produces an ejecting magnetic island (two-dimensional counterpart of a plasmoid). The time variation of the convective electric field defined by Equation (16) is also plotted at this panel. Figure 43b shows the height-time relations of an observed plasmoid as well as hard X-ray intensity (Ohyama and Shibata, 1997). When comparing these simulation and observation, we assume that the time variation of the convective electric field is closely related to the time variation of hard $\mathrm{X}$-ray emissions because the electric field can accelerate particles which contribute to producing hard X-ray emissions. The comparison suggests that the plasmoid ejection drives fast magnetic reconnection. More detailed investigations of plasmoid ejection are given by Choe and Cheng (2000), where multiple ejection of plasmoids and associated HXR bursts are discussed (see Figure 43c).

Shibata and Tanuma (2001) gives a rough estimate on the velocity of an ejecting plasmoid as follows:

$$
v=\frac{v_{A} \exp (\omega t)}{\exp (\omega t)-1+v_{A} / v_{0}}
$$

where $v_{0}$ and $v_{A}$ represent the initial velocity of a plasmoid and Alfvén velocity. In Equation (26), $\omega$ represents the velocity growth rate of a plasmoid, defined as

$$
\omega=\frac{\rho_{0} v_{A}}{\rho_{p} L}
$$

where $\rho_{0}$ is the density of ambient plasma while $\rho_{p}$ and $L$ are the density and length of a plasmoid.

Living Reviews in Solar Physics

http://www. livingreviews .org/lrsp-2011-6 
a)

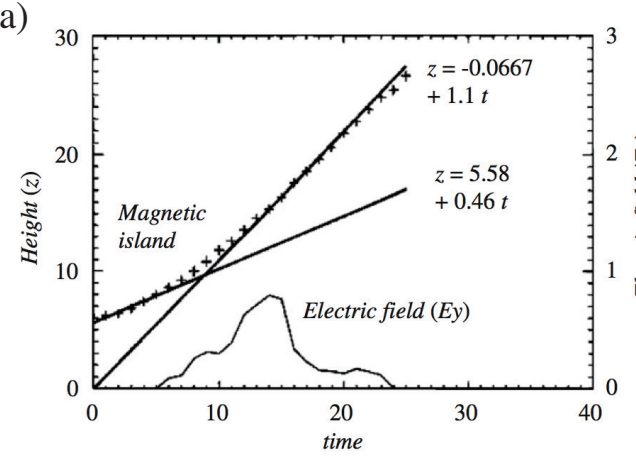

b)

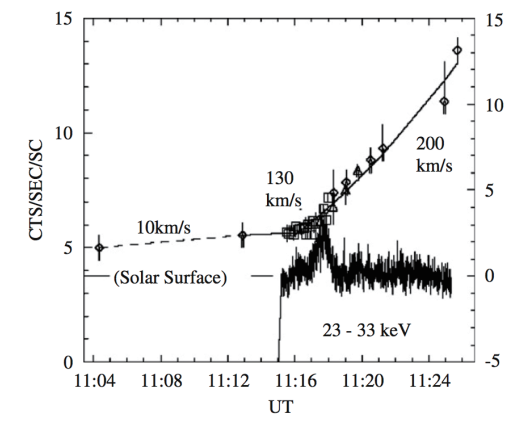

c)
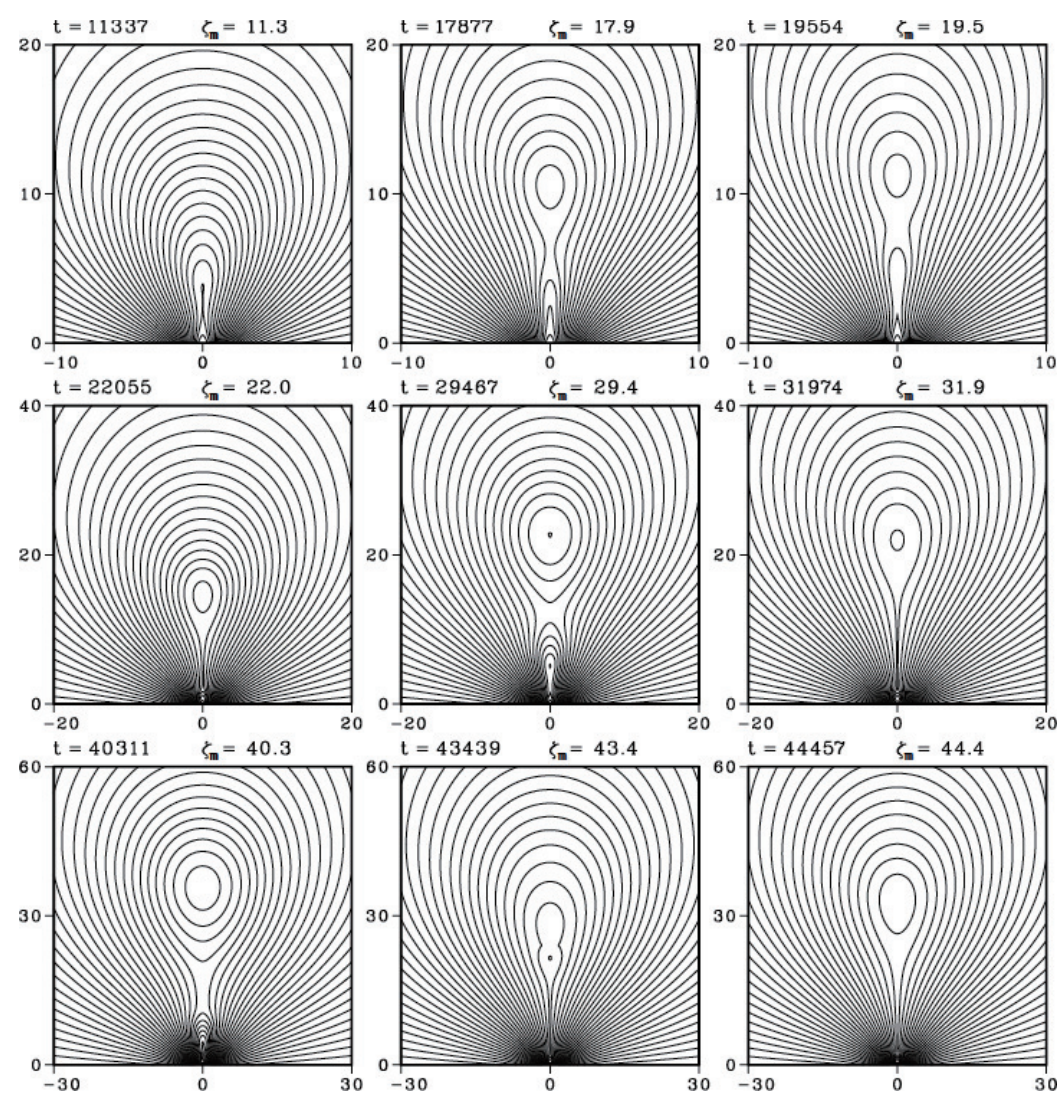

Figure 43: (a) Hight-time relation of a magnetic island in a two-dimensional numerical simulation, which is supposed to be the two-dimensional counterpart of a plasmoid. Time variation of the conductive electric field defined by Equation (16) is also plotted (from Magara et al., 1997). (b) Time variations of the height of an observed plasmoid as well as hard X-ray intensity (modified from Ohyama and Shibata, 1997). (c) Multiple ejection of plasmoids (from Choe and Cheng, 2000). 


\subsection{Shock formation and heating}

\subsubsection{Slow shock}

One of the important features of the Petschek-type reconnection is the formation of a pair of slow MHD shocks extending from a diffusion region. The role of the slow shocks was first considered in Cargill and Priest (1982). A pair of the slow shocks guide a reconnection jet, and the angle between these shocks is very narrow, of the order of $M_{A}$. In the adiabatic case, the temperature of coronal plasma increases across a slow shock up to (Vršnak and Skender, 2005)

$$
T_{\text {slow shock }} \sim T_{\text {corona }} / \beta,
$$

where

$$
\beta \equiv p_{\text {gas }} / p_{\text {mag }}=\frac{2 n k T}{B^{2} / 8 \pi} \sim 0.01\left(\frac{n}{10^{10} \mathrm{~cm}^{-3}}\right)\left(\frac{T}{10^{6} \mathrm{~K}}\right)\left(\frac{B}{100 \mathrm{G}}\right) .
$$

Here, we assume that the total pressure is balanced between the pre-shock region $\left(\sim B^{2} /(8 \pi)\right.$ because of low $\beta$ plasma) and post-shock region $\left(\sim p_{\text {gas }}\right.$ because of high $\left.\beta\right)$, and the gas density is roughly constant across the shock within a factor of 2-3. Equations (28) and (29) suggest that coronal plasma with $T \sim 1 \mathrm{MK}$ could be heated up to $100 \mathrm{MK}$. However, in reality, the thermal conduction works to reduce the temperature, so the value mentioned above is somewhat overestimated, just presenting the upper limit of the temperature enhanced via slow shock heating. Moreover, when the thermal conduction is efficient, an adiabatic slow shock tends to be split into a conduction front and an isothermal slow shock across which only the density and pressure increase but the temperature does not change (Forbes et al., 1989; Yokoyama and Shibata, 1997).

Yokoyama and Shibata (1997) first carried out a self-consistent MHD simulation of magnetic reconnection that includes thermal conduction (see the top panels in Figure 44). They confirmed that an adiabatic slow shock is split into a conduction front and an isothermal slow shock, as predicted by Forbes and Malherbe (1986). Yokoyama and Shibata (1997) further explained the structure of a cusp-shaped flare observed by Yohkoh (Tsuneta et al., 1992a), where the evaporation of chromospheric plasma heated by the conduction front is reproduced (Yokoyama and Shibata, 1998, see the bottom panels in Figure 44). Typically, the conduction time is estimated to be

$$
t_{\text {cond }} \sim \frac{3 n k_{B} T}{\kappa_{0} T^{7 / 2} / L^{2}} \sim 10\left(\frac{n}{10^{10} \mathrm{~cm}^{-3}}\right)\left(\frac{T}{10^{7} \mathrm{~K}}\right)^{-5 / 2}\left(\frac{L}{10^{9} \mathrm{~cm}}\right)^{2} \mathrm{~s}
$$

where $\kappa_{0} \simeq 10^{-6}$ (CGS) is the heat conductivity coefficient due to the coulomb collision (Spitzer, 1962) and $k_{B}$ is the Boltzmann constant. Here, we note that thermal flux saturation could occur at the conduction front in the presence of strong temperature gradients (e.g., Manheimer and Klein, 1975, for application see, e.g., Somov and Titov, 1985; Vršnak, 1989).

On the other hand, the Alfvén transit time is

$$
t_{A} \sim L / v_{A} \sim 10\left(\frac{n}{10^{10} \mathrm{~cm}^{-3}}\right)^{1 / 2}\left(\frac{B}{50 \mathrm{G}}\right)^{-1}\left(\frac{L}{10^{9} \mathrm{~cm}}\right) \mathrm{s} .
$$

For $T>10^{7} \mathrm{~K}$, the conduction time is shorter than the Alfvén transit time, so the effect of thermal conduction should be taken into account in considering the evolution of a flare.

The radiative cooling time of plasma is written as

$$
t_{\mathrm{rad}} \simeq \frac{3 k T}{n Q(T)} \simeq 5 \times 10^{3} \mathrm{~s}\left(\frac{T}{10^{6} \mathrm{~K}}\right)^{3 / 2}\left(\frac{n}{10^{9} \mathrm{~cm}}\right)^{-1} .
$$

Here,

$$
Q(T) \sim 10^{-22}\left(\frac{T}{10^{6} \mathrm{~K}}\right)^{-1 / 2} \operatorname{cgs} \text { for } T<10^{7} \mathrm{~K}
$$

Living Reviews in Solar Physics

http://www . livingreviews . org/lrsp-2011-6 

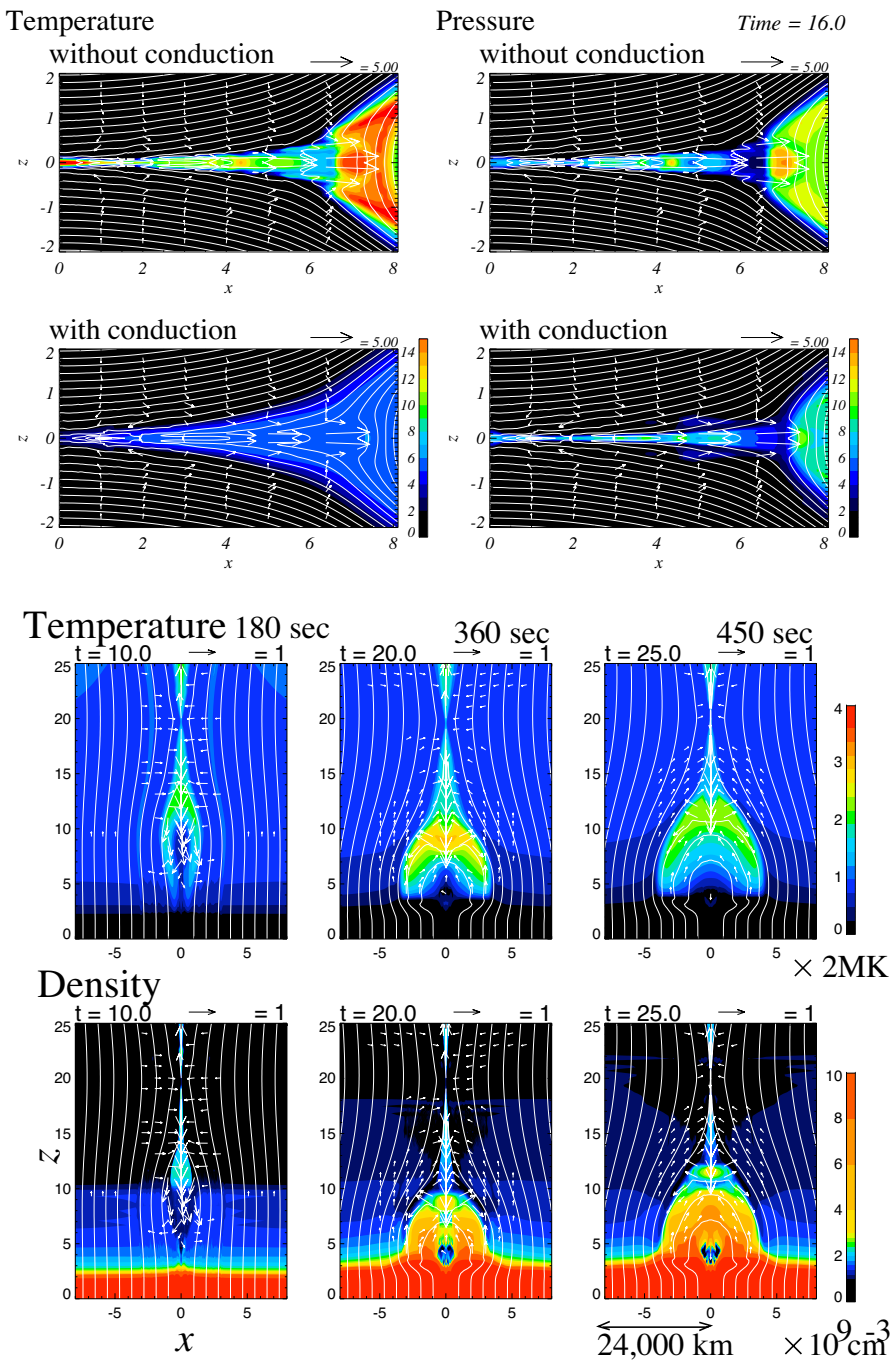

Figure 44: Top panels show temperature (left) and pressure (right) distributions in the Petschek-type reconnection for the cases without and with conduction. The curves show magnetic field lines and the arrows show velocity vectors. Note that the adiabatic slow shocks dissociate into the conduction fronts and the isothermal slow shocks in the case with conduction (from Yokoyama and Shibata, 1997). Bottom panel shows the time evolution of the temperature and density distributions in the reconnection with thermal conduction and chromospheric evaporation as a model of solar flares. The curves show magnetic field lines and the arrows show velocity vectors. In this case, not only the conduction fronts and isothermal slow shocks but also dense chromospheric evaporation flow are clearly seen (from Yokoyama and Shibata, 1998). 


$$
Q(T) \sim 3 \times 10^{-23}\left(\frac{T}{10^{7} \mathrm{~K}}\right)^{1 / 2} \operatorname{cgs} \text { for } T>10^{7} \mathrm{~K}
$$

is the radiative loss function for optically thin plasma (Raymond et al., 1976). In a typical SXR loop, the temperature is about $10^{7} \mathrm{~K}$ and electron density is about $n=10^{10} \mathrm{~cm}^{-3}$, so the radiative cooling time becomes of the order $10^{4} \mathrm{~s}$, which is much longer than both the conduction time and the Alfvén transit time. Hence, the radiative cooling could be neglected at least in the very early phase of a flare.

Assuming that cooling via thermal conduction $\left(\kappa_{0} T^{7 / 2} / L^{2}\right)$ is balanced by heating via magnetic reconnection $\left(Q\right.$ erg $\left.\mathrm{cm}^{-3} \mathrm{~s}^{-1}\right)$ in an SXR loop with the size of $L$, the temperature in this loop is given by (Fisher and Hawley, 1990)

$$
T_{\text {loop }} \sim\left(Q L^{2} / \kappa_{0}\right)^{2 / 7}
$$

The heating rate based on magnetic reconnection is given by

$$
Q \sim B^{2} v_{A} /(4 \pi L)
$$

This expression has been derived by Yokoyama and Shibata (1998) for the Petschek-type reconnection with a pair of slow shocks. In this case, the volumetric heating rate due to reconnection is given by the Poyinting flux entering into the reconnection region divided by the thickness of the reconnection region (bounded by a pair of the slow shock):

$$
Q=\left[B^{2} /(4 \pi)\right]\left(V_{\text {in }} / L\right)(1 / \sin \theta),
$$

where $V_{\text {in }}$ is the velocity of the inflow into the slow shock, $L$ is the length of the slow shock and $\theta$ is the angle between the slow shock and the initial field line. Since the $\theta$ is approximately given by $\sin \theta \approx V_{\text {in }} / V_{A}$, we have Equation (36). Note that this is a crude estimate, though it is roughly consistent with the numerical simulation results of Yokoyama and Shibata $(1998,2001)$ within a factor of a few.

Combining Equation (35) and (36), we obtain

$$
\begin{aligned}
T_{\text {loop }} & \sim\left(\frac{B^{2} v_{A} L}{4 \pi \kappa_{0}}\right)^{2 / 7} \\
& \sim 4 \times 10^{7}\left(\frac{B}{100 \mathrm{G}}\right)^{6 / 7}\left(\frac{n}{10^{10} \mathrm{~cm}^{-3}}\right)^{-1 / 7}\left(\frac{L}{10^{9} \mathrm{~cm}}\right)^{2 / 7} \mathrm{~K} .
\end{aligned}
$$

These values are measured behind the slow shock from which the conduction front and isothermal slow shock extend (Forbes et al., 1989; Yokoyama and Shibata, 1998, 2001). The temperature of $10^{7} \mathrm{~K}$ is comparable to the one observed in the superhot region formed at the top of an SXR loop (Masuda, 1994; Kosugi et al., 1994; Tsuneta, 1996; Nitta and Yaji, 1997). Note that this value is smaller than the value obtained from adiabatic MHD simulations, in which the temperature becomes of the order $10^{8} \mathrm{~K}$ as mentioned before.

It should be noted that the conduction could be strongly reduced due to a large difference in the magnetic field strength in inflow and outflow region, as well as due to thermal flux saturation and the flow/field geometry (for details see Vršnak et al., 2006, and references therein).

\subsubsection{Chromospheric evaporation}

During a flare, the chromospheric evaporation (i.e., ablation of chromospheric plasma) plays a fundamental role in creating an SXR loop via the injection of hot plasma into a loop (Hirayama, 1974). The evaporation occurs when the heat flux coming from the corona overcomes radiative cooling rate in the chromosphere. That heat flux then increases the gas pressure in the upper chromosphere significantly to produce an upflow toward the corona against gravity (called evaporation

Living Reviews in Solar Physics

http://www. livingreviews .org/lrsp-2011-6 
flow Antonucci et al., 1982, 1984) as well as downflow (Ichimoto and Kurokawa, 1984; Canfield et al., 1990) to the lower chromosphere. In a steady state, the heat flux of thermal conduction from the corona is balanced by the enthalpy flux of evaporation, such as

$$
\kappa_{0} T^{7 / 2} / L^{2} \sim \frac{5}{2} p v_{\text {evap }}
$$

Here $v_{\text {evap }}$ is the velocity of an evaporation flow, which is the same order of the sound velocity,

$$
v_{\text {evap }} \sim c_{s} \sim 500\left(\frac{T}{10^{7} \mathrm{~K}}\right)^{1 / 2} \mathrm{~km} \mathrm{~s}^{-1},
$$

since the flow is driven by gas pressure. When the heat flux reaches a deep layer in the chromosphere and is balanced by enhanced radiative cooling rate due to large gas density, evaporation ceases.

Nagai (1980) first performed a one-dimensional hydrodynamic simulation of chromospheric evaporation. Since then, similar one-dimensional hydrodynamic simulations have been performed extensively (Somov et al., 1981; Nagai and Emslie, 1984; Peres et al., 1987; MacNeice et al., 1984; Mariska et al., 1989; Fisher and Hawley, 1990; Gan et al., 1991), which qualitatively explained the blue shift of Bragg Crystal Spectrometer (BCS) lines observed by Yohkoh as well as the red shift of $\mathrm{H} \alpha$ line observed during the impulsive phase of a flare (Ichimoto and Kurokawa, 1984). Investigations into the quantitative agreement between one-dimensional models and observations are still in progress. Later, pseudo two-dimensional models have been developed, reported by several authors (Hori et al., 1997; Warren et al., 2003). Yokoyama and Shibata (1998) performed a two-dimensional MHD simulation reproducing the chromospheric evaporation driven by thermal conduction (see the bottom panels in Figure 44). By combining magnetic reconnection, thermal conduction and radiative cooling, they derived a scaling law about the temperature observed in a loop filled with evaporated plasma, as shown in Equation (38). Later, Shibata and Yokoyama (1999) applied this scaling law to stellar flares (see Section 6).

\subsubsection{Fast shock}

A fast MHD shock is formed by the downward reconnection jet colliding with the top of an SXR loop (Forbes and Priest, 1983; Ugai, 1987; Magara et al., 1996; Aurass et al., 2002) where highenergy electrons are possibly produced (see Figure 42). In the adiabatic case, the temperature just behind the fast shock becomes

$$
T_{\text {fast shock }} \sim m_{i} v_{\text {jet }}^{2} /\left(6 k_{B}\right) \sim 2 \times 10^{8}\left(\frac{B}{100 \mathrm{G}}\right)^{2}\left(\frac{n_{e}}{10^{10} \mathrm{~cm}^{-3}}\right)^{-1} \mathrm{~K},
$$

where $m_{i}$ is the proton mass (Vršnak and Skender, 2005). This value might be overestimated because the thermal conduction is not taken into account, unless magnetic field lines are so tangled as to reduce the efficiency of thermal conduction.

A fast shock could also be formed at the bottom of an ejecting plasmoid when it moves much slowly compared to the local Alfvén velocity (Magara et al., 1997, 2000).

\subsection{Wave propagation}

Wave propagation is another type of dynamic phenomena observed after the onset of a flare. The so-called Moreton wave is one of these waves, which is observed in H $\alpha$ (Moreton and Ramsey, 1960; Moreton, 1960). Moreton waves are often associated with type II radio bursts (e.g., Uchida, 1974). The mechanism for producing the Moreton wave is given by Uchida (1968), where this wave is explained as the chromospheric counterpart of a fast MHD shock wave propagating in the corona. Recent observations of the Moreton wave based on high temporal resolution have revealed various 
aspects of this highly transient phenomenon (Khan and Hudson, 2000; Warmuth et al., 2001; Eto et al., 2002; Narukage et al., 2002; Okamoto et al., 2004; Warmuth, 2007). Figure 45 shows an observational result on the structure and evolution of a Moreton wave, derived from a detailed analysis of $\mathrm{H} \alpha$ and soft X-ray data.

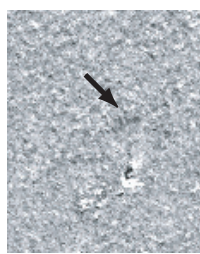

(a) 04:36:01
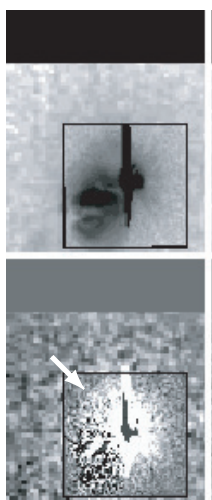

(g) 04:35:26
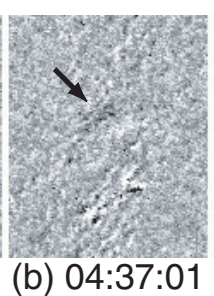

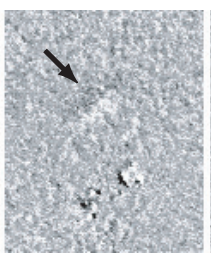

(c) 04:38:01
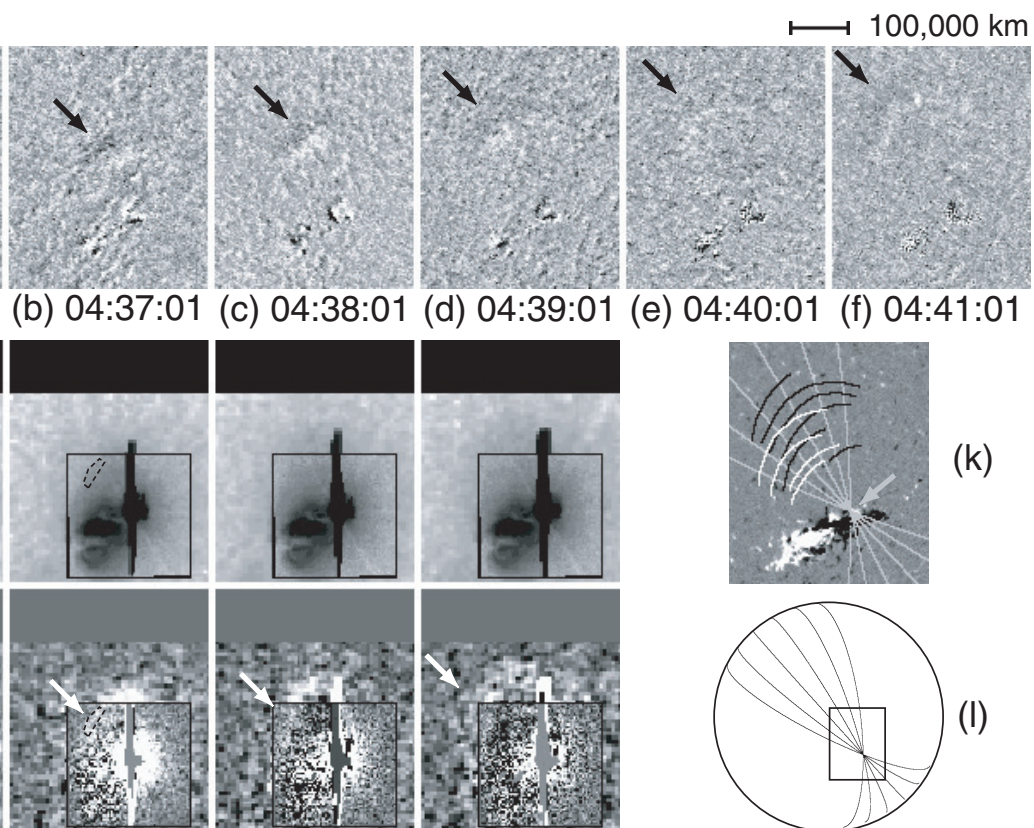

(d) 04:39:01

(e) $04: 40: 01$ (f) $04: 41: 01$
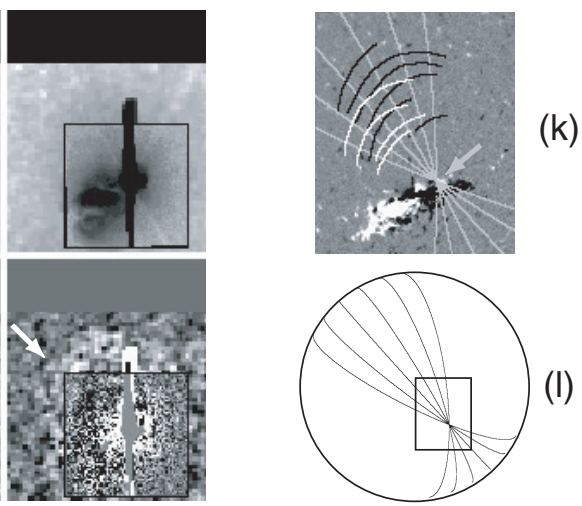

(i) 04:37:00 (j) 04:37:48

(k)

(l)

Figure 45: Structure and evolution of a Moreton wave in soft X-rays and H $\alpha$ observed on 1997 November 3. (a-f) $\mathrm{H} \alpha+0.8 \AA$ running difference images of a Moreton wave (black arrows). (g-j) Soft X-ray (middle panels) and running difference (bottom panels) images of an X-ray wave (white arrows). (k) Wave fronts of the Moreton wave at every minute (black lines) and the X-ray wave at every 48 seconds (white lines) overlaid on the photospheric magnetic field. Gray lines show the great circles through the flare site (gray arrow). The rectangle, circle, and lines shown in (l) are the field of view of $(\mathrm{a}-\mathrm{k})$, the limb of the Sun, and the great circles, respectively (from Narukage et al., 2002).

The so-called EIT wave gives another type of waves associated with a flare, which was discovered by the EUV Imaging Telescope (EIT) aboard SOHO as a transient wave-like phenomenon with enhanced coronal emissions (Moses et al., 1997; Thompson et al., 1998). The mechanism for producing an EIT wave has been investigated by Delannée and Aulanier (1999), Wang (2000), and Wu et al. (2001). Chen et al. (2002) presents an integrated model of EIT and Moreton waves in which these two waves are subproducts of an expanding magnetic loop responsible for a CME.

\subsection{Particle acceleration}

Particle acceleration associated with a flare has intensively been investigated (Ramaty and Murphy, 1987; Miller et al., 1997; Tsuneta and Naito, 1998; Aschwanden, 2002). Detailed processes of particle acceleration are beyond the scope of MHD, where the kinetic process involving charged particles become important. A typical length scale characterizing the kinetic process is the ion Larmor radius or ion inertial length, both of which are of the order meter in the corona. This is much smaller than the typical size of a flare, making it difficult to incorporate particle acceleration

Living Reviews in Solar Physics

http://www . livingreviews . org/lrsp-2011-6 
into the model developed for the global structure and overall evolution of a flare.

Here, we estimate the convective electric field associated with magnetic reconnection, which possibly plays an important role in accelerating charged particles during a flare:

$$
\begin{aligned}
E & \sim \frac{v_{i}}{c} B_{i} \sim \frac{v_{\text {jet }}}{c} B_{\text {jet }} \\
& \sim 3 \times 10^{3}\left(\frac{M_{A}}{0.1}\right)\left(\frac{B}{100 \mathrm{G}}\right)^{2}\left(\frac{n_{\text {jet }}}{10^{10} \mathrm{~cm}^{-3}}\right)^{-1 / 2} \mathrm{~V} \mathrm{~m}^{-1} .
\end{aligned}
$$

This electric field decreases significantly in a gradual phase of a flare because of reduced $v_{i}$, which explains why particle acceleration is less efficient in a gradual phase than an impulsive phase. This also explains why LDE flares have only a gradual phase and not an impulsive phase in which the signature of particle acceleration such as strong HXR emissions is observed.

High-energy electrons produced by strong convective electric field could contribute to forming foot-point as well as loop-top HXR sources, as illustrated in Figure 42. Some of the high-energy electrons are not trapped near the Sun; instead they travel outward through the corona along open field lines. These electrons drive the plasma oscillation in the corona, which is observed as the type III radio bursts.

In some big flares (GOES X-class flares), solar neutron events (SNE) can be observed on the ground of the Earth. The neutrons are produced by the interaction of relativistic ions accelerated at the flare site and atomic nuclei (Watanabe et al., 2006). It is to be noted that when a large amount of electrons are accelerated simultaneously to produce a strong electron beam, a return current (a reverse current) may be generated around the electron beam to cause atmospheric heating. The effect of the return current have been studied by many authors (e.g., Karlický, 2008).

\section{Application to Stellar Flares}

It is well known that stellar flares and coronae have many similarities to solar flares and corona (e.g., Haisch, 1989; Güdel, 2002). Not only light curves of stellar flare emissions (in radio, $\mathrm{H} \alpha$, visible continuum, and X-rays) but also quantitative nature of flares such as time scale, plasma temperature, density, and magnetic field strength are all similar, though the distribution of temperature and total energy of stellar flares is much broader $\left(T \sim 10^{7}-10^{8} \mathrm{~K}\right.$, total energy $10^{29}-10^{37} \mathrm{erg}$ ) than those of solar flares $\left(T \sim 1-3 \times 10^{7} \mathrm{~K}\right.$, total energy $\left.\sim 10^{29}-10^{32} \mathrm{erg}\right)$.

It is believed that stellar flares are produced by the same mechanism, magnetic reconnection, as solar flares. However, why do some of stellar flares show very high temperature and extremely large total energy? Recent observations of young stars by X-ray satellites ASCA and ROSAT have revealed that young stars such as protostars and T-Tauri stars frequently produce superhot flares with temperature of $10^{8} \mathrm{~K}$ (Koyama et al., 1996; Tsuboi et al., 1998; Imanishi et al., 2001, see Feigelson and Montmerle, 1999 for a review). Time variation of X-ray intensity is similar to that of solar flares, while the total energy released by those stellar flares amounts to $10^{36}-10^{37} \mathrm{erg}$, much larger than those of solar flares. Can these protostellar flares be explained on the basis of magnetic reconnection?

A hint was given in a paper by Feldman et al. (1995). They show that there is a universal correlation between flare temperature (T) and emission measure (EM) not only for solar flares but also for some of stellar flares. Shibata and Yokoyama (1999) extended this universal correlation between T and EM and apply it for solar microflares, T-Tauri star flares, and protostellar flares (see Figure 46). It is remarkable that the correlation holds in a very wide range, $4 \times 10^{6} \mathrm{~K}<T<10^{8} \mathrm{~K}$ and $10^{45} \mathrm{~cm}^{-3}<E M<10^{56} \mathrm{~cm}^{-3}$. Shibata and Yokoyama (1999) then found that this universal correlation can be explained by the simple scaling law (see Figure 46),

$$
E M \simeq 10^{48} \mathrm{~cm}^{-3}\left(\frac{B}{50 \mathrm{G}}\right)^{-5}\left(\frac{n_{0}}{10^{9} \mathrm{~cm}^{-3}}\right)^{3 / 2}\left(\frac{T}{10^{7} \mathrm{~K}}\right)^{17 / 2},
$$


which is derived from the following three equations:

$$
\begin{gathered}
E M=n^{2} L^{3}, \\
2 n k T=B^{2} /(8 \pi), \\
T=10^{7} \mathrm{~K}\left(\frac{B}{50 \mathrm{G}}\right)^{6 / 7}\left(\frac{n_{0}}{10^{9} \mathrm{~cm}^{-3}}\right)^{-1 / 7}\left(\frac{L}{10^{9} \mathrm{~K}}\right)^{2 / 7},
\end{gathered}
$$

where $B$ is the magnetic field strength and $n_{0}$ is coronal density, both of which are in a normal state (no occurrence of a flare), $L$ is the length of a flaring loop. Equation (46) is basically the same as Equation (38). ${ }^{1}$

Figure 46 shows the observed correlation between the emission measure of solar and stellar flares and their temperatures. It also shows the theoretical relation between the emission measure and temperature given by the Equation (43) is plotted as solid lines for three cases of $B=15,50,150 \mathrm{G}$ in the case of $n_{0}=10^{9} \mathrm{~cm}^{-3}$. Figure 46 shows that the observed correlation line corresponds to the line of constant magnetic field strength within 30-150 G, and indeed the coronal magnetic field strength is estimated to be about $40-300 \mathrm{G}$ for solar and stellar flares. Similarly, if we eliminate the magnetic field strength $(B)$ from the Equations (43) and (46), we can plot the relation between the emission measure and temperature for constant loop length, which is also shown in Figure 46 as dash-dotted lines. We can see that the length of a solar microflaring loop is $10^{8}-10^{9} \mathrm{~cm}$, and the length of a solar flaring loop is $10^{9}-10^{10} \mathrm{~cm}$. These are fully consistent with observations.

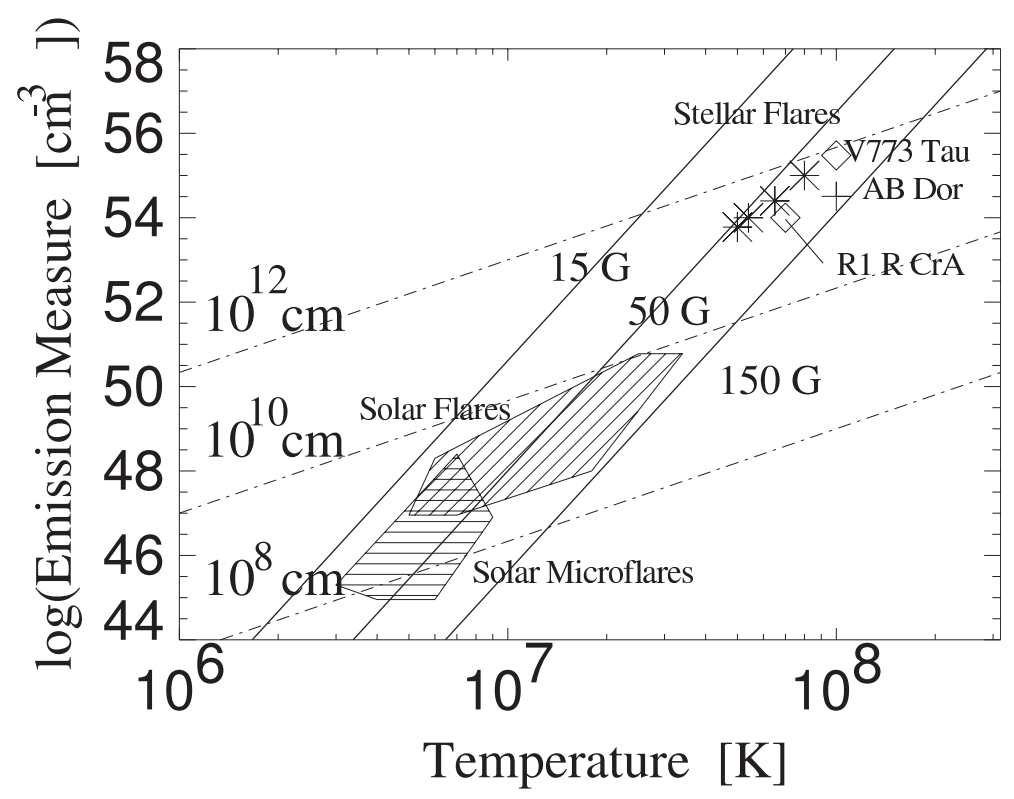

Figure 46: The universal correlation between emission measure and temperature of solar and stellar flares (Shibata and Yokoyama, 1999). The solid lines show the theoretical scaling law $E M \propto B^{-5} T^{17 / 2}$ (Equation (43)) for $B=$ constant $=15,50,150 \mathrm{G}$, and the dash-dotted lines show EM-T relation for $L=$ constant $=10^{8}, 10^{10}, 10^{12} \mathrm{~cm}$.

It is interesting to see that the length of a stellar flaring loop is $10^{10}-10^{12} \mathrm{~cm}$, much larger than the length of a solar flaring loop. This is consistent with observations that average field strength

\footnotetext{
${ }^{1}$ Note that the flare temperature $T$ is a bit cooler than the maximum temperature shown in Equation (38) because of the cooling due to loop's shrinking and evaporation. The reduction rate is roughly estimated as $T \simeq(1 / 3) T_{\text {loop }}$ (Shibata and Yokoyama, 1999).
} 
at the surface of young stars is very strong, which is of order kilo Gauss (e.g., Johns-Krull et al., 1999), indicating that the size of a coronal loop with strong magnetic field ( $\gg 100 \mathrm{G}$ ) is much larger than that in the Sun.

The size of a flaring loop in young stars is estimated to be comparable to or even larger than the solar radius $\left(\sim 7 \times 10^{10} \mathrm{~cm}\right)$. It should be noted here that the range of radius for these young stars is from 1 to 4 solar radii (e.g., Johns-Krull et al., 1999).

Consequently, we found the reason why some of stellar flares, especially young star flares, show very high temperature and extremely large total energy, which is because the size of these flares is much larger than that of solar flares. If the length of a flaring loop is larger, the flare temperature increases in proportion to $L^{2 / 7}$ even if the magnetic field is the same, because the conduction cooling $\left(\kappa_{0} T^{7 / 2} / L^{2}\right)$ become less efficient for a longer loop. The total energy is simply determined by the total magnetic energy contained in the corona in a normal state, $\sim L^{3} B^{2} /(8 \pi)$, which explains the observations very well, although only a fraction of this energy is available as we mentioned before (Equation (1)).

Why can such a large coronal loop with strong magnetic field exist? Why is the filling factor of strong magnetic fields large (near unity) in young stars? One possibility is that the protostar is just born, keeping primordial magnetic field whose origin is in interstellar medium. The other possibility is that the strong magnetic field with large filling factor is created by the dynamo action. Since young stars rotate rapidly (more than $30 \mathrm{~km} \mathrm{~s}^{-1}$ which is much faster than the solar rotation, $\sim 2 \mathrm{~km} \mathrm{~s}^{-1}$ ), the dynamo action would be stronger. It is also expected that there is an accretion disk (planet-forming disk) around a young star, so that strong interaction would occur between the central stellar core and the surrounding disk, which may lead to magnetic reconnection. This interacting process has been treated by Hayashi et al. (1996), who performed 2.5-dimensional MHD simulations for the interaction of an accretion disk and stellar magnetosphere (dipole magnetic field). They have shown that vigous magnetic reconnection associated with mass ejection occurs. The reconnection releases a huge amount of magnetic energy up to the order $10^{36} \mathrm{erg}$ (about $10^{4}$ times more energetic than solar flares) stored in a sheared loop with a size of $L \sim 10^{11} \mathrm{~cm}$.

\section{Concluding Remarks}

As we have seen in the previous sections, the main process responsible for producing a flare is the dissipation of electric current in the corona. The magnetic energy stored by coronal field is first released, followed by various dynamic events such as mass ejection and wave propagation. It should be noticed that the low density in the corona makes these accompanying events so dynamic, which is why we feel that flares are dynamic phenomena as well.

Since the electric conductivity is generally high in the corona, the dissipation of electric current there is only efficient in a current sheet where a large amount of electric current flows. The high electric conductivity of the corona therefore contributes to locally concentrating electric current (this means to amplify free magnetic energy), which in fact makes the dissipation of electric current explosive. This gives an explosive character to a flare, which is quite different from the dissipation of electric current in a resistive medium (conductivity is low) where electric current is dissipated easily and less dynamically without amplifying free magnetic energy.

Here, we summarize the evolution of emerging magnetic field toward the onset of a flare (see also Figure 22). Initially, a magnetic structure is formed on the Sun via flux emergence accompanied by shear and/or converging flows at the surface. Outer marginal part of the structure expands by dominant magnetic pressure force, while inner central part keeps the quasi-static (or slow rise) state where either field-aligned current is dominant (force-free state) or the upward Lorentz force is balanced by the gravitational force exerted on a massive object formed at the inner part (e.g., filament). In either case the free magnetic energy is stored mainly at the inner part, while the outer 
part continuously loses magnetic energy via expansion. If the force balance at the inner part is lost by an instability or loss-of-equilibrium process, part of the free energy is converted into the kinetic energy, producing mass ejection such as filament eruption and CME. This leads to the formation of a current sheet below an ejecting part of the structure which may assume a flux-rope. Magnetic reconnection then occurs in this current sheet to convert remaining free energy into non-magnetic energy, which is observed as a flare. Also the electric field associated with magnetic reconnection produces high-energy particles. Figure 47 shows possible physical processes for producing a flare and accompanying events.

Finally, we should mention that the solar activity including flares potentially has a significant impact on the Earth. This research field investigating the Sun-Earth environment has been developing as the space weather. Flares can produce high-energy particles and CMEs, which sometimes damage telecommunications and power supplies on the Earth. A big flare known as a proton flare produces high-energy protons $(>10 \mathrm{MeV})$, and these high-energy particles travel through the interplanetary space to the Earth, having a huge impact on the polar region of the Earth (polar cap absorption, PCA). Predicting the occurrence of flares therefore becomes of great importance nowadays when human activity extends to the space. This requires the detailed investigations into the mechanism of such magnetically driven solar activity, and the nature of magnetic field transported via flux emergence into the solar atmosphere is a key to a better understanding of the Sun-Earth system.

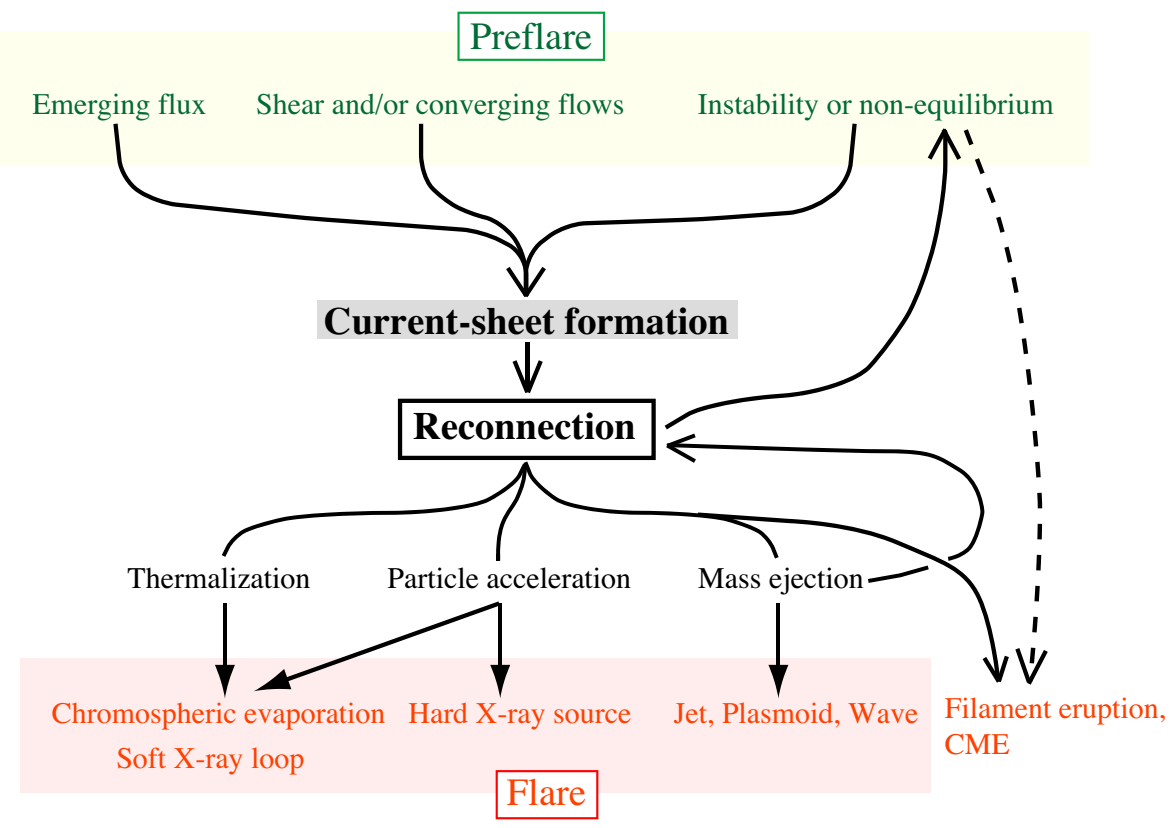

Figure 47: Physical processes responsible for flare and flare-associated phenomena. 


\section{Acknowledgements}

The authors would like to thank the following people for allowing them to use their original figures in this review paper: T. Amari, S. K. Antiochos, V. Archontis, A. Asai, H. Carmichael, P. F. Chen, G. S. Choe, Y. Fan, T. G. Forbes, K. Galsgaard, E. Hiei, T. Hirayama, H. Isobe, S. R. Kane, R. A. Kopp, H. Kurokawa, K. Kusano, J. A. Linker, B.C. Low, W. B. Manchester, S. Masuda, A. McAllister, Z. Mikić, R. L. Moore, F. Moreno-Insertis, N. Narukage, M. Ohyama, E. Pariat, M. Shimojo, P. A. Sturrock, T. Tajima, T. Yokoyama, S. Tsuneta, and A. A. van Ballegooijen.

All figures from the Astrophysical Journal reproduced by permission of the AAS; from Astronomy and Astrophysics reproduced with permission from ESO; from Journal of Geophysical Research reproduced with permission from AGU; from Nature reprinted by permission from Macmillan Publishers Ltd.; from Physics of Plasmas reprinted with permission from AIP; from Publications of the Astronomical Society of Japan reproduced with permission from ASJ; from Solar Physics, Space Science Reviews, and Astrophysics and Space Science reproduced with kind permission from Springer Science+Business Media B.V.

This work was supported by the Grant-in-Aid for the Global COE Program 'The Next Generation of Physics, Spun from Universality and Emergence' from the Ministry of Education, Culture, Sports, Science, and Technology (MEXT) of Japan. One of the authors (T.M.) was also financially supported by Basic Science Research Program (2010-0009258, PI: T. Magara) through the National Research Foundation of Korea (NRF) funded by the Ministry of Education, Science, and Technology, as well as the World Class University (WCU) program through the NRF (R31-10016). 


\section{References}

Abbett, W.P., 2007, "The Magnetic Connection between the Convection Zone and Corona in the Quiet Sun", Astrophys. J., 665, 1469-1488. [DOI], [ADS] (Cited on page 30.)

Abbett, W.P. and Fisher, G.H., 2003, "A Coupled Model for the Emergence of Active Region Magnetic Flux into the Solar Corona", Astrophys. J., 582, 475-485. [DOI], [ADS] (Cited on page 29.)

Acheson, D.J., 1979, "Instability by magnetic buoyancy", Solar Phys., 62, 23-50. [DOI], [ADS] (Cited on page 28.)

Akasofu, S.-I., 1984, "An essay on sunspots and solar flares", Planet. Space Sci., 32, 1469-1471. [DOI], [ADS] (Cited on pages 8 and 21.)

Alfvén, H. and Carlqvist, P., 1967, "Currents in the Solar Atmosphere and a Theory of Solar Flares", Solar Phys., 1, 220-228. [DOI], [ADS] (Cited on pages 8, 15, and 21.)

Aly, J.J., 1984, "On some properties of force-free magnetic fields in infinite regions of space", Astrophys. J., 283, 349-362. [DOI], [ADS] (Cited on page 39.)

Aly, J.J., 1991, "How much energy can be stored in a three-dimensional force-free magnetic field?", Astrophys. J. Lett., 375, L61-L64. [DOI], [ADS] (Cited on page 39.)

Aly, J.J., 1995, "Nonequilibrium in sheared axisymmetric force-free magnetic fields", Astrophys. J. Lett., 439, L63-L66. [DOI], [ADS] (Cited on page 55.)

Amari, T., Luciani, J.F., Aly, J.J. and Tagger, M., 1996, "Very Fast Opening of a Three-dimensional Twisted Magnetic Flux Tube", Astrophys. J. Lett., 466, L39. [DOI], [ADS] (Cited on page 55.)

Amari, T., Luciani, J.F., Mikić, Z. and Linker, J., 2000, "A Twisted Flux Rope Model for Coronal Mass Ejections and Two-Ribbon Flares", Astrophys. J. Lett., 529, L49-L52. [DOI], [ADS] (Cited on pages 57 and 58.)

Amari, T., Luciani, J.F., Aly, J.J., Mikić, Z. and Linker, J., 2003, "Coronal Mass Ejection: Initiation, Magnetic Helicity, and Flux Ropes. I. Boundary Motion-driven Evolution", Astrophys. J., 585, 10731086. [DOI], [ADS] (Cited on page 55.)

Antiochos, S.K., Dahlburg, R.B. and Klimchuk, J.A., 1994, "The magnetic field of solar prominences", Astrophys. J. Lett., 420, L41-L44. [DOI], [ADS] (Cited on page 32.)

Antiochos, S.K., DeVore, C.R. and Klimchuk, J.A., 1999a, "A Model for Solar Coronal Mass Ejections", Astrophys. J., 510, 485-493. [DOI], [ADS], [arXiv:astro-ph/9807220] (Cited on pages 40 and 59.)

Antiochos, S.K., MacNeice, P.J., Spicer, D.S. and Klimchuk, J.A., 1999b, "The Dynamic Formation of Prominence Condensations", Astrophys. J., 512, 985-991. [DOI], [ADS], [arXiv:astro-ph/9808199] (Cited on page 32.)

Antonucci, E., Gabriel, A.H., Acton, L.W., Leibacher, J.W., Culhane, J.L., Rapley, C.G., Doyle, J.G., Machado, M.E. and Orwig, L.E., 1982, "Impulsive phase of flares in soft X-ray emission", Solar Phys., 78, 107-123. [DOI], [ADS] (Cited on page 69.)

Antonucci, E., Gabriel, A.H. and Dennis, B.R., 1984, "The energetics of chromospheric evaporation in solar flares", Astrophys. J., 287, 917-925. [DOI], [ADS] (Cited on page 69.)

Archontis, V., Moreno-Insertis, F., Galsgaard, K., Hood, A. and O'Shea, E., 2004, "Emergence of magnetic flux from the convection zone into the corona", Astron. Astrophys., 426, 1047-1063. [DOI], [ADS] (Cited on pages $28,30,53$, and 55 .) 
Archontis, V., Moreno-Insertis, F., Galsgaard, K. and Hood, A.W., 2005, "The Three-dimensional Interaction between Emerging Magnetic Flux and a Large-Scale Coronal Field: Reconnection, Current Sheets, and Jets", Astrophys. J., 635, 1299-1318. [DOI], [ADS] (Cited on page 30.)

Archontis, V., Hood, A.W. and Brady, C., 2007, "Emergence and interaction of twisted flux tubes in the Sun", Astron. Astrophys., 466, 367-376. [DOI], [ADS] (Cited on page 30.)

Archontis, V., Hood, A.W., Savcheva, A., Golub, L. and DeLuca, E., 2009, "On the Structure and Evolution of Complexity in Sigmoids: A Flux Emergence Model", Astrophys. J., 691, 1276-1291. [DOI], [ADS] (Cited on pages 37 and 38.)

Asai, A., Ishii, T.T., Kurokawa, H., Yokoyama, T. and Shimojo, M., 2003, "Evolution of Conjugate Footpoints inside Flare Ribbons during a Great Two-Ribbon Flare on 2001 April 10", Astrophys. J., 586, 624-629. [DOI], [ADS] (Cited on page 6.)

Asai, A., Yokoyama, T., Shimojo, M. and Shibata, K., 2004, "Downflow Motions Associated with Impulsive Nonthermal Emissions Observed in the 2002 July 23 Solar Flare", Astrophys. J. Lett., 605, L77-L80. [DOI], [ADS] (Cited on page 21.)

Aschwanden, M.J., 2002, "Particle Acceleration and Kinematics in Solar Flares: A Synthesis of Recent Observations and Theoretical Concepts (Invited Review)", Space Sci. Rev., 101, 1-227. [DOI], [ADS] (Cited on pages 10 and 70.)

Aschwanden, M.J., 2004, Physics of the Solar Corona, Springer, Berlin; New York. [Google Books] (Cited on pages 10 and 20.)

Aulanier, G. and Démoulin, P., 1998, "3-D magnetic configurations supporting prominences. I. The natural presence of lateral feet”, Astron. Astrophys., 329, 1125-1137. [ADS] (Cited on page 30.)

Aulanier, G., Démoulin, P., van Driel-Gesztelyi, L., Mein, P. and Deforest, C., 1998, "3-D magnetic configurations supporting prominences. II. The lateral feet as a perturbation of a twisted flux-tube", Astron. Astrophys., 335, 309-322. [ADS] (Cited on page 30.)

Aulanier, G., Pariat, E., Démoulin, P. and Devore, C.R., 2006, "Slip-Running Reconnection in QuasiSeparatrix Layers", Solar Phys., 238, 347-376. [DOI], [ADS] (Cited on page 55.)

Aurass, H., Vršnak, B. and Mann, G., 2002, "Shock-excited radio burst from reconnection outflow jet?", Astron. Astrophys., 384, 273-281. [DOI], [ADS] (Cited on page 69.)

Bak, P., Tang, C. and Wiesenfeld, K., 1987, "Self-Organized Criticality: An Explanation of 1/f Noise", Phys. Rev. Lett., 59, 381-384. [DOI], [ADS] (Cited on page 21.)

Barnes, C.W. and Sturrock, P.A., 1972, "Force-Free Magnetic-Field Structures and Their Role in Solar Activity", Astrophys. J., 174, 659. [DOI], [ADS] (Cited on page 39.)

Bárta, M., Vršnak, B. and Karlický, M., 2008, "Dynamics of plasmoids formed by the current sheet tearing", Astron. Astrophys., 477, 649-655. [DOI], [ADS] (Cited on page 49.)

Bastian, T.S., Benz, A.O. and Gary, D.E., 1998, "Radio Emission from Solar Flares", Annu. Rev. Astron. Astrophys., 36, 131-188. [DOI], [ADS] (Cited on page 10.)

Baty, H., Forbes, T.G. and Priest, E.R., 2009a, "Petschek reconnection with a nonlocalized resistivity", Phys. Plasmas, 16, 012102. [DOI], [ADS] (Cited on page 47.)

Baty, H., Priest, E.R. and Forbes, T.G., 2009b, "Petschek-like reconnection with uniform resistivity", Phys. Plasmas, 16, 060701. [DOI], [ADS] (Cited on page 47.)

Benz, A.O., 1993, Plasma Astrophysics: Kinetic Processes in Solar and Stellar Coronae, vol. 184 of Astrophysics and Space Science Library, Kluwer Academic Publishers, Dordrecht; Boston (Cited on page 10.) 
Berger, M.A. and Field, G.B., 1984, "The topological properties of magnetic helicity", J. Fluid Mech. 147, 133-148. [DOI], [ADS] (Cited on page 40.)

Berger, T.E., Shine, R.A., Slater, G.L., Tarbell, T.D., Title, A.M., Okamoto, T.J., Ichimoto, K., Katsukawa, Y., Suematsu, Y., Tsuneta, S., Lites, B.W. and Shimizu, T., 2008, "Hinode SOT Observations of Solar Quiescent Prominence Dynamics", Astrophys. J. Lett., 676, L89-L92. [DOI], [ADS] (Cited on page 32.)

Bhattacharjee, A., Brunel, F. and Tajima, T., 1983, "Magnetic reconnection driven by the coalescence instability", Phys. Fluids, 26, 3332-3337. [DOI], [ADS] (Cited on page 49.)

Bhattacharjee, A., Ma, Z.W. and Wang, X., 2003, "Recent Developments in Collisionless Reconnection Theory: Applications to Laboratory and Astrophysical Plasmas", in Turbulence and Magnetic Fields in Astrophysics, (Eds.) Falgarone, E., Passot, T., vol. 614 of Lecture Notes in Physics, pp. 351-375, Springer, Berlin. [ADS] (Cited on page 49.)

Birn, J., Drake, J.F., Shay, M.A., Rogers, B.N., Denton, R.E., Hesse, M., Kuznetsova, M., Ma, Z.W., Bhattacharjee, A., Otto, A. and Pritchett, P.L., 2001, "Geospace Environmental Modeling (GEM) magnetic reconnection challenge", J. Geophys. Res., 106, 3715-3720. [DOI], [ADS] (Cited on page 49.)

Biskamp, D., 1986, "Magnetic reconnection via current sheets", Phys. Fluids, 29, 1520-1531. [DOI], [ADS] (Cited on page 47.)

Biskamp, D., 1993, Nonlinear Magnetohydrodynamics, vol. 1 of Cambridge Monographs on Plasma Physics, Cambridge University Press, Cambridge; New York. [ADS] (Cited on page 46.)

Biskamp, D. and Welter, H., 1989, "Magnetic arcade evolution and instability", Solar Phys., 120, 49-77. [DOI], [ADS] (Cited on pages 40, 55, and 56.)

Biskamp, D., Schwarz, E. and Drake, J.F., 1995, "Ion-controlled collisionless magnetic reconnection", Phys. Rev. Lett., 75, 3850-3853. [DOI], [ADS] (Cited on page 49.)

Bruzek, A., 1969, "Motions in Arch Filament Systems", Solar Phys., 8, 29-36. [DOI], [ADS] (Cited on page 23.)

Canfield, R.C., Penn, M.J., Wulser, J.-P. and Kiplinger, A.L., 1990, "H- $\alpha$ spectra of dynamic chromospheric processes in five well-observed X-ray flares", Astrophys. J., 363, 318-325. [DOI], [ADS] (Cited on page 69.)

Canfield, R.C., Hudson, H.S. and McKenzie, D.E., 1999, "Sigmoidal morphology and eruptive solar activity", Geophys. Res. Lett., 26, 627-630. [DOI], [ADS] (Cited on pages 30, 32, and 35.)

Cargill, P.J. and Priest, E.R., 1982, "Slow-shock heating and the Kopp-Pneuman model for 'post'-flare loops", Solar Phys., 76, 357-375. [DOI], [ADS] (Cited on pages 8 and 66.)

Carmichael, H., 1964, "A Process for Flares", in The Physics of Solar Flares, Proceedings of the AASNASA Symposium held 28-30 October, 1963 at the Goddard Space Flight Center, Greenbelt, MD, (Ed.) Hess, W.N., vol. SP-50 of NASA Special Publications, pp. 451-456, NASA Scientific and Technical Information Division, Washington, DC. [ADS] (Cited on pages 7 and 8.)

Carrington, R.C., 1859, "Description of a Singular Appearance seen in the Sun on September 1, 1859", Mon. Not. R. Astron. Soc., 20, 13-15. [ADS] (Cited on page 5.)

Chae, J., 2001, "Observational Determination of the Rate of Magnetic Helicity Transport through the Solar Surface via the Horizontal Motion of Field Line Footpoints", Astrophys. J. Lett., 560, L95-L98. [DOI], [ADS] (Cited on page 44.)

Chae, J., Martin, S.F., Yun, H.S., Kim, J., Lee, S., Goode, P.R., Spirock, T. and Wang, H., 2001, "Small Magnetic Bipoles Emerging in a Filament Channel", Astrophys. J., 548, 497-507. [DOI], [ADS] (Cited on page 30 .) 
Charbonneau, P., McIntosh, S.W., Liu, H.-L. and Bogdan, T.J., 2001, "Avalanche models for solar flares (Invited Review)", Solar Phys., 203, 321-353. [DOI], [ADS] (Cited on pages 10 and 21.)

Chen, J., 1989, "Effects of toroidal forces in current loops embedded in a background plasma", Astrophys. J., 338, 453-470. [DOI], [ADS] (Cited on page 57.)

Chen, P.F., 2011, "Coronal Mass Ejections: Models and Their Observational Basis", Living Rev. Solar Phys., 8, lrsp-2011-1. [ADS]. URL (accessed 30 August 2011): http://www.livingreviews.org/lrsp-2011-1 (Cited on page 10.)

Chen, P.F. and Shibata, K., 2000, "An Emerging Flux Trigger Mechanism for Coronal Mass Ejections", Astrophys. J., 545, 524-531. [DOI], [ADS] (Cited on pages 59 and 60.)

Chen, P.F., Wu, S.T., Shibata, K. and Fang, C., 2002, "Evidence of EIT and Moreton Waves in Numerical Simulations", Astrophys. J. Lett., 572, L99-L102. [DOI], [ADS] (Cited on page 70.)

Cheung, M.C.M., Moreno-Insertis, F. and Schüssler, M., 2006, "Moving magnetic tubes: fragmentation, vortex streets and the limit of the approximation of thin flux tubes", Astron. Astrophys., 451, 303-317. [DOI], $[\mathrm{ADS}]$ (Cited on page 23.)

Cheung, M.C.M., Schüssler, M. and Moreno-Insertis, F., 2007, "Magnetic flux emergence in granular convection: radiative MHD simulations and observational signatures", Astron. Astrophys., 467, 703719. [DOI], [ADS], [arXiv:astro-ph/0702666] (Cited on page 30.)

Cheung, M.C.M., Schüssler, M., Tarbell, T.D. and Title, A.M., 2008, "Solar Surface Emerging Flux Regions: A Comparative Study of Radiative MHD Modeling and Hinode SOT Observations", Astrophys. J., 687, 1373-1387. [DOI], [ADS], [arXiv:0810.5723] (Cited on page 30.)

Choe, G.S. and Cheng, C.Z., 2000, "A Model of Solar Flares and Their Homologous Behavior", Astrophys. J., 541, 449-467. [DOI], [ADS] (Cited on pages 49, 64, and 65.)

Choe, G.S. and Cheng, C.Z., 2002, "Energy of Force-free Magnetic Fields in Relation to Coronal Mass Ejections", Astrophys. J. Lett., 574, L179-L182. [DOI], [ADS] (Cited on page 40.)

Choe, G.S. and Lee, L.C., 1996, "Evolution of Solar Magnetic Arcades. II. Effect of Resistivity and Solar Eruptive Processes", Astrophys. J., 472, 372. [DOI], [ADS] (Cited on pages 55 and 56.)

Chou, D.-Y. and Zirin, H., 1988, "The vertical structure of arch filament systems in solar emerging flux regions", Astrophys. J., 333, 420-426. [DOI], [ADS] (Cited on page 23.)

Cowling, T.G., 1953, "Solar Electrodynamics", in The Solar System, Vol. 1: The Sun, (Ed.) Kuiper, G.P., pp. 532-591, University of Chicago Press, Chicago. [ADS] (Cited on page 5.)

Craig, I.J.D. and Watson, P.G., 2005, "Exact models for Hall current reconnection with axial guide fields", Phys. Plasmas, 12, 012306. [DOI], [ADS] (Cited on page 49.)

de Jager, C. and Sakai, J.-I., 1991, "Elementary flare bursts explained by explosive coalescence", Solar Phys., 133, 395-398. [DOI], [ADS] (Cited on page 61.)

Delannée, C. and Aulanier, G., 1999, "CME Associated with Transequatorial Loops and a Bald Patch Flare", Solar Phys., 190, 107-129. [DOI], [ADS] (Cited on page 70.)

Démoulin, P. and Aulanier, G., 2010, "Criteria for Flux Rope Eruption: Non-equilibrium Versus Torus Instability", Astrophys. J., 718, 1388-1399. [DOI], [ADS], [arXiv:1006.1785 [astro-ph.SR]] (Cited on page 57.)

Démoulin, P. and Berger, M.A., 2003, "Magnetic Energy and Helicity Fluxes at the Photospheric Level", Solar Phys., 215, 203-215. [ADS] (Cited on page 44.) 
Démoulin, P. and Pariat, E., 2009, "Modelling and observations of photospheric magnetic helicity", Adv. Space Res., 43, 1013-1031. [DOI], [ADS] (Cited on page 44.)

Démoulin, P., Henoux, J.C., Priest, E.R. and Mandrini, C.H., 1996, "Quasi-Separatrix layers in solar flares. I. Method", Astron. Astrophys., 308, 643-655. [ADS] (Cited on page 55.)

Dennis, B.R., 1985, "Solar hard X-ray bursts", Solar Phys., 100, 465-490. [DOI], [ADS] (Cited on pages 10 and 20.)

DeVore, C.R., 2000, "Magnetic Helicity Generation by Solar Differential Rotation", Astrophys. J., 539, 944-953. [DOI], [ADS] (Cited on page 40.)

DeVore, C.R. and Antiochos, S.K., 2000, "Dynamical Formation and Stability of Helical Prominence Magnetic Fields", Astrophys. J., 539, 954-963. [DOI], [ADS] (Cited on pages 31, 32, and 55.)

Drake, J.F., Swisdak, M., Cattell, C., Shay, M.A., Rogers, B.N. and Zeiler, A., 2003, "Formation of Electron Holes and Particle Energization During Magnetic Reconnection", Science, 299, 873-877. [DOI], [ADS] (Cited on page 49.)

Dulk, G.A., 1985, "Radio emission from the sun and stars", Annu. Rev. Astron. Astrophys., 23, 169-224. [DOI], $[\mathrm{ADS}]$ (Cited on page 10.)

Emonet, T. and Moreno-Insertis, F., 1998, "The Physics of Twisted Magnetic Tubes Rising in a Stratified Medium: Two-dimensional Results", Astrophys. J., 492, 804-821. [DOI], [ADS], [arXiv:astroph/9711043] (Cited on page 23.)

Eto, S., Isobe, H., Narukage, N., Asai, A., Morimoto, T., Thompson, B., Yashiro, S., Wang, T., Kitai, R., Kurokawa, H. and Shibata, K., 2002, "Relation between a Moreton Wave and an EIT Wave Observed on 1997 November 4", Publ. Astron. Soc. Japan, 54, 481-491. [ADS] (Cited on page 70.)

Fan, Y., 2001, "The Emergence of a Twisted $\Omega$-Tube into the Solar Atmosphere", Astrophys. J. Lett., 554, L111-L114. [DOI], [ADS] (Cited on pages 29 and 55.)

Fan, Y., 2008, "The Three-dimensional Evolution of Buoyant Magnetic Flux Tubes in a Model Solar Convective Envelope", Astrophys. J., 676, 680-697. [DOI], [ADS] (Cited on page 23.)

Fan, Y., 2009, "Magnetic Fields in the Solar Convection Zone", Living Rev. Solar Phys., 6, lrsp-2009-4. [ADS]. URL (accessed 1 November 2011):

http://www.livingreviews.org/lrsp-2009-4 (Cited on page 8.)

Fan, Y. and Gibson, S.E., 2003, "The Emergence of a Twisted Magnetic Flux Tube into a Preexisting Coronal Arcade", Astrophys. J. Lett., 589, L105-L108. [DOI], [ADS] (Cited on pages 57 and 58.)

Feigelson, E.D. and Montmerle, T., 1999, "High-Energy Processes in Young Stellar Objects", Annu. Rev. Astron. Astrophys., 37, 363-408. [DOI], [ADS] (Cited on page 71.)

Feldman, U., Laming, J.M. and Doschek, G.A., 1995, "The Correlation of Solar Flare Temperature and Emission Measure Extrapolated to the Case of Stellar Flares", Astrophys. J. Lett., 451, L79. [DOI], [ADS] (Cited on page 71.)

Feynman, J. and Martin, S.F., 1995, "The initiation of coronal mass ejections by newly emerging magnetic flux", J. Geophys. Res., 100, 3355-3367. [DOI], [ADS] (Cited on page 9.)

Finn, J.M. and Antonsen Jr, T.M., 1985, "Magnetic helicity: what is it, and what is it good for?", Comments Plasma Phys. Contr. Fusion, 9, 111-126 (Cited on page 40.)

Fisher, G.H. and Hawley, S.L., 1990, "An equation for the evolution of solar and stellar flare loops", Astrophys. J., 357, 243-258. [DOI], [ADS] (Cited on pages 68 and 69.) 
Forbes, T.G., 2000, "A review on the genesis of coronal mass ejections", J. Geophys. Res., 105, 23,15323,166. [DOI], [ADS] (Cited on page 10.)

Forbes, T.G. and Acton, L.W., 1996, "Reconnection and Field Line Shrinkage in Solar Flares", Astrophys. $J ., 459,330$. [DOI], [ADS] (Cited on pages 12 and 21.)

Forbes, T.G. and Isenberg, P.A., 1991, "A catastrophe mechanism for coronal mass ejections", Astrophys. J., 373, 294-307. [DOI], [ADS] (Cited on pages 57 and 58.)

Forbes, T.G. and Malherbe, J.M., 1986, "A shock condensation mechanism for loop prominences", Astrophys. J. Lett., 302, L67-L70. [DOI], [ADS] (Cited on pages 8 and 66.)

Forbes, T.G. and Malherbe, J.M., 1991, "A numerical simulation of magnetic reconnection and radiative cooling in line-tied current sheets", Solar Phys., 135, 361-391. [DOI], [ADS] (Cited on page 12.)

Forbes, T.G. and Priest, E.R., 1983, "A numerical experiment relevant to line-tied reconnection in tworibbon flares", Solar Phys., 84, 169-188. [DOI], [ADS] (Cited on page 69.)

Forbes, T.G. and Priest, E.R., 1984, "Numerical simulation of reconnection in an emerging magnetic flux region", Solar Phys., 94, 315-340. [DOI], [ADS] (Cited on pages 8 and 50.)

Forbes, T.G. and Priest, E.R., 1987, "A comparison of analytical and numerical models for steadily driven magnetic reconnection", Rev. Geophys., 25, 1583-1607. [DOI], [ADS] (Cited on page 46.)

Forbes, T.G., Malherbe, J.M. and Priest, E.R., 1989, "The formation flare loops by magnetic reconnection and chromospheric ablation", Solar Phys., 120, 285-307. [DOI], [ADS] (Cited on pages 66 and 68.)

Forbes, T.G., Linker, J.A., Chen, J., Cid, C., Kóta, J., Lee, M.A., Mann, G., Mikić, Z., Potgieter, M.S., Schmidt, J.M., Siscoe, G.L., Vainio, R., Antiochos, S.K. and Riley, P., 2006, "CME Theory and Models", Space Sci. Rev., 123, 251-302. [DOI], [ADS] (Cited on page 10.)

Furth, H.P., Killeen, J. and Rosenbluth, M.N., 1963, "Finite-Resistivity Instabilities of a Sheet Pinch", Phys. Fluids, 6, 459-484. [DOI] (Cited on page 49.)

Galsgaard, K., Moreno-Insertis, F., Archontis, V. and Hood, A., 2005, "A Three-dimensional Study of Reconnection, Current Sheets, and Jets Resulting from Magnetic Flux Emergence in the Sun", Astrophys. J. Lett., 618, L153-L156. [DOI], [ADS], [arXiv:astro-ph/0410057] (Cited on page 30.)

Galsgaard, K., Archontis, V., Moreno-Insertis, F. and Hood, A.W., 2007, "The Effect of the Relative Orientation between the Coronal Field and New Emerging Flux. I. Global Properties", Astrophys. J., 666, 516-531. [DOI], [ADS], [arXiv:0705.1097] (Cited on pages 30, 54, and 55.)

Gan, W.Q., Zhang, H.Q. and Fang, C., 1991, "A hydrodynamic model of the impulsive phase of a solar flare loop", Astron. Astrophys., 241, 618-624. [ADS] (Cited on page 69.)

Gibson, S.E., Fletcher, L., Del Zanna, G., Pike, C.D., Mason, H.E., Mandrini, C.H., Démoulin, P., Gilbert, H., Burkepile, J., Holzer, T., Alexander, D., Liu, Y., Nitta, N., Qiu, J., Schmieder, B. and Thompson, B.J., 2002, "The Structure and Evolution of a Sigmoidal Active Region", Astrophys. J., 574, 1021-1038. [DOI], $[\mathrm{ADS}]$ (Cited on page 32.)

Gibson, S.E., Fan, Y., Török, T. and Kliem, B., 2006, "The Evolving Sigmoid: Evidence for Magnetic Flux Ropes in the Corona Before, During, and After CMEs", Space Sci. Rev., 124, 131-144. [DOI], [ADS] (Cited on page 10.)

Giovanelli, R.G., 1946, "A Theory of Chromospheric Flares", Nature, 158, 81-82. [DOI], [ADS] (Cited on page 5.)

Gold, T. and Hoyle, F., 1960, "On the origin of solar flares", Mon. Not. R. Astron. Soc., 120, 89. [ADS] (Cited on pages 17 and 23.) 
Golub, L. and Pasachoff, J.M., 1997, The Solar Corona, Cambridge University Press, Cambridge. [ADS], [Google Books] (Cited on page 10.)

Gosling, J.T., 1997, "Coronal Mass Ejections: An Overview", in Coronal Mass Ejections, (Eds.) Crooker, N., Joselyn, J.A., Feynman, J., vol. 99 of Geophysical Monograph, pp. 9-16, American Geophysical Union, Washington, DC (Cited on page 10.)

Güdel, M., 2002, "Stellar Radio Astronomy: Probing Stellar Atmospheres from Protostars to Giants", Annu. Rev. Astron. Astrophys., 40, 217-261. [DOI], [ADS], [arXiv:astro-ph/0206436] (Cited on page 71.)

Haisch, B., Strong, K.T. and Rodono, M., 1991, "Flares on the sun and other stars", Annu. Rev. Astron. Astrophys., 29, 275-324. [DOI], [ADS] (Cited on page 10.)

Haisch, B.M., 1989, “An overview of solar and stellar flare research", Solar Phys., 121, 3-18. [DOI], [ADS] (Cited on page 71.)

Hale, G.E., 1908, "On the Probable Existence of a Magnetic Field in Sun-Spots", Astrophys. J., 28, 315-343. [DOI], [ADS] (Cited on page 5.)

Hanaoka, Y., Kurokawa, H. and Saito, S., 1986, "The POST flare loops observed at the total eclipse of February 16, 1980", Solar Phys., 105, 133-148. [DOI], [ADS] (Cited on page 12.)

Hanaoka, Y., Kurokawa, H., Enome, S., Nakajima, H., Shibasaki, K., Nishio, M., Takano, T., Torii, C., Sekiguchi, H., Kawashima, S., Bushimata, T., Shinohara, N., Irimajiri, Y., Koshiishi, H., Shiomi, Y., Nakai, Y., Funakoshi, Y., Kitai, R., Ishiura, K. and Kimura, G., 1994, "Simultaneous Observations of a Prominence Eruption Followed by a Coronal Arcade Formation in Radio, Soft X-Rays, and H $\alpha$ ", Publ. Astron. Soc. Japan, 46, 205-216. [ADS] (Cited on page 14.)

Hanasz, M. and Lesch, H., 2003, "Conditions for fast magnetic reconnection in astrophysical plasmas", Astron. Astrophys., 404, 389-395. [DOI], [ADS], [arXiv:astro-ph/0303608] (Cited on page 49.)

Hansteen, V.H., de Pontieu, B., Carlsson, M., McIntosh, S., Watanabe, T., Warren, H.P., Harra, L.K., Hara, H., Tarbell, T.D., Shine, D., Title, A.M., Schrijver, C.J., Tsuneta, S., Katsukawa, Y., Ichimoto, K., Suematsu, Y. and Shimizu, T., 2007, "On Connecting the Dynamics of the Chromosphere and Transition Region with Hinode SOT and EIS", Publ. Astron. Soc. Japan, 59, 699. [ADS], [arXiv:0711.0487] (Cited on page 30.)

Hara, H. and Ichimoto, K., 1997, "A Coronal Velocity Field around a Long-Duration Event: Search for Reconnection Inflow", in Magnetic Reconnection in the Solar Atmosphere, (Eds.) Bentley, R.D., Mariska, J.T., vol. 111 of ASP Conference Series, p. 183, Astronomical Society of the Pacific, San Francisco. [ADS] (Cited on page 46.)

Hara, H., Nishino, Y., Ichimoto, K. and Delaboudinière, J.-P., 2006, "A Spectroscopic Observation of a Magnetic Reconnection Site in a Small Flaring Event", Astrophys. J., 648, 712-721. [DOI], [ADS] (Cited on pages 21 and 46.)

Harra, L.K. and Sterling, A.C., 2001, "Material Outflows from Coronal Intensity 'Dimming Regions' during Coronal Mass Ejection Onset", Astrophys. J. Lett., 561, L215-L218. [DOI], [ADS] (Cited on page 15.)

Hayashi, M.R., Shibata, K. and Matsumoto, R., 1996, "X-Ray Flares and Mass Outflows Driven by Magnetic Interaction between a Protostar and Its Surrounding Disk", Astrophys. J. Lett., 468, L37L40. [DOI], [ADS], [arXiv:astro-ph/9606157] (Cited on page 73.)

Heitsch, F. and Zweibel, E.G., 2003, "Fast Reconnection in a Two-Stage Process", Astrophys. J., 583, 229-244. [DOI], [ADS], [arXiv:astro-ph/0205103] (Cited on page 49.)

Heyvaerts, J., Priest, E.R. and Rust, D.M., 1977, "An emerging flux model for the solar flare phenomenon", Astrophys. J., 216, 123-137. [DOI], [ADS] (Cited on pages 9 and 50.) 
Hiei, E., Hundhausen, A.J. and Sime, D.G., 1993, "Reformation of a coronal helmet streamer by magnetic reconnection after a coronal mass ejection", Geophys. Res. Lett., 20, 2785-2788. [DOI], [ADS] (Cited on pages 14 and 15.)

Hirayama, T., 1974, "Theoretical Model of Flares and Prominences. I: Evaporating Flare Model", Solar Phys., 34, 323-338. [DOI], [ADS] (Cited on pages 7, 8, and 68.)

Hodgson, R., 1859, "On a curious Appearance seen in the Sun", Mon. Not. R. Astron. Soc., 20, 15-16. [ADS] (Cited on page 5.)

Hood, A.W., Archontis, V., Galsgaard, K. and Moreno-Insertis, F., 2009, "The emergence of toroidal flux tubes from beneath the solar photosphere", Astron. Astrophys., 503, 999-1011. [DOI], [ADS] (Cited on page 30.)

Hori, K., Yokoyama, T., Kosugi, T. and Shibata, K., 1997, "Pseudo-Two-dimensional Hydrodynamic Modeling of Solar Flare Loops", Astrophys. J., 489, 426-441. [DOI], [ADS] (Cited on page 69.)

Horiuchi, R. and Sato, T., 1999, "Collisionless magnetic reconnection in the presence of an external driving flow", J. Plasma Phys., 61, 415-423. [DOI], [ADS] (Cited on page 49.)

Horton, W. and Tajima, T., 1988, "Linear theory of driven reconnection", J. Geophys. Res., 93, 2741-2748. [DOI], $[\mathrm{ADS}]$ (Cited on page 49.)

Hoyle, F., 1949, Some Recent Researches in Solar Physics, Cambridge Monographs on Physics, Cambridge University Press, Cambridge. [ADS] (Cited on page 5.)

Hudson, H.S., 1991, "Solar flares, microflares, nanoflares, and coronal heating", Solar Phys., 133, 357-369. [DOI], [ADS] (Cited on pages 17 and 20.)

Hudson, H.S., 1994, "Thermal Plasmas in the Solar Corona: The Yohkoh Soft X-Ray Observations", in Proceedings of Kofu Symposium 'New Look at the Sun with Emphasis on Advanced Observations of Coronal Dynamics and Flares', Kofu, Japan, September 6-10, 1993, (Eds.) Enome, S., Hirayama, T., vol. 360 of NRO Report, pp. 1-10, Nobeyama Radio Observatory, Nobeyama. [ADS]. Online version (accessed 10 December 2009):

http://solar.nro.nao.ac.jp/meeting/kofu/ (Cited on pages 12 and 21.)

Hudson, H.S. and Ryan, J., 1995, "High-Energy Particles in Solar Flares", Annu. Rev. Astron. Astrophys., 33, 239-282. [DOI], [ADS] (Cited on page 10.)

Hundhausen, J.R., 1999, "Coronal Mass Ejections", in The Many Faces of the Sun: A Summary of the Results from NASA's Solar Maximum Mission, (Eds.) Strong, K.T., Saba, J.L.R., Haisch, B.M., Schmelz, J.T., p. 143, Springer, New York. [ADS] (Cited on page 10.)

Ichimoto, K. and Kurokawa, H., 1984, "H-alpha red asymmetry of solar flares", Solar Phys., 93, $105-121$. [DOI], [ADS] (Cited on page 69.)

Imanishi, K., Koyama, K. and Tsuboi, Y., 2001, "Chandra Observation of the $\rho$ Ophiuchi Cloud", Astrophys. J., 557, 747-760. [DOI], [ADS] (Cited on page 71.)

Inhester, B., Birn, J. and Hesse, M., 1992, "The evolution of line-tied coronal arcades including a converging footpoint motion", Solar Phys., 138, 257-281. [DOI], [ADS] (Cited on page 55.)

Innes, D.E., McKenzie, D.E. and Wang, T., 2003, "SUMER spectral observations of post-flare supra-arcade inflows", Solar Phys., 217, 247-265. [ADS] (Cited on page 21.)

Inoue, S. and Kusano, K., 2006, "Three-dimensional Simulation Study of Flux Rope Dynamics in the Solar Corona", Astrophys. J., 645, 742-756. [DOI], [ADS] (Cited on page 57.)

Isenberg, P.A. and Forbes, T.G., 2007, "A Three-dimensional Line-tied Magnetic Field Model for Solar Eruptions", Astrophys. J., 670, 1453-1466. [DOI], [ADS] (Cited on page 57.) 
Ishii, T.T., Kurokawa, H. and Takeuchi, T.T., 1998, "Emergence of a Twisted Magnetic Flux Bundle as a Source of Strong Flare Activity", Astrophys. J., 499, 898. [DOI], [ADS], [arXiv:astro-ph/9708208] (Cited on page 23.)

Isobe, H., Yokoyama, T., Shimojo, M., Morimoto, T., Kozu, H., Eto, S., Narukage, N. and Shibata, K., 2002, "Reconnection Rate in the Decay Phase of a Long Duration Event Flare on 1997 May 12", Astrophys. J., 566, 528-538. [DOI], [ADS] (Cited on page 46.)

Isobe, H., Miyagoshi, T., Shibata, K. and Yokoyama, T., 2005, "Filamentary structure on the Sun from the magnetic Rayleigh-Taylor instability", Nature, 434, 478-481. [DOI], [ADS] (Cited on pages 30, 52, and 55.)

Isobe, H., Miyagoshi, T., Shibata, K. and Yokoyama, T., 2006, "Three-Dimensional Simulation of Solar Emerging Flux Using the Earth Simulator I. Magnetic Rayleigh-Taylor Instability at the Top of the Emerging Flux as the Origin of Filamentary Structure", Publ. Astron. Soc. Japan, 58, 423-438. [ADS] (Cited on pages 30 and 55.)

Jeong, H. and Chae, J., 2007, "Magnetic Helicity Injection in Active Regions", Astrophys. J., 671, 10221033. [DOI], [ADS] (Cited on page 44.)

Ji, H., Terry, S., Yamada, M., Kulsrud, R., Kuritsyn, A. and Ren, Y., 2004, "Electromagnetic Fluctuations during Fast Reconnection in a Laboratory Plasma", Phys. Rev. Lett., 92, 115001. [DOI], [ADS], [arXiv:astro-ph/0301593] (Cited on page 49.)

Johns-Krull, C.M., Valenti, J.A. and Koresko, C., 1999, "Measuring the Magnetic Field on the Classical T Tauri Star BP Tauri", Astrophys. J., 516, 900-915. [DOI], [ADS] (Cited on page 73.)

Kahler, S.W., 1992, "Solar flares and coronal mass ejections", Annu. Rev. Astron. Astrophys., 30, $113-141$. [DOI], [ADS] (Cited on page 10.)

Kane, S.R., 1974, "Impulsive (Flash) Phase of Solar Flares: Hard X-Ray, Microwave, EUV and Optical Observations", in Coronal Disturbances, IAU Symposium no. 57, held at Surfers Paradise, Queensland, Australia, 7-11 September, 1973, (Ed.) Newkirk Jr, G., vol. 57 of IAU Symposium, pp. 105-141, Reidel, Dordrecht; Boston. [ADS], [Google Books] (Cited on page 62.)

Karlický, M., 2008, "Return Current Formation in the Region of Escaping Electrons from the Acceleration Space", in 12th European Solar Physics Meeting (ESPM-12), Freiburg, Germany, held September, 8-12, 2008, (Ed.) Peter, H., vol. 12, Kiepenheuer-Institut für Sonnenphysik, Freiburg. [ADS]. URL (accessed 22 September 2011):

http://espm.kis.uni-freiburg.de/ (Cited on page 71.)

Karlický, M. and Bárta, M., 2007, "Drifting pulsating structures generated during tearing and coalescence processes in a flare current sheet", Astron. Astrophys., 464, 735-740. [DOI], [ADS] (Cited on page 49.)

Karlický, M. and Kosugi, T., 2004, "Acceleration and heating processes in a collapsing magnetic trap", Astron. Astrophys., 419, 1159-1168. [DOI], [ADS] (Cited on page 61.)

Karpen, J.T. and Antiochos, S.K., 2008, "Condensation Formation by Impulsive Heating in Prominences", Astrophys. J., 676, 658-671. [DOI], [ADS] (Cited on page 32.)

Khan, J.I. and Hudson, H.S., 2000, "Homologous sudden disappearances of transequatorial interconnecting loops in the solar corona", Geophys. Res. Lett., 27, 1083. [DOI], [ADS] (Cited on page 70.)

Kim, Y.-H., Moon, Y.-J., Cho, K.-S., Bong, S.-C. and Park, Y.D., 2005, "A Study of Flare-associated XRay Plasma Ejections. III. Kinematic Properties", Astrophys. J., 635, 1291-1298. [DOI], [ADS] (Cited on page 21.)

Kliem, B. and Török, T., 2006, "Torus Instability", Phys. Rev. Lett., 96, 255002. [DOI], [ADS], [arXiv:physics/0605217] (Cited on page 57.) 
Klimchuk, J.A., 2001, "Theory of Coronal Mass Ejections", in Space Weather, (Eds.) Song, P., Singer, H.J., Siscoe, H.L., vol. 125 of Geophysical Monograph, pp. 143-157, American Geophysical Union, Washington, DC. [ADS] (Cited on page 10.)

Kopp, R.A. and Pneuman, G.W., 1976, "Magnetic reconnection in the corona and the loop prominence phenomenon", Solar Phys., 50, 85-98. [DOI], [ADS] (Cited on pages 7 and 8.)

Kosugi, T., Sakao, T., Masuda, S., Hara, H., Shimizu, T. and Hudson, H.S., 1994, "Hard and Soft X-ray Observations of a Super-Hot Thermal Flare of 6 February, 1992", in Proceedings of Kofu Symposium 'New Look at the Sun with Emphasis on Advanced Observations of Coronal Dynamics and Flares', Kofu, Japan, September 6-10, 1993, (Eds.) Enome, S., Hirayama, T., vol. 360 of NRO Report, pp. 127-129, Nobeyama Radio Observatory, Nobeyama. [ADS]. Online version (accessed 10 December 2009): http://solar.nro.nao.ac.jp/meeting/kofu/ (Cited on page 68.)

Koyama, K., Hamaguchi, K., Ueno, S., Kobayashi, N. and Feigelson, E.D., 1996, "Discovery of Hard XRays from a Cluster of Protostars", Publ. Astron. Soc. Japan, 48, L87-L92. [ADS] (Cited on page 71.)

Krall, J., Chen, J., Santoro, R., Spicer, D.S., Zalesak, S.T. and Cargill, P.J., 1998, "Simulation of Buoyant Flux Ropes in a Magetized Solar Atmosphere", Astrophys. J., 500, 992. [DOI], [ADS] (Cited on page 25.)

Kulsrud, R.M., 2001, "Magnetic reconnection: Sweet-Parker versus Petschek", Solar Phys., 53, 417-422. [ADS], [arXiv:astro-ph/0007075] (Cited on page 47.)

Kurokawa, H., 1987, "Two distinct morphological types of magnetic shear development and their relation to flares", Solar Phys., 113, 259-263. [DOI], [ADS] (Cited on page 23.)

Kurokawa, H., 1989, "High-resolution observations of H $\alpha$ flare regions", Space Sci. Rev., 51, 49-84. [DOI], [ADS] (Cited on page 24.)

Kusano, K., Suzuki, Y. and Nishikawa, K., 1995, "A solar flare triggering mechanism based on the WoltjerTaylor minimum energy principle", Astrophys. J., 441, 942-951. [DOI], [ADS] (Cited on page 40.)

Kusano, K., Maeshiro, T., Yokoyama, T. and Sakurai, T., 2002, "Measurement of Magnetic Helicity Injection and Free Energy Loading into the Solar Corona", Astrophys. J., 577, 501-512. [DOI], [ADS] (Cited on page 44.)

Kusano, K., Maeshiro, T., Yokoyama, T. and Sakurai, T., 2004, "The Trigger Mechanism of Solar Flares in a Coronal Arcade with Reversed Magnetic Shear", Astrophys. J., 610, 537-549. [DOI], [ADS] (Cited on pages 59 and 60. )

Leake, J.E. and Arber, T.D., 2006, "The emergence of magnetic flux through a partially ionised solar atmosphere", Astron. Astrophys., 450, 805-818. [DOI], [ADS] (Cited on page 30.)

Leka, K.D., Canfield, R.C., McClymont, A.N. and van Driel-Gesztelyi, L., 1996, "Evidence for Currentcarrying Emerging Flux", Astrophys. J., 462, 547-560. [DOI], [ADS] (Cited on page 23.)

Lim, E.-K. and Chae, J., 2009, "Chirality of Intermediate Filaments and Magnetic Helicity of Active Regions", Astrophys. J., 692, 104-108. [DOI], [ADS], [arXiv:0901.0360 [astro-ph.SR]] (Cited on page 41.)

Lin, J., 2004, "CME-Flare Association Deduced from Catastrophic Model of CMEs", Solar Phys., 219, 169-196. [DOI], [ADS] (Cited on page 57.)

Lin, J., Soon, W. and Baliunas, S.L., 2003, "Theories of solar eruptions: a review", New Astron. Rev., 47, 53-84. [DOI], [ADS] (Cited on page 10.)

Lin, J., Ko, Y.-K., Sui, L., Raymond, J.C., Stenborg, G.A., Jiang, Y., Zhao, S. and Mancuso, S., 2005, "Direct Observations of the Magnetic Reconnection Site of an Eruption on 2003 November 18", Astrophys. J., 622, 1251-1264. [DOI], [ADS] (Cited on page 21.) 
Lin, R.P., Schwartz, R.A., Kane, S.R., Pelling, R.M. and Hurley, K.C., 1984, "Solar hard X-ray microflares", Astrophys. J., 283, 421-425. [DOI], [ADS] (Cited on pages 17 and 20.)

Linker, J.A., Mikić, Z., Lionello, R., Riley, P., Amari, T. and Odstrcil, D., 2003, "Flux cancellation and coronal mass ejections", Phys. Plasmas, 10, 1971-1978. [DOI], [ADS] (Cited on pages 57 and 58.)

Linton, M.G. and Longcope, D.W., 2006, "A Model for Patchy Reconnection in Three Dimensions", Astrophys. J., 642, 1177-1192. [DOI], [ADS], [astro-ph/0509348] (Cited on page 21.)

Lites, B.W., 2005, "Magnetic Flux Ropes in the Solar Photosphere: The Vector Magnetic Field under Active Region Filaments", Astrophys. J., 622, 1275-1291. [DOI], [ADS] (Cited on page 32.)

Longcope, D.W., 1998, "A Model for Current Sheets and Reconnection in X-Ray Bright Points", Astrophys. J., 507, 433-442. [DOI], [ADS] (Cited on page 55.)

Longcope, D.W., 2005, "Topological Methods for the Analysis of Solar Magnetic Fields", Living Rev. Solar Phys., 2, lrsp-2005-7. [ADS]. URL (accessed 24 November 2009):

http://www.livingreviews.org/lrsp-2005-7 (Cited on pages 9 and 55.)

Longcope, D.W. and Magara, T., 2004, "A Comparison of the Minimum Current Corona to a Magnetohydrodynamic Simulation of Quasi-Static Coronal Evolution", Astrophys. J., 608, 1106-1123. [DOI], [ADS] (Cited on page 55.)

Longcope, D.W., Fisher, G.H. and Pevtsov, A.A., 1998, "Flux-Tube Twist Resulting from Helical Turbulence: The $\Sigma$-Effect", Astrophys. J., 507, 417-432. [DOI], [ADS] (Cited on page 23.)

López Ariste, A., Aulanier, G., Schmieder, B. and Sainz Dalda, A., 2006, "First observation of bald patches in a filament channel and at a barb endpoint", Astron. Astrophys., 456, 725-735. [DOI] (Cited on page 30.)

Low, B.C., 1977, "Evolving force-free magnetic fields. I. The development of the preflare stage", Astrophys. J., 212, 234-242. [DOI], [ADS] (Cited on page 39.)

Low, B.C., 1996, "Solar Activity and the Corona", Solar Phys., 167, 217-265. [DOI], [ADS] (Cited on pages $10,32,40$, and 57.)

Low, B.C., 2001, "Coronal mass ejections, magnetic flux ropes, and solar magnetism", J. Geophys. Res., 106(A11), 25,141-25,163. [DOI], [ADS] (Cited on page 10.)

Low, B.C., 2006, "Spontaneous Current Sheets in an Ideal Hydromagnetic Fluid", Astrophys. J., 649, 1064-1077. [DOI], [ADS] (Cited on page 9.)

Low, B.C. and Hundhausen, J.R., 1995, "Magnetostatic structures of the solar corona. II. The magnetic topology of quiescent prominences", Astrophys. J., 443, 818-836. [DOI], [ADS] (Cited on pages 31, 32, and 57.)

Low, B.C. and Nakagawa, Y., 1975, "Dynamics of solar magnetic fields. VI", Astrophys. J., 199, 237-246. [DOI], [ADS] (Cited on page 39.)

Lu, E.T. and Hamilton, R.J., 1991, "Avalanches and the distribution of solar flares", Astrophys. J. Lett., 380, L89-L92. [DOI], [ADS] (Cited on page 21.)

Lynden-Bell, D. and Boily, C., 1994, "Self-Similar Solutions up to Flashpoint in Highly Wound Magnetostatics", Mon. Not. R. Astron. Soc., 267, 146. [ADS] (Cited on page 40.)

MacCombie, W.J. and Rust, D.M., 1979, "Physical parameters in long-decay coronal enhancements", Solar Phys., 61, 69-88. [DOI], [ADS] (Cited on page 12.)

Mackay, D.H. and van Ballegooijen, A.A., 2005, "New Results in Modeling the Hemispheric Pattern of Solar Filaments", Astrophys. J. Lett., 621, L77-L80. [DOI], [ADS] (Cited on page 32.) 
MacNeice, P., Burgess, A., McWhirter, R.W.P. and Spicer, D.S., 1984, "A numerical model of a solar flare based on electron beam heating of the chromosphere", Solar Phys., 90, 357-382. [DOI], [ADS] (Cited on page 69.)

MacTaggart, D. and Hood, A.W., 2009, "On the emergence of toroidal flux tubes: general dynamics and comparisons with the cylinder model", Astron. Astrophys., 507, 995-1004. [DOI], [ADS], [arXiv:0909.3987 [astro-ph.SR]] (Cited on page 30.)

Magara, T., 2001, "Dynamics of Emerging Flux Tubes in the Sun", Astrophys. J., 549, 608-628. [DOI], [ADS] (Cited on pages 25, 27, and 30.)

Magara, T., 2004, "A Model for Dynamic Evolution of Emerging Magnetic Fields in the Sun", Astrophys. J., 605, 480-492. [DOI], [ADS] (Cited on pages 29, 34, 35, and 36.)

Magara, T., 2006, "Dynamic and Topological Features of Photospheric and Coronal Activities Produced by Flux Emergence in the Sun", Astrophys. J., 653, 1499-1509. [DOI], [ADS] (Cited on page 35.)

Magara, T., 2007, "A Possible Structure of the Magnetic Field in Solar Filaments Obtained by Flux Emergence", Publ. Astron. Soc. Japan, 59, L51-L55. [ADS] (Cited on pages 32 and 33.)

Magara, T., 2008, "Emergence of a Partially Split Flux Tube into the Solar Atmosphere", Publ. Astron. Soc. Japan, 60, 809-826. [ADS] (Cited on pages 41 and 43.)

Magara, T., 2011, "A Possible Mechanism of Flux Cancellation via U-Loop Emergence on the Sun", Publ. Astron. Soc. Japan, 63, 417-426. [ADS] (Cited on pages 32, 36, 55, and 57.)

Magara, T. and Longcope, D.W., 2001, "Sigmoid Structure of an Emerging Flux Tube", Astrophys. J. Lett., 559, L55-L59. [DOI], [ADS] (Cited on pages 35, 36, and 55.)

Magara, T. and Longcope, D.W., 2003, "Injection of Magnetic Energy and Magnetic Helicity into the Solar Atmosphere by an Emerging Magnetic Flux Tube", Astrophys. J., 586, 630-649. [DOI], [ADS] (Cited on pages 28,29 , and 42 .)

Magara, T. and Shibata, K., 1999, "Evolution of Eruptive Flares. II. The Occurrence of Locally Enhanced Resistivity", Astrophys. J., 514, 456-471. [DOI], [ADS] (Cited on page 49.)

Magara, T. and Tsuneta, S., 2008, "Hinode's Observational Result on the Saturation of Magnetic Helicity Injected into the Solar Atmosphere and Its Relation to the Occurrence of a Solar Flare", Publ. Astron. Soc. Japan, 60, 1181-1189. [ADS] (Cited on page 44.)

Magara, T., Mineshige, S., Yokoyama, T. and Shibata, K., 1996, "Numerical Simulation of Magnetic Reconnection in Eruptive Flares", Astrophys. J., 466, 1054. [DOI], [ADS] (Cited on pages 63 and 69.)

Magara, T., Shibata, K. and Yokoyama, T., 1997, "Evolution of Eruptive Flares. I. Plasmoid Dynamics in Eruptive Flares", Astrophys. J., 487, 437. [DOI], [ADS] (Cited on pages 64, 65, and 69.)

Magara, T., Chen, P., Shibata, K. and Yokoyama, T., 2000, "A Unified Model of Coronal Mass Ejectionrelated Type II Radio Bursts", Astrophys. J. Lett., 538, L175-L178. [DOI], [ADS] (Cited on page 69.)

Manchester IV, W.B., 2001, "The Role of Nonlinear Alfvén Waves in Shear Formation during Solar Magnetic Flux Emergence", Astrophys. J., 547, 503-519. [DOI], [ADS] (Cited on pages 30, 55, and 56.)

Manchester IV, W.B., 2007, "Solar Atmospheric Dynamic Coupling Due to Shear Motions Driven by the Lorentz Force", Astrophys. J., 666, 532-540. [DOI], [ADS] (Cited on pages 30 and 55.)

Manchester IV, W.B., Gombosi, T., DeZeeuw, D. and Fan, Y., 2004, "Eruption of a Buoyantly Emerging Magnetic Flux Rope", Astrophys. J., 610, 588-596. [DOI], [ADS] (Cited on pages 30, 37, and 55.)

Manheimer, W.M. and Klein, H.H., 1975, "Model for the production and transport of energetic particles in a laser produced plasma", Phys. Fluids, 18, 1299-1307. [DOI], [ADS] (Cited on page 66.) 
Mariska, J.T., Emslie, A.G. and Li, P., 1989, "Numerical simulations of impulsively heated solar flares", Astrophys. J., 341, 1067-1074. [DOI], [ADS] (Cited on page 69.)

Martin, S.F., 1990, "Conditions for the Formation of Prominences as Inferred from Optical Observations", in IAU Colloq. 117: Dynamics of Quiescent Prominences, Proceedings of the No. 117 Colloquium of the IAU, Hvar, SR Croatia, Yugoslavia 1989, (Eds.) Ruždjak, V., Tandberg-Hanssen, E., vol. 363 of Lecture Notes in Physics, pp. 1-48, Springer, Berlin; New York. [DOI], [ADS] (Cited on page 30.)

Martin, S.F., 1998, "Conditions for the Formation and Maintenance of Filaments (Invited Review)", Solar Phys., 182, 107-137. [DOI], [ADS] (Cited on pages 30, 32, and 33.)

Martin, S.F., Dezso, L., Antalova, A., Kucera, A. and Harvey, K.L., 1982, "Emerging magnetic flux, flares and filaments - FBS interval 16-23 June 1980", Adv. Space Res., 2, 39-51. [DOI], [ADS] (Cited on page 9.)

Martin, S.F., Bilimoria, R. and Tracadas, P.W., 1994, "Magnetic field configurations basic to filament channels and filaments", in Solar Surface Magnetism, Proceedings of the NATO Advanced Research Workshop, held Soesterberg, the Netherlands, November 1-5, 1993, (Eds.) Rutten, R.J., Schrijver, C.J., NATO Science Series C, p. 303, Kluwer Academics, Dordrecht. [ADS] (Cited on page 30.)

Martres, M.J., Michard, R., Soru-Iscovici, I. and Tsap, T., 1968, "A Study of the Localization of Flares in Selected Active Regions", in Structure and Development of Solar Active Regions, Budapest, Hungary, 4-8 September 1967, (Ed.) Kiepenheuer, K.O., vol. 35 of IAU Symposia, p. 318, Reidel, Dordrecht. [ADS] (Cited on page 9.)

Masson, S., Pariat, E., Aulanier, G. and Schrijver, C.J., 2009, "The Nature of Flare Ribbons in Coronal Null-Point Topology", Astrophys. J., 700, 559-578. [DOI], [ADS] (Cited on page 55.)

Masuda, S., 1994, Hard X-ray Sources and the Primary Energy Release Site in Solar Flares: Finding of Loop-Top Impulsive Sources, Ph.D. Thesis, University of Tokyo, Tokyo. [ADS] (Cited on pages 16, 21, 63, and 68.)

Masuda, S., Kosugi, T., Hara, H., Tsuneta, S. and Ogawara, Y., 1994, "A loop-top hard X-ray source in a compact solar flare as evidence for magnetic reconnection", Nature, 371, 495-497. [DOI], [ADS] (Cited on pages 15,47 , and 61 .)

Masuda, S., Kosugi, T., Tsuneta, S. and Hara, H., 1996, "Discovery of a loop-top hard X-ray source in impulsive solar flares", Adv. Space Res., 17, 63. [DOI], [ADS] (Cited on page 15.)

Matsumoto, R., Tajima, T., Chou, W., Okubo, A. and Shibata, K., 1998, "Formation of a Kinked Alignment of Solar Active Regions", Astrophys. J. Lett., 493, L43. [DOI], [ADS] (Cited on page 35.)

McAllister, A., Uchida, Y., Tsuneta, S., Strong, K.T., Acton, L.W., Hiei, E., Bruner, M.E., Watanabe, T. and Shibata, K., 1992, "The Structure of the Coronal Soft X-Ray Source Associated with the Dark Filament Disappearance of 1991 September 28 Using the Yohkoh Soft X-Ray Telescope", Publ. Astron. Soc. Japan, 44, L205-L210. [ADS] (Cited on page 14.)

McAllister, A.H., Dryer, M., McIntosh, P., Singer, H. and Weiss, L., 1996, "A large polar crown coronal mass ejection and a 'problem' geomagnetic storm: April 14-23, 1994", J. Geophys. Res., 101(A6), 13,497-13,516. [DOI], [ADS] (Cited on page 14.)

McKenzie, D.E., 2000, "Supra-arcade Downflows in Long-Duration Solar Flare Events", Solar Phys., 195, 381-399. [ADS] (Cited on page 21.)

McKenzie, D.E. and Canfield, R.C., 2008, "Hinode XRT observations of a long-lasting coronal sigmoid", Astron. Astrophys., 481, L65-L68. [DOI], [ADS] (Cited on page 32.)

McKenzie, D.E. and Hudson, H.S., 1999, "X-Ray Observations of Motions and Structure above a Solar Flare Arcade", Astrophys. J. Lett., 519, L93-L96. [DOI], [ADS] (Cited on page 21.) 
Melrose, D.B., 1980, Plasma Astrophysics: Nonthermal Processes in Diffuse Magnetized Plasmas, Gordon and Breach, New York; London (Cited on page 10.)

Melrose, D.B., 1997, "A Solar Flare Model Based on Magnetic Reconnection between Current-carrying Loops", Astrophys. J., 486, 521. [DOI], [ADS] (Cited on pages 8 and 21.)

Mikić, Z. and Lee, M.A., 2006, "An Introduction to Theory and Models of CMEs, Shocks, and Solar Energetic Particles", Space Sci. Rev., 123, 57-80. [DOI], [ADS] (Cited on page 10.)

Mikić, Z. and Linker, J.A., 1994, "Disruption of coronal magnetic field arcades", Astrophys. J., 430, 898-912. [DOI], [ADS] (Cited on page 40.)

Mikić, Z., Barnes, D.C. and Schnack, D.D., 1988, "Dynamical evolution of a solar coronal magnetic field arcade", Astrophys. J., 328, 830-847. [DOI], [ADS] (Cited on pages 55 and 56.)

Miklenic, C.H., Veronig, A.M. and Vršnak, B., 2009, "Temporal comparison of nonthermal flare emission and magnetic-flux change rates", Astron. Astrophys., 499, 893-904. [DOI], [ADS], [arXiv:0910.1701 [astro-ph.SR]] (Cited on page 46.)

Miller, J.A., 1997, "Electron Acceleration in Solar Flares by Fast Mode Waves: Quasi-linear Theory and Pitch-Angle Scattering", Astrophys. J., 491, 939. [DOI], [ADS] (Cited on page 10.)

Miller, J.A., Cargill, P.J., Emslie, A.G., Holman, G.D., Dennis, B.R., LaRosa, T.N., Winglee, R.M., Benka, S.G. and Tsuneta, S., 1997, "Critical issues for understanding particle acceleration in impulsive solar flares", J. Geophys. Res., 102, 14,631-14,660. [DOI], [ADS] (Cited on page 70.)

Miyagoshi, T. and Yokoyama, T., 2004, "Magnetohydrodynamic Simulation of Solar Coronal Chromospheric Evaporation Jets Caused by Magnetic Reconnection Associated with Magnetic Flux Emergence", Astrophys. J., 614, 1042-1053. [DOI], [ADS] (Cited on page 25.)

Moon, Y.-J., Chae, J., Wang, H., Choe, G.S. and Park, Y.D., 2002, "Impulsive Variations of the Magnetic Helicity Change Rate Associated with Eruptive Flares", Astrophys. J., 580, 528-537. [DOI], [ADS] (Cited on page 44.)

Moore, R.L., Sterling, A.C., Hudson, H.S. and Lemen, J.R., 2001, "Onset of the Magnetic Explosion in Solar Flares and Coronal Mass Ejections", Astrophys. J., 552, 833-848. [DOI], [ADS] (Cited on pages 57 and 58.)

Moreno-Insertis, F., Galsgaard, K. and Ugarte-Urra, I., 2008, "Jets in Coronal Holes: Hinode Observations and Three-dimensional Computer Modeling", Astrophys. J. Lett., 673, L211-L214. [DOI], [ADS], [arXiv:0712.1059] (Cited on pages 52 and 55.)

Moreton, G.E., 1960, "H $\alpha$ Observations of Flare-Initiated Disturbances with Velocities 1000 km/sec", Astron. J., 65, 494-495. [DOI], [ADS] (Cited on page 69.)

Moreton, G.E. and Ramsey, H.E., 1960, "Recent Observations of Dynamical Phenomena Associated with Solar Flares", Publ. Astron. Soc. Pac., 72, 357. [DOI], [ADS] (Cited on page 69.)

Moses, D., Clette, F., Delaboudinière, J.-P., Artzner, G.E., Bougnet, M., Brunaud, J., Carabetian, C., Gabriel, A.H., Hochedez, J.F., Millier, F., Song, X.Y., Au, B., Dere, K.P., Howard, R.A., Kreplin, R., Michels, D.J., Defise, J.M., Jamar, C., Rochus, P., Chauvineau, J.P., Marioge, J.P., Catura, R.C., Lemen, J.R., Shing, L., Stern, R.A., Gurman, J.B., Neupert, W.M., Newmark, J., Thompson, B., Maucherat, A., Portier-Fozzani, F., Berghmans, D., Cugnon, P., van Dessel, E.L. and Gabryl, J.R., 1997, "EIT Observations of the Extreme Ultraviolet Sun", Solar Phys., 175, 571-599. [DOI], [ADS] (Cited on page 70.)

Mouschovias, T.C. and Poland, A.I., 1978, "Expansion and broadening of coronal loop transients: A theoretical explanation", Astrophys. J., 220,675-682. [DOI], [ADS] (Cited on page 57.) 
Murray, M.J. and Hood, A.W., 2007, "Simple emergence structures from complex magnetic fields", Astron. Astrophys., 470, 709-719. [DOI], [ADS] (Cited on page 30.)

Murray, M.J. and Hood, A.W., 2008, "Emerging flux tubes from the solar interior into the atmosphere: effects of non-constant twist", Astron. Astrophys., 479, 567-577. [DOI], [ADS] (Cited on pages 28 and 30.)

Murray, M.J., Hood, A.W., Moreno-Insertis, F., Galsgaard, K. and Archontis, V., 2006, "3D simulations identifying the effects of varying the twist and field strength of an emerging flux tube", Astron. Astrophys., 460, 909-923. [DOI], [ADS] (Cited on page 30.)

Nagai, F., 1980, "A model of hot loops associated with solar flares. I. Gasdynamics in the loops", Solar Phys., 68, 351-379. [DOI], [ADS] (Cited on page 69.)

Nagai, F. and Emslie, A.G., 1984, "Gas dynamics in the impulsive phase of solar flares. I. Thick-target heating by nonthermal electrons", Astrophys. J., 279, 896-908. [DOI], [ADS] (Cited on page 69.)

Nagashima, K. and Yokoyama, T., 2006, "Statistical Study of the Reconnection Rate in Solar Flares Observed with Yohkoh SXT", Astrophys. J., 647, 654-661. [DOI], [ADS], [arXiv:astro-ph/0605712] (Cited on page 46.)

Nakagawa, Y., Raadu, M.A., Billings, D.E. and McNamara, D., 1971, "On the Topology of Filaments and Chromospheric Fibrils near Sunspots", Solar Phys., 19, 72-85. [DOI], [ADS] (Cited on page 39.)

Narukage, N. and Shibata, K., 2006, "Statistical Analysis of Reconnection Inflows in Solar Flares Observed with SOHO EIT", Astrophys. J., 637, 1122-1134. [DOI], [ADS] (Cited on pages 21 and 46.)

Narukage, N., Hudson, H.S., Morimoto, T., Akiyama, S., Kitai, R., Kurokawa, H. and Shibata, K., 2002, "Simultaneous Observation of a Moreton Wave on 1997 November 3 in H $\alpha$ and Soft X-Rays", Astrophys. J. Lett., 572, L109-L112. [DOI], [ADS] (Cited on page 70.)

Neupert, W.M., 1968, "Comparison of Solar X-Ray Line Emission with Microwave Emission during Flares", Astrophys. J. Lett., 153, L59. [DOI], [ADS] (Cited on page 61.)

Newcomb, W.A., 1961, "Convective Instability Induced by Gravity in a Plasma with a Frozen-In Magnetic Field", Phys. Fluids, 4, 391-396. [DOI], [ADS] (Cited on page 28.)

Nindos, A. and Zhang, H., 2002, "Photospheric Motions and Coronal Mass Ejection Productivity", Astrophys. J. Lett., 573, L133-L136. [DOI], [ADS] (Cited on page 44.)

Nishida, K., Shimizu, M., Shiota, D., Takasaki, H., Magara, T. and Shibata, K., 2009, "Numerical Examination of Plasmoid-Induced Reconnection Model for Solar Flares: The Relation between Plasmoid Velocity and Reconnection Rate", Astrophys. J., 690, 748-757. [DOI], [ADS], [arXiv:0809.0797] (Cited on page 49.)

Nishizuka, N., Asai, A., Takasaki, H., Kurokawa, H. and Shibata, K., 2009, "The Power-Law Distribution of Flare Kernels and Fractal Current Sheets in a Solar Flare", Astrophys. J. Lett., 694, L74-L78. [DOI], [ADS] (Cited on page 49.)

Nitta, N. and Yaji, K., 1997, "A Superhot Flare Observed by Yohkoh", Astrophys. J., 484, 927-936. [DOI], [ADS] (Cited on page 68.)

Nitta, S., 2010, "Observational Prediction of High Magnetic Reynolds Number Pre-flare Reconnection Events: An Application of Nitta's Self-similar Reconnection Model", Astrophys. J., 719, 1828-1843. [DOI], [ADS] (Cited on page 47.)

Nozawa, S., 2005, "Three-Dimensional Magnetohydrodynamic Simulation of Nonlinear Magnetic Buoyancy Instability of Flux Sheets with Magnetic Shear", Publ. Astron. Soc. Japan, 57, 995-1007. [ADS] (Cited on page 30 .) 
Nozawa, S., Shibata, K., Matsumoto, R., Sterling, A.C., Tajima, T., Uchida, Y., Ferrari, A. and Rosner, R., 1992, "Emergence of magnetic flux from the convection zone into the solar atmosphere. I. Linear and nonlinear adiabatic evolution of the convective-Parker instability", Astrophys. J. Suppl. Ser., 78, 267-282. [DOI], [ADS] (Cited on page 25.)

Ohyama, M. and Shibata, K., 1997, "Preflare Heating and Mass Motion in a Solar Flare Associated with Hot Plasma Ejection: 1993 November 11 C9.7 Flare", Publ. Astron. Soc. Japan, 49, 249-261. [ADS] (Cited on pages 21, 64, and 65.)

Ohyama, M. and Shibata, K., 1998, "X-Ray Plasma Ejection Associated with an Impulsive Flare on 1992 October 5: Physical Conditions of X-Ray Plasma Ejection", Astrophys. J., 499, 934. [DOI], [ADS] (Cited on pages 13 and 21.)

Okamoto, T.J., Nakai, H., Keiyama, A., Narukage, N., UeNo, S., Kitai, R., Kurokawa, H. and Shibata, K., 2004, "Filament Oscillations and Moreton Waves Associated with EIT Waves", Astrophys. J., 608, 1124-1132. [DOI], [ADS] (Cited on page 70.)

Okamoto, T.J., Tsuneta, S., Berger, T.E., Ichimoto, K., Katsukawa, Y., Lites, B.W., Nagata, S., Shibata, K., Shimizu, T., Shine, R.A., Suematsu, Y., Tarbell, T.D. and Title, A.M., 2007, "Coronal Transverse Magnetohydrodynamic Waves in a Solar Prominence", Science, 318, 1577. [DOI], [ADS], [arXiv:0801.1958] (Cited on page 32.)

Okamoto, T.J., Tsuneta, S., Lites, B.W., Kubo, M., Yokoyama, T., Berger, T.E., Ichimoto, K., Katsukawa, Y., Nagata, S., Shibata, K., Shimizu, T., Shine, R.A., Suematsu, Y., Tarbell, T.D. and Title, A.M., 2008, "Emergence of a Helical Flux Rope under an Active Region Prominence", Astrophys. J. Lett., 673, L215-L218. [DOI], [ADS], [arXiv:0801.1956] (Cited on page 32.)

Otsuji, K., Shibata, K., Kitai, R., Ueno, S., Nagata, S., Matsumoto, T., Nakamura, T., Watanabe, H., Tsuneta, S., Suematsu, Y., Ichimoto, K., Shimizu, T., Katsukawa, Y., Tarbell, T.D., Lites, B., Shine, R.A. and Title, A.M., 2007, "Small-Scale Magnetic-Flux Emergence Observed with Hinode Solar Optical Telescope", Publ. Astron. Soc. Japan, 59, 649. [ADS], [arXiv:0709.3207] (Cited on page 23.)

Pallavicini, R., Serio, S. and Vaiana, G.S., 1977, "A survey of soft X-ray limb flare images: The relation between their structure in the corona and other physical parameters", Astrophys. J., 216, 108-122. [DOI], [ADS] (Cited on page 15.)

Pariat, E., Aulanier, G., Schmieder, B., Georgoulis, M.K., Rust, D.M. and Bernasconi, P.N., 2004, "Resistive Emergence of Undulatory Flux Tubes", Astrophys. J., 614, 1099-1112. [DOI], [ADS] (Cited on pages 27 and 28.)

Pariat, E., Démoulin, P. and Berger, M.A., 2005, "Photospheric flux density of magnetic helicity", Astron. Astrophys., 439, 1191-1203. [DOI], [ADS] (Cited on page 44.)

Park, S.-H., Chae, J., Jing, J., Tan, C. and Wang, H., 2010, "Time Evolution of Coronal Magnetic Helicity in the Flaring Active Region NOAA 10930", Astrophys. J., 720, 1102-1107. [DOI], [ADS], [arXiv:1008.1558 [astro-ph.SR]] (Cited on page 41.)

Parker, E.N., 1955, "The Formation of Sunspots from the Solar Toroidal Field", Astrophys. J., 121, 491-507. [DOI], [ADS] (Cited on page 25.)

Parker, E.N., 1957, "Sweet's Mechanism for Merging Magnetic Fields in Conducting Fluids", J. Geophys. Res., 62(4), 509-520. [DOI], [ADS] (Cited on pages 7 and 46.)

Parker, E.N., 1963, "The Solar-Flare Phenomenon and the Theory of Reconnection and Annihiliation of Magnetic Fields", Astrophys. J. Suppl. Ser., 8, 177-211. [DOI], [ADS] (Cited on pages 7 and 46.)

Parker, E.N., 1978, "Hydraulic concentration of magnetic fields in the solar photosphere. VI. Adiabatic cooling and concentration in downdrafts", Astrophys. J., 221, 368-377. [DOI], [ADS] (Cited on page 25.) 
Parker, E.N., 1979, Cosmical Magnetic Fields: Their Origin and their Activity, International Series of Monographs on Physics, Clarendon Press; Oxford University Press, Oxford; New York. [ADS] (Cited on pages 8 and 10.)

Parker, E.N., 1994, Spontaneous Current Sheets in Magnetic Fields: With Applications to Stellar X-Rays, vol. 2 of International Series on Astronomy and Astrophysics, Oxford University Press, Oxford; New York. [ADS], [Google Books] (Cited on pages 9 and 10.)

Peres, G., Reale, F., Serio, S. and Pallavicini, R., 1987, "Hydrodynamic flare modeling: Comparison of numerical calculations with SMM observations of the 1980 November 12 17:00 UT flare", Astrophys. J., 312, 895-908. [DOI], [ADS] (Cited on page 69.)

Petschek, H.E., 1964, "Magnetic Field Annihilation", in The Physics of Solar Flares, Proceedings of the AAS-NASA Symposium held 28-30 October, 1963 at the Goddard Space Flight Center, Greenbelt, MD, (Ed.) Hess, W.N., vol. SP-50 of NASA Special Publications, pp. 425-439, NASA Science and Technical Information Division, Washington, DC. [ADS] (Cited on pages 7, 8, and 46.)

Pevtsov, A.A., 2002, "Active-Region Filaments and X-ray Sigmoids", Solar Phys., 207, 111-123. [ADS] (Cited on page 32.)

Pevtsov, A.A., Canfield, R.C. and Metcalf, T.R., 1995, "Latitudinal variation of helicity of photospheric magnetic fields", Astrophys. J. Lett., 440, L109-L112. [DOI], [ADS] (Cited on page 30.)

Pevtsov, A.A., Balasubramaniam, K.S. and Rogers, J.W., 2003, "Chirality of Chromospheric Filaments", Astrophys. J., 595, 500-505. [DOI], [ADS] (Cited on page 32.)

Priest, E.R. (Ed.), 1981, Solar Flare Magnetohydrodynamics, vol. 1 of The Fluid Mechanics of Astrophysics and Geophysics, Gordon and Breach, New York. [Google Books] (Cited on page 10.)

Priest, E.R., 1982, Solar Magnetohydrodynamics, vol. 21 of Geophysics and Astrophysics Monographs, Reidel, Dordrecht; Boston. [ADS], [Google Books] (Cited on page 10.)

Priest, E.R., 1985, "The magnetohydrodynamics of current sheets", Rep. Prog. Phys., 48, 955-1090. [DOI], [ADS] (Cited on page 49.)

Priest, E.R. and Démoulin, P., 1995, "Three-dimensional magnetic reconnection without null points, 1. Basic theory of magnetic flipping", J. Geophys. Res., 100, 23,443-23,464. [DOI], [ADS] (Cited on page 55.)

Priest, E.R. and Forbes, T., 2000, Magnetic Reconnection: MHD Theory and Applications, Cambridge University Press, Cambridge; New York. [ADS], [Google Books] (Cited on pages 10, 41, 45, and 46.)

Priest, E.R. and Forbes, T.G., 2002, "The magnetic nature of solar flares", Astron. Astrophys. Rev., 10, 313-377. [DOI], [ADS] (Cited on pages 8 and 10.)

Ramaty, R. and Murphy, R.J., 1987, "Nuclear processes and accelerated particles in solar flares", Space Sci. Rev., 45, 213-268. [DOI], [ADS] (Cited on pages 10 and 70.)

Raymond, J.C., Cox, D.P. and Smith, B.W., 1976, "Radiative cooling of a low-density plasma", Astrophys. J., 204, 290-292. [DOI], [ADS] (Cited on page 68.)

Régnier, S. and Amari, T., 2004, "3D magnetic configuration of the $\mathrm{H} \alpha$ filament and X-ray sigmoid in NOAA AR 8151", Astron. Astrophys., 425, 345-352. [DOI], [ADS] (Cited on page 32.)

Régnier, S. and Priest, E.R., 2007, "Free Magnetic Energy in Solar Active Regions above the MinimumEnergy Relaxed State", Astrophys. J. Lett., 669, L53-L56. [DOI], [ADS], [arXiv:0805.1619] (Cited on page 39.)

Rogers, B.N., Denton, R.E. and Drake, J.F., 2003, "Signatures of collisionless magnetic reconnection", J. Geophys. Res., 108(A3), 1111. [DOI], [ADS] (Cited on page 49.) 
Roussev, I.I., Forbes, T.G., Gombosi, T.I., Sokolov, I.V., DeZeeuw, D.L. and Birn, J., 2003, "A Threedimensional Flux Rope Model for Coronal Mass Ejections Based on a Loss of Equilibrium", Astrophys. J. Lett., 588, L45-L48. [DOI], [ADS] (Cited on page 57.)

Rust, D.M., 1968, "Chromospheric Explosions and Satellite Sunspots", in Structure and Development of Solar Active Regions, Symposium no. 35 held in Budapest, Hungary, 4-8 September 1967, (Ed.) Kiepenheuer, K.O., vol. 35 of IAU Symposium, p. 77, Reidel, Dordrecht. [ADS] (Cited on page 9.)

Rust, D.M., 1972, "Flares and Changing Magnetic Fields", Solar Phys., 25, 141-157. [DOI], [ADS] (Cited on page 50.)

Rust, D.M. and Kumar, A., 1994, "Helical magnetic fields in filaments", Solar Phys., 155, 69-97. [DOI], [ADS] (Cited on pages 30 and 32.)

Rust, D.M. and Kumar, A., 1996, "Evidence for Helically Kinked Magnetic Flux Ropes in Solar Eruptions", Astrophys. J. Lett., 464, L199-L202. [DOI], [ADS] (Cited on page 30.)

Sakai, J.-I. and de Jager, C., 1996, "Solar Flares and Collisions Between Current-Carrying Loops Types and Mechanisms of Solar Flares and Coronal Loop Heating", Space Sci. Rev., 77, 1-192. [DOI], [ADS] (Cited on page 10.)

Sakai, J.-I. and Koide, S., 1992, "Classification of magnetic reconnection during two current-loop coalescence", Solar Phys., 142, 399-402. [DOI], [ADS] (Cited on page 17.)

Sakai, J.-I. and Ohsawa, Y., 1987, "Particle acceleration by magnetic reconnection and shocks during current loop coalescence in solar flares", Space Sci. Rev., 46, 113-198. [ADS] (Cited on pages 10 and 49.)

Sakurai, T., 1979, "A New Approach to the Force-Free Field and Its Application to the Magnetic Field of Solar Active Regions", Publ. Astron. Soc. Japan, 31, 209-230. [ADS] (Cited on pages 8 and 39.)

Sato, T. and Hayashi, T., 1979, "Externally driven magnetic reconnection and a powerful magnetic energy converter", Phys. Fluids, 22, 1189-1202. [DOI], [ADS] (Cited on page 47.)

Schmieder, B., Aulanier, G., Mein, P. and López Ariste, A., 2006, "Evolving Photospheric Flux Concentrations and Filament Dynamic Changes", Solar Phys., 238, 245-259. [DOI], [ADS] (Cited on page 30.)

Scholer, M., 1989, "Undriven magnetic reconnection in an isolated current sheet", J. Geophys. Res., 94, 8805-8812. [DOI], [ADS] (Cited on page 47.)

Shay, M.A., Drake, J.F., Rogers, B.N. and Denton, R.E., 2001, "Alfvénic collisionless magnetic reconnection and the Hall term", J. Geophys. Res., 106, 3759-3772. [DOI], [ADS] (Cited on page 49.)

Sheeley Jr, N.R. and Harvey, J.W., 1975, "The calculation of force-free fields from discrete flux distributions", Solar Phys., 45, 275-290. [DOI], [ADS] (Cited on page 39.)

Shibata, K., 1991, "Theoretical models of solar flares", in Flare Physics in Solar Activity Maximum 22, Proceedings of the International SOLAR-A Science Meeting held at Tokyo, Japan, 23-26 October 1990, (Eds.) Uchida, Y., Canfield, R.C., Watanabe, T., Hiei, E., vol. 387 of Lecture Notes in Physics, pp. 205-218, Springer, Berlin; New York. [DOI] (Cited on page 8.)

Shibata, K., 1997, "Rapidly Time Variable Phenomena: Jets, Explosive Events, and Flares", in The Corona and Solar Wind Near Minimum Activity, Fifth SOHO Workshop, Oslo, Norway, 17 -20 June 1997, (Ed.) Wilson, A., vol. SP-404 of ESA Conference Proceedings, pp. 103-112, ESA, Noordwijk (Cited on page 10.)

Shibata, K., 1999, "Evidence of Magnetic Reconnection in Solar Flares and a Unified Model of Flares", Astrophys. Space Sci., 264, 129-144. [ADS] (Cited on pages 8, 10, 18, 20, and 49.) 
Shibata, K. and Tanuma, S., 2001, "Plasmoid-induced-reconnection and fractal reconnection", Earth Planets Space, 53, 473-482. [ADS], [arXiv:astro-ph/0101008] (Cited on pages 48, 49, and 64.)

Shibata, K. and Yokoyama, T., 1999, "Origin of the Universal Correlation between the Flare Temperature and the Emission Measure for Solar and Stellar Flares", Astrophys. J. Lett., 526, L49-L52. [DOI], [ADS] (Cited on pages 69, 71, and 72.)

Shibata, K., Tajima, T., Matsumoto, R., Horiuchi, T., Hanawa, T., Rosner, R. and Uchida, Y., 1989a, "Nonlinear Parker instability of isolated magnetic flux in a plasma", Astrophys. J., 338, 471-492. [DOI], [ADS] (Cited on page 25.)

Shibata, K., Tajima, T., Steinolfson, R.S. and Matsumoto, R., 1989b, "Two-dimensional magnetohydrodynamic model of emerging magnetic flux in the solar atmosphere", Astrophys. J., 345, 584-596. [DOI], [ADS] (Cited on pages 25, 26, and 50.)

Shibata, K., Nozawa, S., Matsumoto, R., Sterling, A.C. and Tajima, T., 1990a, "Emergence of solar magnetic flux from the convection zone into the photosphere and chromosphere", Astrophys. J. Lett., 351, L25-L28. [DOI], [ADS] (Cited on page 25.)

Shibata, K., Tajima, T. and Matsumoto, R., 1990b, "Self-similar evolution of the nonlinear magnetic buoyancy instability", Phys. Fluids B, 2, 1989-2000. [DOI], [ADS] (Cited on page 25.)

Shibata, K., Ishido, Y., Acton, L.W., Strong, K.T., Hirayama, T., Uchida, Y., McAllister, A.H., Matsumoto, R., Tsuneta, S., Shimizu, T., Hara, H., Sakurai, T., Ichimoto, K., Nishino, Y. and Ogawara, Y., 1992a, "Observations of X-Ray Jets with the Yohkoh Soft X-Ray Telescope", Publ. Astron. Soc. Japan, 44, L173-L179. [ADS] (Cited on pages 17 and 18.)

Shibata, K., Nozawa, S. and Matsumoto, R., 1992b, "Magnetic reconnection associated with emerging magnetic flux", Publ. Astron. Soc. Japan, 44, 265-272. [ADS] (Cited on page 50.)

Shibata, K., Masuda, S., Shimojo, M., Hara, H., Yokoyama, T., Tsuneta, S., Kosugi, T. and Ogawara, Y., 1995, "Hot-Plasma Ejections Associated with Compact-Loop Solar Flares", Astrophys. J. Lett., 451, L83. [DOI], [ADS] (Cited on pages 9, 16, 20, and 21.)

Shimizu, M., Nishida, K., Takasaki, H., Shiota, D., Magara, T. and Shibata, K., 2008, "The Correlation among the Rise Velocity of a Soft X-Ray Loop, the Ejection Velocity of a Plasmoid, and the Height above the Loop Top of the Hard X-Ray Source in Masuda-Type Flares, and Its Interpretation Based on the Reconnection Model of Flares", Astrophys. J. Lett., 683, L203-L206. [DOI], [ADS] (Cited on page 16.)

Shimizu, T., 1995, "Energetics and Occurrence Rate of Active-Region Transient Brightenings and Implications for the Heating of the Active-Region Corona", Publ. Astron. Soc. Japan, 47, 251-263. [ADS] (Cited on pages 17 and 20.)

Shimizu, T., Tsuneta, S., Acton, L.W., Lemen, J.R. and Uchida, Y., 1992, "Transient Brightenings in Active Regions Observed by the Soft X-Ray Telescope on Yohkoh", Publ. Astron. Soc. Japan, 44, L147-L153. [ADS] (Cited on page 17.)

Shimizu, T., Tsuneta, S., Acton, L.W., Lemen, J.R., Ogawara, Y. and Uchida, Y., 1994, "Morphology of active region transient brightenings with the YOHKOH Soft X-ray Telescope", Astrophys. J., 422, 906-911. [DOI], [ADS] (Cited on page 17.)

Shimojo, M., Hashimoto, S., Shibata, K., Hirayama, T., Hudson, H.S. and Acton, L.W., 1996, "Statistical Study of Solar X-Ray Jets Observed with the Yohkoh Soft X-Ray Telescope", Publ. Astron. Soc. Japan, 48, 123-136. [ADS] (Cited on page 17.)

Shimojo, M., Narukage, N., Kano, R., Sakao, T., Tsuneta, S., Shibasaki, K., Cirtain, J.W., Lundquist, L.L., Reeves, K.K. and Savcheva, A., 2007, "Fine Structures of Solar X-Ray Jets Observed with the X-Ray Telescope aboard Hinode", Publ. Astron. Soc. Japan, 59, S745-S750. [ADS] (Cited on page 20.) 
Shiota, D., Isobe, H., Chen, P.F., Yamamoto, T.T., Sakajiri, T. and Shibata, K., 2005, "Self-Consistent Magnetohydrodynamic Modeling of a Coronal Mass Ejection, Coronal Dimming, and a Giant Cuspshaped Arcade Formation", Astrophys. J., 634, 663-678. [DOI], [ADS], [arXiv:astro-ph/0508478] (Cited on pages 14 and 15.)

Somov, B.V., 2000, Cosmic Plasma Physics, vol. 251 of Astrophysics and Space Science Library, Kluwer Academic Publishers, Dordrecht; Boston (Cited on page 10.)

Somov, B.V. and Kosugi, T., 1997, "Collisionless Reconnection and High-Energy Particle Acceleration in Solar Flares", Astrophys. J., 485, 859. [DOI], [ADS] (Cited on page 61.)

Somov, B.V. and Titov, V.S., 1985, "Magnetic reconnection in a high-temperature plasma of solar flares. II. Effects caused by transverse and longitudinal magnetic fields", Solar Phys., 102, 79-96. [DOI], [ADS] (Cited on page 66.)

Somov, B.V., Spektor, A.R. and Syrovatskii, S.I., 1981, "Hydrodynamic response of the solar chromosphere to an elementary flare burst. I: Heating by accelerated electrons", Solar Phys., 73, 145-155. [DOI], [ADS] (Cited on page 69.)

Spicer, D.S., 1977, "An unstable arch model of a solar flare", Solar Phys., 53, 305-345. [DOI], [ADS] (Cited on page 15.)

Spitzer, L., 1962, Physics of Fully Ionized Gases, vol. 3 of Interscience Tracts on Physics and Astronomy, Interscience, New York, 2nd rev. edn. [ADS] (Cited on pages 7, 49, and 66.)

Spruit, H.C. and Zweibel, E.G., 1979, "Convective instability of thin flux tubes", Solar Phys., 62, 15-22. [DOI], [ADS] (Cited on page 25.)

Steinolfson, R.S. and van Hoven, G., 1984, "Nonlinear evolution of the resistive tearing mode", Phys. Fluids, 27, 1207-1214. [DOI], [ADS] (Cited on page 49.)

Sterling, A.C. and Hudson, H.S., 1997, "Yohkoh SXT Observations of X-Ray 'Dimming' Associated with a Halo Coronal Mass Ejection", Astrophys. J. Lett., 491, L55. [DOI], [ADS] (Cited on pages 15 and 32.)

Stix, M., 2004, The Sun: An Introduction, Astronomy and Astrophysics Library, Springer, Berlin; New York, 2nd corr. edn. [ADS], [Google Books] (Cited on page 10.)

Strous, L.H., Scharmer, G., Tarbell, T.D., Title, A.M. and Zwaan, C., 1996, "Phenomena in an emerging active region. I. Horizontal dynamics", Astron. Astrophys., 306, 947-959. [ADS] (Cited on page 23.)

Sturrock, P.A., 1966, "Model of the High-Energy Phase of Solar Flares", Nature, 211, 695-697. [DOI], [ADS] (Cited on pages 7 and 8.)

Sturrock, P.A., 1991, "Maximum energy of semi-infinite magnetic field configurations", Astrophys. J., 380, 655-659. [DOI], [ADS] (Cited on pages 39 and 40.)

Sturrock, P.A., 1992, "The Emerging Picture of Eruptive Solar Flares", in Eruptive Solar Flares, Proceedings of Colloquium No. 133 of the International Astronomical Union, held at Iguazú, Argentina, 2-6 August 1991, (Eds.) Švestka, Z., Jackson, B.V., Machado, M.E., vol. 399 of Lecture Notes in Physics, pp. 397-409, Springer, Berlin; New York. [DOI], [ADS] (Cited on page 8.)

Sturrock, P.A., Kaufman, P., Moore, R.L. and Smith, D.F., 1984, "Energy release in solar flares", Solar Phys., 94, 341-357. [DOI], [ADS] (Cited on page 57.)

Sturrock, P.A., Weber, M., Wheatland, M.S. and Wolfson, R., 2001, "Metastable Magnetic Configurations and Their Significance for Solar Eruptive Events", Astrophys. J., 548, 492-496. [DOI], [ADS] (Cited on page 57.)

Sui, L. and Holman, G.D., 2003, "Evidence for the Formation of a Large-Scale Current Sheet in a Solar Flare", Astrophys. J. Lett., 596, L251-L254. [DOI], [ADS] (Cited on page 21.) 
Sweet, P.A., 1958, "The neutral point theory of solar flares", in Electromagnetic Phenomena in Cosmical Physics, Proceedings of IAU Symposium No. 6, held in Stockholm, August 27-31, 1956, (Ed.) Lehnert, B., vol. 6 of IAU Symposium, pp. 123-134, Cambridge University Press, Cambridge; New York. [ADS] (Cited on pages 7 and 46.)

Tajima, T. and Shibata, K., 1997, Plasma Astrophysics, vol. 98 of Frontiers in Physics, Addison-Wesley, Reading, MA. [ADS] (Cited on pages 10, 25, and 48.)

Tajima, T., Sakai, J., Nakajima, H., Kosugi, T., Brunel, F. and Kundu, M.R., 1987, "Current loop coalescence model of solar flares", Astrophys. J., 321, 1031-1048. [DOI], [ADS] (Cited on pages 17 and 49.)

Tanaka, K., 1991, "Studies on a very flare-active $\delta$ group: Peculiar $\delta$ spot evolution and inferred subsurface magnetic rope structure", Solar Phys., 136, 133-149. [DOI], [ADS] (Cited on page 23.)

Tandberg-Hanssen, E. and Emslie, A.G., 1988, The Physics of Solar Flares, Cambridge Astrophysics Series, Cambridge University Press, Cambridge; New York. [Google Books] (Cited on page 10.)

TanDokoro, R. and Fujimoto, M., 2005, "Three-dimensional MHD simulation study of the structure at the leading part of a reconnection jet", Geophys. Res. Lett., 32, L23102. [DOI], [ADS] (Cited on page 21.)

Tanuma, S., Yokoyama, T., Kudoh, T. and Shibata, K., 2001, "Two-dimensional Magnetohydrodynamic Numerical Simulations of Magnetic Reconnection Triggered by a Supernova Shock in the Interstellar Medium: Generation of X-Ray Gas in the Galaxy", Astrophys. J., 551, 312-332. [DOI], [ADS], [arXiv:astro-ph/0009088] (Cited on page 49.)

Tarbell, T., Ferguson, S., Frank, Z., Title, A.M. and Topka, K.P., 1988, "High-Resolution Digital Movies of Emerging Flux and Horizontal Flows in Active Regions on the Sun", Bull. Am. Astron. Soc., 20, 680. [ADS] (Cited on page 23.)

Thompson, B.J., Plunkett, S.P., Gurman, J.B., Newmark, J.S., St Cyr, O.C. and Michels, D.J., 1998, "SOHO/EIT observations of an Earth-directed coronal mass ejection on May 12, 1997", Geophys. Res. Lett., 25, 2465-2468. [DOI], [ADS] (Cited on page 70.)

Titov, V.S. and Démoulin, P., 1999, "Basic topology of twisted magnetic configurations in solar flares", Astron. Astrophys., 351, 707-720. [ADS] (Cited on page 35.)

Török, T. and Kliem, B., 2005, "Confined and Ejective Eruptions of Kink-unstable Flux Ropes", Astrophys. J. Lett., 630, L97-L100. [DOI], [ADS], [arXiv:astro-ph/0507662] (Cited on page 57.)

Treumann, R.A. and Baumjohann, W., 1997, Advanced Space Plasma Physics, Imperial College Press, London. [ADS] (Cited on page 49.)

Tsuboi, Y., Koyama, K., Murakami, H., Hayashi, M., Skinner, S. and Ueno, S., 1998, "ASCA Detection of a Superhot 100 Million K X-Ray Flare on the Weak-lined T Tauri Star V773 Tauri", Astrophys. J., 503, 894-901. [DOI], [ADS] (Cited on page 71.)

Tsuneta, S., 1996, "Structure and Dynamics of Magnetic Reconnection in a Solar Flare", Astrophys. J., 456, 840. [DOI], [ADS] (Cited on pages 12, 15, 21, and 68.)

Tsuneta, S., 1997, "Moving Plasmoid and Formation of the Neutral Sheet in a Solar Flare", Astrophys. J., 483, 507. [DOI], [ADS] (Cited on pages 12 and 21.)

Tsuneta, S. and Naito, T., 1998, "Fermi Acceleration at the Fast Shock in a Solar Flare and the Impulsive Loop-Top Hard X-Ray Source", Astrophys. J. Lett., 495, L67-L71. [DOI], [ADS], [arXiv:astroph/9801109] (Cited on page 70.)

Tsuneta, S., Hara, H., Shimizu, T., Acton, L.W., Strong, K.T., Hudson, H.S. and Ogawara, Y., 1992a, "Observation of a solar flare at the limb with the YOHKOH Soft X-ray Telescope", Publ. Astron. Soc. Japan, 44, L63-L69. [ADS] (Cited on pages 12, 21, 32, 63, and 66.) 
Tsuneta, S., Takahashi, T., Acton, L.W., Bruner, M.E., Harvey, K.L. and Ogawara, Y., 1992b, "Global restructuring of the coronal magnetic fields observed with the YOHKOH Soft X-ray Telescope", Publ. Astron. Soc. Japan, 44, L211-L214. [ADS] (Cited on page 14.)

Uchida, Y., 1968, "Propagation of Hydromagnetic Disturbances in the Solar Corona and Moreton's Wave Phenomenon", Solar Phys., 4, 30-44. [DOI], [ADS] (Cited on page 69.)

Uchida, Y., 1974, "Behavior of the flare produced coronal MHD wavefront and the occurrence of type II radio bursts", Solar Phys., 39, 431-449. [DOI], [ADS] (Cited on page 69.)

Uchida, Y. and Shibata, K., 1988, "A magnetodynamic mechanism for the heating of emerging magnetic flux tubes and loop flares", Solar Phys., 116, 291-307. [DOI], [ADS] (Cited on pages 8, 15, and 21.)

Ugai, M., 1987, "Strong loop heating by the fast reconnection in a closed system", Geophys. Res. Lett., 14, 103-106. [DOI], [ADS] (Cited on page 69.)

Ugai, M. and Tsuda, T., 1977, "Magnetic field-line reconnexion by localized enhancement of resistivity. Part 1: Evolution in a compressible MHD fluid", J. Plasma Phys., 17, 337-356. [DOI], [ADS] (Cited on page 47.)

Uzdensky, D.A. and Kulsrud, R.M., 2000, "Two-dimensional numerical simulation of the resistive reconnection layer", Phys. Plasmas, 7, 4018-4030. [DOI], [ADS], [arXiv:astro-ph/0003305] (Cited on page 47.)

Valori, G., Kliem, B., Török, T. and Titov, V. S., 2010, "Testing magnetofrictional extrapolation with the Titov-Démoulin model of solar active regions", Astron. Astrophys., 519, A44. [DOI], [ADS], [arXiv:1005.0254 [astro-ph.SR]] (Cited on page 39.)

van Ballegooijen, A.A. and Martens, P.C.H., 1989, "Formation and eruption of solar prominences", Astrophys. J., 343, 971-984. [DOI], [ADS] (Cited on pages 31 and 32.)

van Ballegooijen, A.A., Priest, E.R. and Mackay, D.H., 2000, "Mean Field Model for the Formation of Filament Channels on the Sun", Astrophys. J., 539, 983-994. [DOI], [ADS] (Cited on page 32.)

van Driel-Gesztelyi, L., Malherbe, J.-M. and Démoulin, P., 2000, "Emergence of a U-loop - subphotospheric link between solar active regions", Astron. Astrophys., 364, 845-852. [ADS] (Cited on page 32.)

Veronig, A.M., Karlický, M., Vršnak, B., Temmer, M., Magdalenić, J., Dennis, B.R., Otruba, W. and Pötzi, W., 2006, "X-ray sources and magnetic reconnection in the X3.9 flare of 2003 November 3", Astron. Astrophys., 446, 675-690. [DOI], [ADS] (Cited on pages 12 and 61.)

Veronig, A.M., Rybák, J., Gömöry, P., Berkebile-Stoiser, S., Temmer, M., Otruba, W., Vršnak, B., Pötzi, W. and Baumgartner, D., 2010, "Multiwavelength Imaging and Spectroscopy of Chromospheric Evaporation in an M-class Solar Flare", Astrophys. J., 719, 655-670. [DOI], [ADS], [arXiv:1007.0930 [astroph.SR]] (Cited on page 63.)

Vršnak, B., 1989, "Soft X-ray emission from a hot turbulent current sheet and the precursor phase of solar flares", Solar Phys., 120, 79-92. [DOI], [ADS] (Cited on page 66.)

Vršnak, B., 1990, "Eruptive instability of cylindrical prominences", Solar Phys., 129, 295-312. [DOI], [ADS] (Cited on page 57.)

Vršnak, B. and Skender, M., 2005, "2 1/2-Dimensional Reconnection Model and Energy Release in Solar Flares", Solar Phys., 226, 97-119. [DOI], [ADS] (Cited on pages 66 and 69.)

Vršnak, B., Temmer, M., Veronig, A., Karlický, M. and Lin, J., 2006, "Shrinking and Cooling of Flare Loops in a Two-Ribbon Flare", Solar Phys., 234, 273-299. [DOI], [ADS] (Cited on pages 12 and 68.) 
Švestka, Z., 1976, Solar Flares, vol. 8 of Geophysics and Astrophysics Monographs, Reidel, Dordrecht; Boston. [ADS] (Cited on pages 5, 10, and 63.)

Švestka, Z. and Cliver, E.W., 1992, "History and Basic Characteristics of Eruptive Flares", in Eruptive Solar Flares, Proceedings of Colloquium No. 133 of the International Astronomical Union, held at Iguazú, Argentina, 2-6 August 1991, (Eds.) Švestka, Z., Jackson, B.V., Machado, M.E., vol. 399 of Lecture Notes in Physics, pp. 1-11, Springer, Berlin; New York. [DOI], [ADS] (Cited on page 8.)

Wang, T., Sui, L. and Qiu, J., 2007, "Direct Observation of High-Speed Plasma Outflows Produced by Magnetic Reconnection in Solar Impulsive Events", Astrophys. J. Lett., 661, L207-L210. [DOI], [ADS], [arXiv:0709.2329] (Cited on page 21.)

Wang, Y.-M., 2000, "EIT Waves and Fast-Mode Propagation in the Solar Corona", Astrophys. J. Lett., 543, L89-L93. [DOI], [ADS] (Cited on page 70.)

Warmuth, A., 2007, "Large-scale Waves and Shocks in the Solar Corona", in The High Energy Solar Corona: Waves, Eruptions, Particles, CESRA Workshop 2004, (Eds.) Klein, K.-L., MacKinnon, A.L., vol. 725 of Lecture Notes in Physics, pp. 107-138, Springer, Berlin; New York. [DOI], [ADS] (Cited on page 70.)

Warmuth, A., Vršnak, B., Aurass, H. and Hanslmeier, A., 2001, "Evolution of Two EIT/H $\alpha$ Moreton Waves", Astrophys. J. Lett., 560, L105-L109. [DOI], [ADS] (Cited on page 70.)

Warren, H.P., Winebarger, A.R. and Mariska, J.T., 2003, "Evolving Active Region Loops Observed with the Transition Region and Coronal explorer. II. Time-dependent Hydrodynamic Simulations", Astrophys. J., 593, 1174-1186. [DOI], [ADS] (Cited on page 69.)

Watanabe, K., Gros, M., Stoker, P.H., Kudela, K., Lopate, C., Valdés-Galicia, J.F., Hurtado, A., Musalem, O., Ogasawara, R., Mizumoto, Y., Nakagiri, M., Miyashita, A., Matsubara, Y., Sako, T., Muraki, Y., Sakai, T. and Shibata, S., 2006, "Solar Neutron Events of 2003 October-November", Astrophys. J., 636, 1135-1144. [DOI], [ADS], [arXiv:astro-ph/0509527] (Cited on page 71.)

Watanabe, T., 1994, "Characteristics of Microflares Seen in Helium-Like Sulphur Spectra: GOES A-Class Flares During the Minimum Activity Phase", in Proceedings of Kofu Symposium 'New Look at the Sun with Emphasis on Advanced Observations of Coronal Dynamics and Flares', Kofu, Japan, September 6-10, 1993, (Eds.) Enome, S., Hirayama, T., vol. 360 of NRO Report, pp. 99-103, Nobeyama Radio Observatory, Nobeyama. [ADS]. Online version (accessed 10 December 2009):

http://solar.nro.nao.ac.jp/meeting/kofu/ (Cited on page 17.)

Watanabe, T., Kozuka, Y., Ohyama, M., Kojima, M., Yamaguchi, K., Watari, S.-I., Tsuneta, S., Joselyn, J.A., Harvey, K.L., Acton, L.W. and Klimchuk, J.A., 1992, "Coronal/interplanetary disturbances associated with disappearing solar filaments", Publ. Astron. Soc. Japan, 44, L199-L204. [ADS] (Cited on page 14.)

Wheatland, M.S., Sturrock, P.A. and Roumeliotis, G., 2000, "An Optimization Approach to Reconstructing Force-free Fields", Astrophys. J., 540, 1150-1155. [DOI], [ADS] (Cited on page 39.)

Wiegelmann, T., 2008, "Nonlinear force-free modeling of the solar coronal magnetic field", J. Geophys. Res., 113(A12), 3. [DOI], [ADS], [arXiv:0801.2902] (Cited on page 39.)

Wiegelmann, T. and Neukirch, T., 2006, "An optimization principle for the computation of MHD equilibria in the solar corona", Astron. Astrophys., 457, 1053-1058. [DOI], [ADS], [arXiv:astro-ph/0612625] (Cited on page 39.)

Wiegelmann, T., Schindler, K. and Neukirch, T., 2000, "Helmet Streamers with Triple Structures: Simulations of resistive dynamics", Solar Phys., 191, 391-407. [ADS], [arXiv:0801.3187] (Cited on page 39.)

Wu, S.T., Guo, W.P. and Dryer, M., 1997, "Dynamical Evolution of a Coronal Streamer-Flux Rope System. II. A Self-Consistent Non-Planar Magnetohydrodynamic Simulation", Solar Phys., 170, 265-282. [ADS] (Cited on page 57.) 
Wu, S.T., Zheng, H., Wang, S., Thompson, B.J., Plunkett, S.P., Zhao, X.P. and Dryer, M., 2001, "Threedimensional numerical simulation of MHD waves observed by the Extreme Ultraviolet Imaging Telescope", J. Geophys. Res., 106, 25,089-25,102. [DOI], [ADS] (Cited on page 70.)

Yamada, M., Ji, H., Hsu, S., Carter, T., Kulsrud, R. and Trintchouk, F., 2000, "Experimental investigation of the neutral sheet profile during magnetic reconnection", Phys. Plasmas, 7, 1781-1787. [DOI], [ADS] (Cited on page 49.)

Yang, G., Xu, Y., Cao, W., Wang, H., Denker, C. and Rimmele, T.R., 2004, "Photospheric Shear Flows along the Magnetic Neutral Line of Active Region 10486 prior to an X10 Flare", Astrophys. J. Lett., 617, L151-L154. [DOI], [ADS] (Cited on page 44.)

Yang, W.H., Sturrock, P.A. and Antiochos, S.K., 1986, "Force-free magnetic fields: The magneto-frictional method", Astrophys. J., 309, 383-391. [DOI], [ADS] (Cited on page 39.)

Yashiro, S., Gopalswamy, N., Michalek, G., St Cyr, O.C., Plunkett, S.P., Rich, N.B. and Howard, R.A., 2004, "A catalog of white light coronal mass ejections observed by the SOHO spacecraft", J. Geophys. Res., 109, A07105. [DOI], [ADS] (Cited on page 10.)

Yokoyama, T. and Shibata, K., 1994, "What is the condition for fast magnetic reconnection?", Astrophys. J. Lett., 436, L197-L200. [DOI], [ADS] (Cited on pages 47 and 50.)

Yokoyama, T. and Shibata, K., 1995, "Magnetic reconnection as the origin of X-ray jets and H $\alpha$ surges on the Sun", Nature, 375, 42-44. [DOI], [ADS] (Cited on page 50.)

Yokoyama, T. and Shibata, K., 1996, "Numerical Simulation of Solar Coronal X-Ray Jets Based on the Magnetic Reconnection Model", Publ. Astron. Soc. Japan, 48, 353-376. [ADS] (Cited on pages 19, 25, 50, and 51.)

Yokoyama, T. and Shibata, K., 1997, "Magnetic Reconnection Coupled with Heat Conduction", Astrophys. J. Lett., 474, L61. [DOI], [ADS] (Cited on pages 8, 66, and 67.)

Yokoyama, T. and Shibata, K., 1998, "A Two-dimensional Magnetohydrodynamic Simulation of Chromospheric Evaporation in a Solar Flare Based on a Magnetic Reconnection Model", Astrophys. J. Lett., 494, L113. [DOI], [ADS] (Cited on pages 8, 66, 67, 68, and 69.)

Yokoyama, T. and Shibata, K., 2001, "Magnetohydrodynamic Simulation of a Solar Flare with Chromospheric Evaporation Effect Based on the Magnetic Reconnection Model", Astrophys. J., 549, 1160-1174. [DOI], [ADS] (Cited on pages 8 and 68.)

Yokoyama, T., Akita, K., Morimoto, T., Inoue, K. and Newmark, J., 2001, "Clear Evidence of Reconnection Inflow of a Solar Flare", Astrophys. J. Lett., 546, L69-L72. [DOI], [ADS] (Cited on pages 21 and 46.)

Zhang, M. and Low, B.C., 2003, "Magnetic Flux Emergence into the Solar Corona. III. The Role of Magnetic Helicity Conservation", Astrophys. J., 584, 479-496. [DOI], [ADS] (Cited on page 10.)

Zirin, H., 1974, "Solar flares", Vistas Astron., 16, 1-34. [DOI], [ADS] (Cited on page 9.)

Zirin, H., 1988, Astrophysics of the Sun, Cambridge University Press, Cambridge; New York. [ADS] (Cited on pages 5 and 10.)

Zwaan, C., 1985, "The emergence of magnetic flux", Solar Phys., 100, 397-414. [DOI], [ADS] (Cited on pages 8,41 , and 43.$)$ 